\title{
SELECTING A MIX OF DISPATCHING RULES \\ FOR A JOB SHOP BY USING ARTIFICIAL \\ NEURAL NETWORKS
}

\author{
DEC - 92004
}

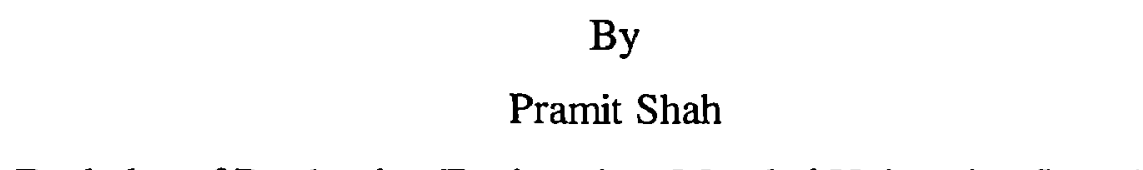

Bachelor of Production Engineering, Mumbai University, June 1997

A thesis presented to the Ryerson University

in fulfillment of the

thesis requirement for the degree of

Master of Applied Science

in

Mechanical and Industrial Engineering

PROPERTY OF
Ryerson University Librory

Toronto,Ontario,Canada, 2004

@ (Publisher Pramit Shah) 2004 


\section{UMI Number: EC52985}

\section{All rights reserved \\ INFORMATION TO USERS}

The quality of this reproduction is dependent upon the quality of the copy submitted. Broken or indistinct print, colored or poor quality illustrations and photographs, print bleed-through, substandard margins, and improper alignment can adversely affect reproduction.

In the unlikely event that the author did not send a complete manuscript and there are missing pages, these will be noted. Also, if unauthorized copyright material had to be removed, a note will indicate the deletion.

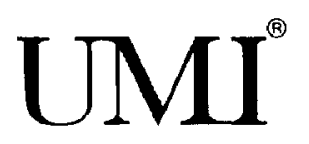

UMI Microform EC52985

Copyright 2008 by ProQuest LLC

All rights reserved. This microform edition is protected against unauthorized copying under Title 17, United States Code.

ProQuest LLC

789 East Eisenhower Parkway

P.O. Box 1346

Ann Arbor, MI 48106-1346 


\section{AUTHOR'S DECLARATION}

I hereby declare that I am the sole author of this thesis.

I authorize the Ryerson University to lend this thesis to other institutions or individuals for the purpose of scholarly research.

Pramit Shah

I further authorize the Ryerson University to reproduce this thesis by photocopying or by other means, in total or in part, at the request of other institutions or individuals for the purpose of scholarly research.

Pramit Shah

(ii) 
Ryerson University requires the signature of all persons using or photocopying this thesis. Please sign below, and give address and date.

\begin{tabular}{|c|c|c|}
\hline Name & Address & Date \\
\hline & & \\
\hline & & \\
\hline & & \\
\hline & & \\
\hline & & \\
\hline & & \\
\hline & & \\
\hline & & \\
\hline & & \\
\hline & & \\
\hline & & \\
\hline & & \\
\hline & & \\
\hline & & \\
\hline & & \\
\hline & & \\
\hline & & \\
\hline & & \\
\hline & & \\
\hline & & \\
\hline & & \\
\hline
\end{tabular}




\section{ABSTRACT \\ Selecting a Mix of Dispatching Rules For a Job Shop by Using

\author{
Artificial Neural Networks \\ @ Publisher Pramit Shah, 2004 \\ Master of Applied Science \\ In the program of Mechanical and Industrial Engineering \\ Ryerson University
}

Dispatching rules are a popular and commonly researched technique for scheduling tasks in job shops. Much of the past research has looked at the performance of various dispatching rules when a single rule is applied in common on all machines However, better schedules can frequently be obtained if the machines are allowed to use different rules from one another. This research investigates an intelligent system that selects dispatching rules to use on each machine in the shop, based on a statistical description of the routings, processing times and mix of the jobs to be processed. Randomly generated problems are scheduled using permutations of three different dispatching rules on five machines. A neural network is then trained by using a commercial package to associate the statistical description of each problem with its best solution. Once trained, a network is able to recommend for new problems a dispatching rule to use on each machine. Two networks were trained separately for minimizing makespan and the total flowtime in the job shop. Test results showed that the combination of dispatching rules suggested by the trained networks produced better results for both objectives than the alternative of using the one identical rule on all machines. 


\section{ACKNOWLEDGEMENTS}

I would like to express my gratitude to all those who contributed to this work directly as well as indirectly.

To work with Dr. Ahmed El-bouri was a lifetime opportunity. His trust in his students, continuous encouragement, strong support, insightful guidance makes a student perform far better than he is. I sincerely wish to thank him for all the help provided to me. I really enjoyed the exchange of ideas that helped me to formulate this work. Thank you. Sir!

The financial support through Professor Ahmed El-bouri of Department of Mechanical Engineering- Ryerson University is greatly appreciated.

My family had an indirect contribution to this research. Especially I would like to thank my wife, Miloni for her patience, understanding and emotional support. Thank you from the bottom of my heart!

I am also thankful to all my fellow graduate students specially Akram and Jayesh, for keeping my spirit up.

And above all, I thank God for giving me the strength and determination. 
To my parents and Miloni 
ABSTRACT-

$A C K N O W L E D G E M E N T S$

TABLE OF CONTENTS-

LIST OF FIGURES-

LIST OF TABLES

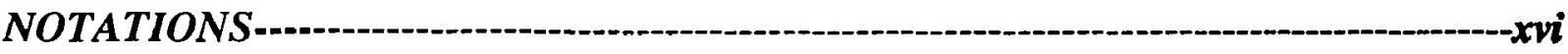

GLOSSARY -

Chapter 1: Introduction and Background

1.1 Introduction--

1.2 Problem Definition--

1.3 Dispatching Rules--

1.4 Thesis Outline--

Chapter 2: Literature Review

2.1 Literature Review---10

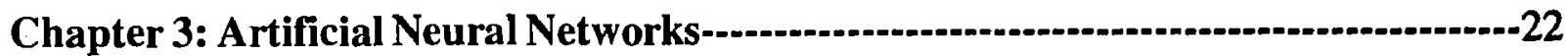

3.1 Introduction to Artificial Neural Networks (ANN)---_-

3.1.1 Basic Concepts-- 
3.1.3 Classification of Neural Network Architectures ---_-_-_---25

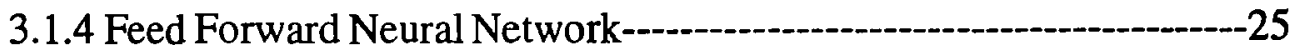

3.1.5 Learning rule--1---

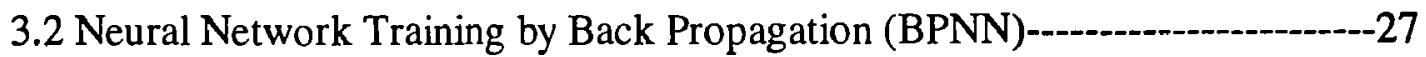

3.2.1 Scaling Function-

3.2.2 Transfer Function--

3.2.3 Delta Rule--

3.2.4 Type of Datasets---_-

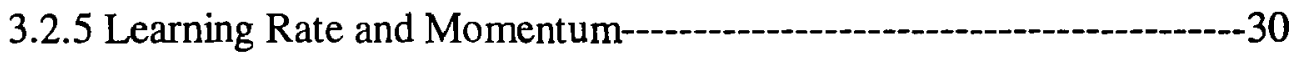

3.2.6 Initial Weights--

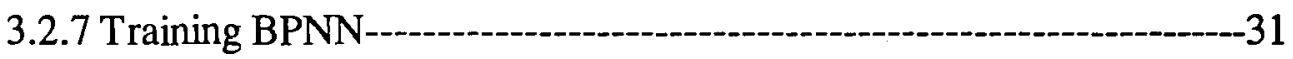

Chapter 4: Methodology and Design of BPNN-33

4.1 Design of a BPNN for Job Shop Scheduling

4.2 Problem Analysis--

4.3 Data Acquisition and Preparation -

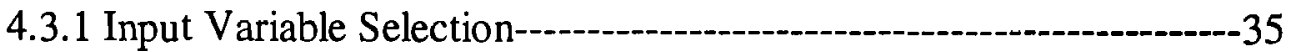

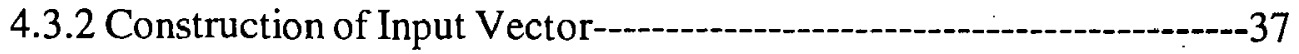

4.3.3 Output Layer--

4.3.4 Data Collection--

4.3.5 Validation of Data Set Output--

4.3.6 Data Sorting - 
4.4.1 Hidden Layer-

4.4.2 Hidden Units (Neurons)---

4.4.3 Training Stopping Criteria--

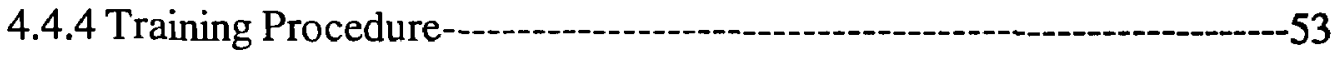

4.4.5 To Find Optimal Number of Hidden Neurons (Units)--_-_-_-_-_--53

4.5 Training BPNN-

4.6 Implementation-----

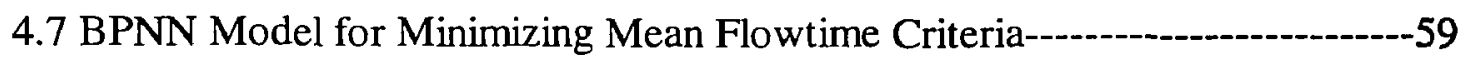

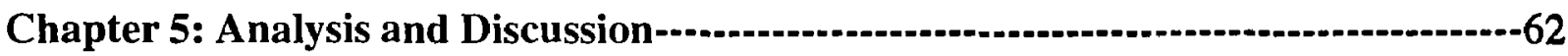

5.1 Analysis of Trained Network-

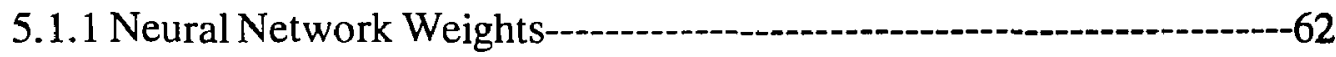

5.2 Test Problems -

5.3 Test results for Minimizing Makespan --.--on

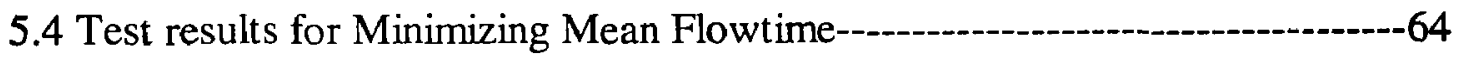

5.5 Further Discussion on Results --

5.6 Analysis of Variance (ANOVA) -

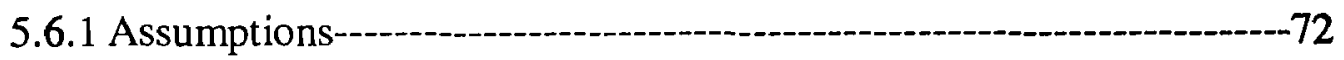

5.6.2 Data Representation--

5.6.3 General Logic - Analysis of Variance--

5.6.4 ANOVA Calculations -

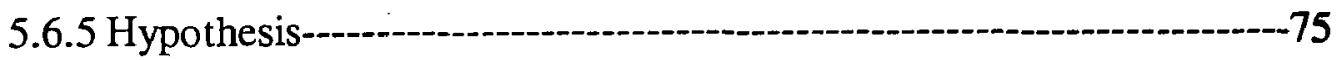


5.7 ANOVA Results for Makespan

5.8 ANOVA Results for Mean Flowtime

Chapter 6: Conclusion and Future research

6.1 Summary $-80$

6.2 Conclusions$-81$

6.3 Future Research

References $-84$

\section{Appendix A: C++ Programs}

Appendix A1: Problem Generation-

Appendix A2: Training Set Generation A3

Appendix A3: Comparison of BPPNN Results with Other Dispatching Rule

Results A13

Appendix A4: Test Problem Generation

Appendix B: Arena Simulation Model$-\mathrm{B} 1$

\section{Appendix C: Experimental Setup}

Appendix C1: Determining Number of Hidden Neurons for Makespan-----------_1 Appendix C2: Determining Number of Hidden Neurons for Mean Flowtime ---C2 


\section{Appendix D: Neural Weights}

Appendix D1: Makespan Network-

Appendix D2: Mean Flowtime Network-10

\section{Appendix E: Comparison Results}

Appendix E1: Comparison of BPNN with makespans from optimal rule

combinations for $\mathrm{n}=10$ to 100 -.-.---E1

Appendix E2: Comparison of BPNN with mean flowtimes from optimal rule

combinations for $\mathrm{n}=10$ to 100 


\section{LIST OF FIGURES}

Figure 1.1 Schematic layout of a job shop, illustrating the routes followed by two different jobs $-2$

Figure 2.1 Degree of artificial intelligence required by different scheduling approaches $-15$

Figure 3.1 Basic features of a single biological neuron ------

Figure 3.2 Processing elements --

Figure 3.3 Classification of common neural network architectures --------------------------25

Figure 3.4 Multi-layer feed forward neural network ---

Figure 4.1 Design methodology for building ANN-

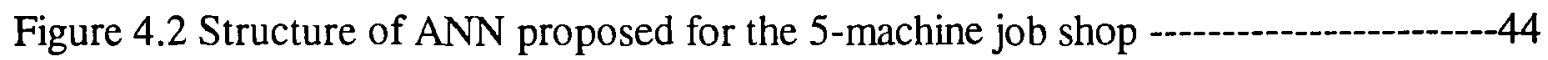

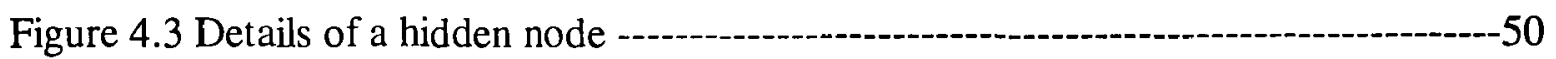

Figure 4.4 Effect of number of hidden neurons on the minimum average error for

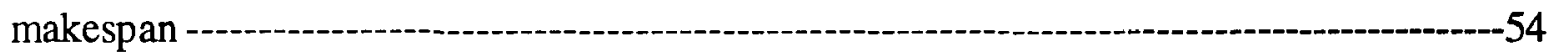

Figure 4.5 BPNN generalization vs. minimum average error for makespan----------------57

Figure 4.6 Effect of number of hidden neurons on the minimum average error for mean flowtime $-60$

Figure 5.1 BPNN makespans compared to makespans from optimal rule combinations for $\mathrm{n}=50$

Figure 5.2 BPNN mean flowtime compared to mean flowtime from optimal rule combinations for $n=50$

Figure 5.3 Comparison of BPNN, SPT, LPT and MWKR with respect to makespan of optimal rule combinations 
Figure 5.4 Comparison of BPNN, SPT, WINQ+PT and LWKR with respect to mean flowtime of optimal rule combinations -.-.-1 


\section{LIST OF TABLES}

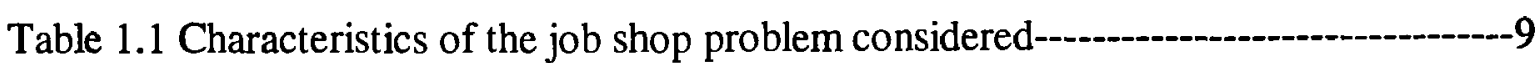

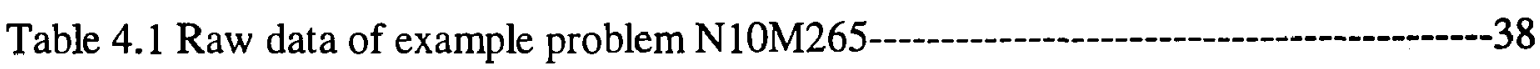

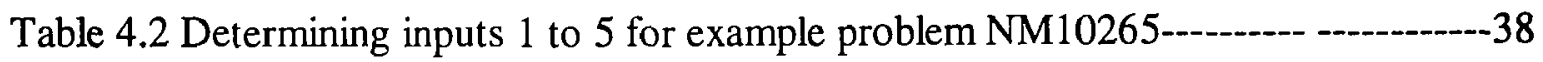

Table 4.3 Inputs 6 to 10 for example problem N10M265---

Table 4.4 Example of normalized value for inputs 11 through 15 for problem

N10M265--10-1-

Table 4.5 15-unit input vector representing job shop problem of Table 4.1---------------40

Table 4.6 Optimal rule combinations for example problem N10M265---:------------------42

Table 4.7 Numerical representation for optimal rule combinations of Table 4.6----------43

Table 4.8 15-unit vector representing desired output for example problem

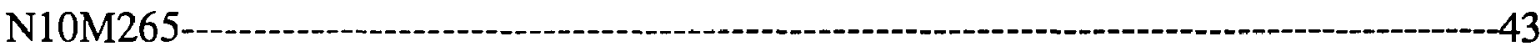

Table 4.9 Sample of input and output pattern pairs --

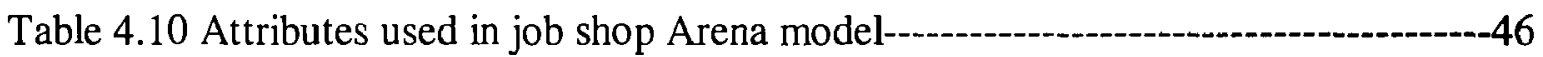

Table 4.11 Input and output patterns of Table 4.9 after sorting ---

Table 4.12 Total makespans using BPNN with various number of hidden neurons ------54

Table 4.13 Training and generalization results for minimizing makespan -----------------56

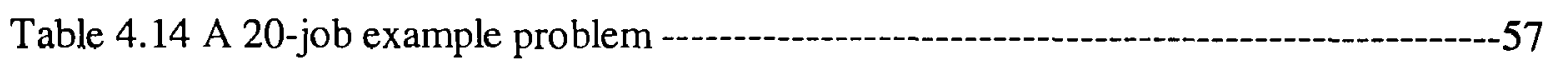

Table 4.15 Input vector for example problem of Table 4.14

Table 4.16 BPNN output for example problem of Table 4.14

Table 4.17 Total mean flowtimes for BPNN with various numbers of hidden neurons 
Table 4.18 Mean flowtime BPNN output for example problem of Table 4.14-------------60

Table 5.1 Summary of total makespan in sets of 50 test problems for various n ---a---64

Table 5.2 Results for individual problems for $n=50$ (set no. 9 in Table 5.1) -

Table 5.3 Summary of total mean flowtime results for test sets consisting of 50

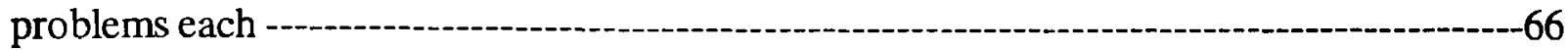

Table 5.4 Mean flowtime for individual test problems for $n=50$

(set no. 9 in Table 5.3) -

Table 5.5 Experimental data for ANOVA (average makespan)---

Table 5.6 ANOVA results for the performance objective of minimizing makespan -----76

Table 5.7 LSD calculations for the minimization of makespan experiment ----------------77

Table 5.8 ANOVA experimental data (average mean flowtime) ----o---o---o-----------78

Table 5.9 ANOVA results for the performance objective of minimizing mean flowtime -

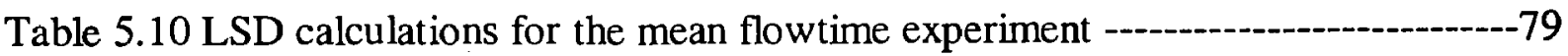




\section{NOTATIONS}

\begin{tabular}{|c|c|}
\hline$m$ & Number of machines \\
\hline$i$ & Subscript for jobs, $\mathrm{i}=1,2,3, \ldots \ldots$. \\
\hline$j$ & Subscript for operation, $\mathrm{j}=1,2,3, \ldots . \mathrm{m}$. \\
\hline$k$ & Subscript for machines, $\mathrm{k}=1,2,3 \ldots . \mathrm{m}$. \\
\hline$\lambda_{i}$ & Time of arrival of job on the shop floor. \\
\hline$P_{i j k}$ & Processing time of operation $\mathrm{j}$ of job $\mathrm{i}$ on machine $\mathbf{k}$. \\
\hline$Q_{i k}$ & Number of operations $\mathrm{j}$ required to complete job $\mathrm{i}$ \\
\hline$n_{i}$ & Number of input neurons. \\
\hline$n_{0}$ & Number of output neurons. \\
\hline$R_{k}$ & Mean routing order on machine $\mathbf{k}$. \\
\hline$C_{i}$ & Completion time of job $\mathrm{i}$. \\
\hline$C_{\max }$ & Maximum completion time or makespan. \\
\hline$F_{i}$ & Flow time of job $i$. \\
\hline$\sigma$ & Standard deviation. \\
\hline $\operatorname{Var}(\mathrm{X})$ & Variance \\
\hline$K$ & Number of treatments for ANOVA experiments. \\
\hline$X$. & Grand mean. \\
\hline$X$ & Group mean. \\
\hline
\end{tabular}




\section{GLOSSARY}

Dispatching rules

Rules based on the smallest processing time or least work remaining

LWKR: - Select the job with the least total work remaining work

SPT:- Select the job with the least intermediate processing time

TPT: - Select the job with least total processing time

Rules based on the largest processing time or most work content

LPT:- Select the job with the most intermediate processing time

LOPNR: - Select the job whose least number of operations remaining

MOPNR: - Select the job whose most number of operations remaining.

MWKR:- Select the job with the most total work remaining work

MWKR-P:- Jobs ranked by MWKR after the present scheduling operation

MWKR/P: - Jobs ranked by the greatest ratio of total remaining work to processing time of schedulable operation

\section{Rules based on due date}

DS: - Select the job with the smallest slack, where slack is the due date less the total work remaining. This is also called as the dynamic slack.

DDT / EDD: - Jobs ranked according to their earliest due date

SLK/RO: - Select the job wit the smallest ratio of its dynamic slack to the number of remaining operations

Rule based on arrival

AT: - Select the job based on earliest arrival time 
FIFO: - First in first out

LIFO: - Last in first out

RAN: - Select the job randomly

Rule based on queue status

WINQ: - Select the job based whose workload in the next queue is least

NINQ: - Select the job based on the number of jobs waiting in the queue of the next machine. 



\section{CHAPTER 1}

\section{Introduction and Background}

\subsection{Introduction}

In real world applications, one of the most important aspects of competitiveness and success of an organization is efficient production management and timely decisions. Such decisions are often constrained by specific objectives and requirements. These constraints are frequently incompatible in nature, and as the number of such constraints increases, the decision process becomes more complex. Scheduling decisions are an important component of overall operational control in any manufacturing system.

The decision-making requirements for scheduling production systems have been a fascinating research topic for more than three decades. The goal of scheduling is to ensure that production objectives are met optimally. Researchers have developed numerous models and methodologies for the planning and scheduling control of production activities for different manufacturing layouts. Two of the most researched models are flow shop and job shop.

A flow shop is a model in which machines are arranged in series. In this system, jobs flow from an initial machine (resource), through several intermediate machines, and ultimately to a final machine. A job may skip a particular machine, but all jobs must be processed according to a fixed route. The job shop differs from the flow shop in one important aspect: the flow of work is not unidirectional, meaning that jobs need not visit the machines in the same order. The problem in the job shop is to schedule $n$ jobs on $m$ 
machines such that one or more performance objectives is optimized. Each job requires $j$ number of operations (tasks) that are to be processed on these machines. Generally, a job may not require all the machines and it may visit the same machine more than once.

A scheduling problem in a job shop is defined by several attributes, namely, the number of jobs; the number of machines; the processing order of the jobs on the machines (or routing); processing time of each job on different machines; job arrival times, and last but not the least, the performance objective to be achieved. Figure 1.1 displays a general job shop model. It illustrates the routes taken by two different jobs through the shop. In this instance both the jobs visit machine number three after completion of two operations as per their respective job routes. Supposing Job 1 completes both its operations prior to job 2 , then job 2 may have to wait in the machine buffer (or queue) if the machine is still busy with job 1.

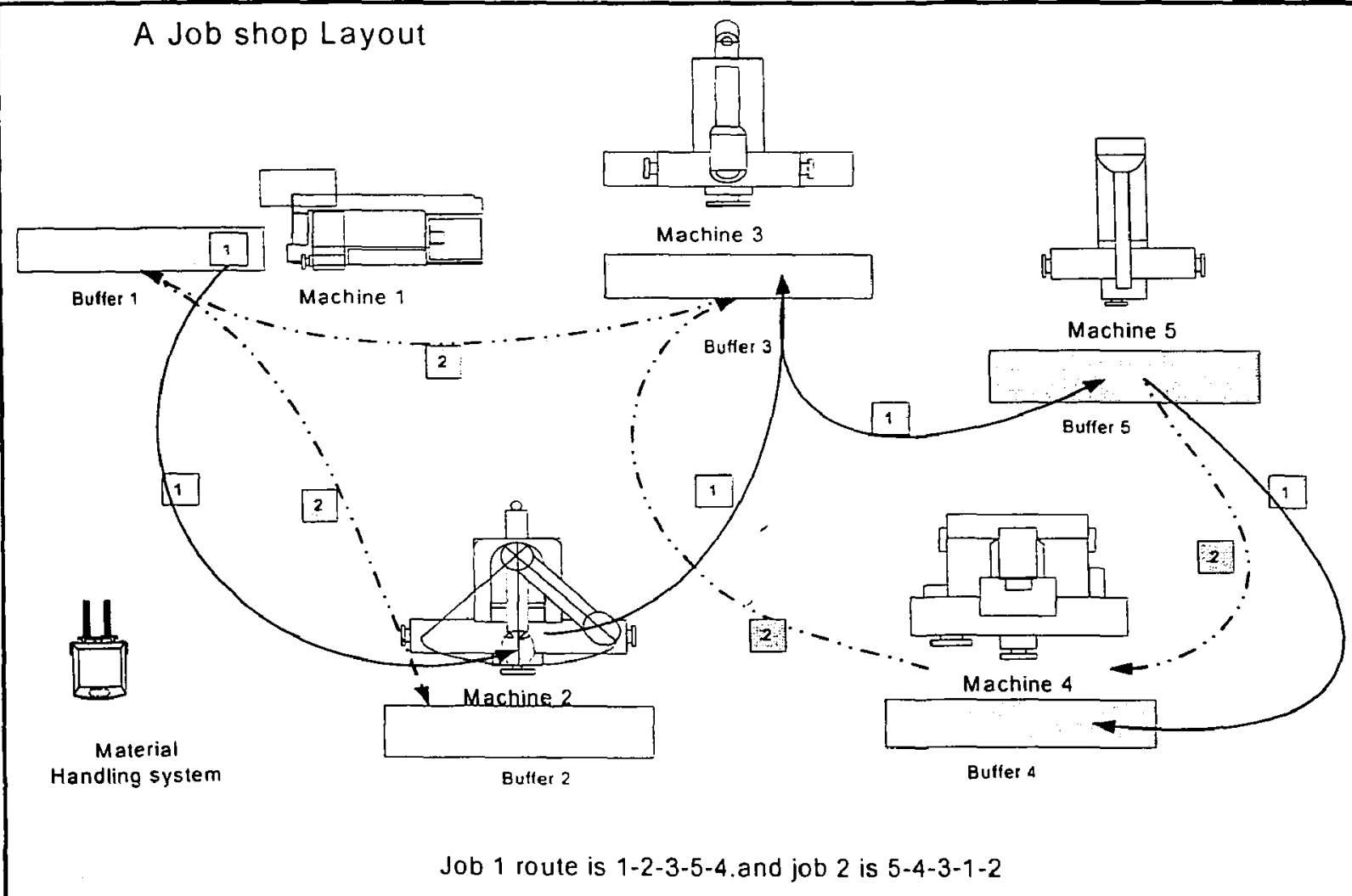

Fig 1.1 Schematic layout of a job shop, illustrating the routes followed by two different jobs. 
For scheduling $\mathrm{n}$ jobs on a single machine there are $\mathrm{n}$ ! possible schedules, but in the case of a job shop the number of possible combinations is $(n !)^{m}$, when each job has one and only one operation on each machine (Conway, 1967). For example with 10 jobs on 5 machines there are $6.92 \times 10^{32}$ possible number of schedules. Thus, job shop scheduling is a NP-hard problem (Pinedo, 1995) and, consequently, it is not practical to search for a global optimal solution other than for very small problems.

A general job shop problem can be either static or dynamic. The static job shop assumes that all jobs are available at the beginning of the planning phase. On the other hand, the dynamic job shop problem assumes that the arrival times of the jobs are unknown, and that jobs arrive at random intervals throughout the production cycle. A schedule can be evaluated by its ability to meet production objectives like minimizing the make span (or completion time), the mean flow time and mean tardiness. A schedule that results in minimum completion time for a particular problem does not necessarily minimize the mean flowtime in that problem. Hence, the aim of the scheduling is to find a schedule for processing all jobs, such that one or more specified performance measures are optimized.

A number of job shop scheduling techniques have been developed for solving static and dynamic problems. Among these are the dispatching rules, which are a popular and commonly researched technique for scheduling tasks in a job shop. A dispatching rule (DR) is used to select the next job to be processed from a set of jobs waiting in the queue (or buffer) when a machine becomes free. The simplicity and ease in application of dispatching rules (DRs) have made them a practical tool for scheduling in the real world. However, there are some shortcomings in the use of dispatching rules. First, none of the dispatching rules dominates the others for the important performance criteria like mean 
flow time, mean tardiness, etc. This implies that DRs are problem dependent, and one DR that gives a good result for one problem may not give an equally good result in another. Due to the dynamic and changing characteristics of the jobs as they are processed, a job shop may be able to meet the performance objectives better by judiciously changing the DR on individual machines over time, or by using a combination of different rules for the machines.

As a result of the scheduling complexity in modern manufacturing systems, Artificial Intelligence (AI) techniques have been considered as a scheduling decision tool; one technique that has shown promise is the Artificial Neural Network (ANN). In simple terms, Artificial Neural Networks apply knowledge gained from past experience to new problems or situations. An ANN looks for patterns in "training" sets of data, learns these patterns and develops the ability to correctly classify new patterns. This approach in a job shop that uses dispatching rules requires the neural network to be prepared by selecting a set of training examples for different performance measures, and finding from simulation (or other) studies the optimal rules to use in these examples. Information from these optimal solutions is then used to "teach" the network to select the most appropriate dispatching rule from several candidate / rules. Once this procedure is completed, the trained neural network is capable of providing faster solutions to new problems which were previously unseen by the network.

This research addresses the problem of an appropriate selection of dispatching rules (in this case from three alternatives) to use for each of the machines in a job shop by using artificial neural networks. Two separate artificial neural networks are developed for the performance criteria of minimizing makespan and minimizing mean flowtime. Each of these criteria have a different set of competing DRs to choose from. 


\subsection{Problem Definition}

A job shop problem of interest is a 5-machine job shop. In actual manufacturing systems, particularly those that follow a group technology concept, the number of machines used can be reasonably expected to be between 3 to 10. Also, it seems to be a consensus among researchers that a four-machine job shop is adequate to represent the complexity involved in a large job shop (Kiran et al, 1984). Thus, the number of machines chosen for this study is 5 machines, with number of jobs ranging from 10 jobs to a maximum of 100 .

In this study, a static job shop is considered with arrival time $\lambda_{i}=0$ for all the jobs. Thus, all the jobs that are to be processed are available at the start of the scheduling period. It has been assumed that all the jobs have two sets of attributes. The first one is the work flow $Q_{i k}$ pattern (or route), that is machine $k$ 's order in job i's route. At the beginning of the schedule, each job's flow pattern is defined with the condition that each job visits all machines once. In other words, each job has a specific precedence order of operations, which has to be followed before exiting from the system. For example, a specific job $i$ may have the precedence order $4 \longrightarrow 5 \longrightarrow 3 \longrightarrow 2 \longrightarrow 1$, indicating that

job $i$ 's first operation must be done on machine 4 , its second operation on machine 5 , and so on.

The second characteristic is the processing time $\left(P_{i j k}\right)$ representing the $j^{\text {th }}$ operation of job $i$ on machine $\mathrm{k}$. The time needed by each operation $j$ of job $i$ on machine $k$ is known in advance. In this study, deterministic processing times for these operations are generated from the discrete uniform distribution $U(10,99)$ integer time units. For 
example, a specific job on 5 machines may have processing times of 45-95-61-20-35 corresponding to the machines on the job's route.

Once operation $j$ of job $i$ has been completed, it will be transferred with the help of a material handling system to the next machine, as per the job's route for the next operation $j+1$ if that machine is free, or to a buffer for that machine otherwise. It has been assumed that the transfer time between machines is negligible and that the material handling system is always available whenever required. Hence, jobs are either in process on a machine, or waiting in a buffer for processing.

"Schedules are generally evaluated by aggregate quantities that involve information about all jobs, resulting in one-dimensional performance measures" (Baker, 2002). The following two performance measures or criteria or objectives are considered in this study.

a) Makespan: The makespan measures the total time taken by a given schedule to complete the set of available jobs. In other words, it is the time at which the last job exits from the shop. This measure is defined, for a sequence of $\mathrm{n}$ jobs, by

$$
\mathrm{C}_{\max }=\max _{\mathrm{i}}\left\{C_{i}\right\}
$$

Where $C_{i}=$ Completion time of job $i=1,2,3 \ldots \mathrm{n}$

The objective of scheduling the jobs in a way that minimizes the makespan is an important one, because it reduces throughput time for processing a batch of different jobs. b) Mean Flowtime: Flowtime represents the total time spent in a job shop. This includes actual machine processing time plus time spent waiting in buffers.

The mean flowtime is given by:

$$
\bar{F}=\frac{1}{n} \sum_{i=1}^{n} F_{i}
$$


Where $F_{i}=$ Flow time or the time spent by job $i$ in the system

The objective of minimizing the mean flowtime is a common objective in scheduling, because it acts to reduce work-in process and inventory levels.

The job shop model considered in this study is based on the following explicit assumptions:

- Jobs are independent and consist of strictly ordered operational sequences (or job routes). Furthermore, all jobs have equal weights (importance).

- Job pre-emption or cancellation is not allowed. Once the processing of any operation has started on a machine, it cannot be interrupted before its completion, and then resumed at a later time.

- There is only one machine of each type in the shop, and operations for two different jobs cannot be processed simultaneously on the same machine.

- Set up time is negligible.

- An operation may not begin until its predecessor is complete.

- Each machine is continuously available for production; machine breakdown or downtime is not considered.

- There are no alternate routes for any job.

- The buffer capacity is unlimited and machine blocking does not occur.

\subsection{Dispatching Rules (DRs)}

Dispatching rules or (priority rules) refer to the procedure used to prioritize the jobs that are waiting in queue for a machine. The dispatching rule therefore picks the next job 
to load on the resources or machine. There are more than 100 dispatching rules surveyed by Panwalkar and Iskander, (1977). Dispatching rules use job specific information such as processing time, due date, remaining number of operations, etc. The rules considered in this study are:

\section{a) Rules based on shortest processing time}

- Shortest processing time (SPT): - Priority is given to the job with the shortest immediate processing time (i.e., smallest processing time on the current resource).

- Least work remaining (LWKR): - Priority is given to the job with the least sum of remaining operation processing times.

\section{b) Rules based on Longest processing time}

- Longest processing time (LPT): - Priority is given to the job with the longest immediate processing time (i.e., largest processing time on the current resource).

- Most work remaining (MWKR) - Priority is given to the job with the total work remaining processing times.

c) Rule based on Queue status

- Work in the next queue (WINQ + PT): - Priority is given to the job with the least workload in the next queue it will visit, plus the processing time $P_{i j k}$ on the current resource.

The Following Table1.1 summarizes the job shop problem parameters considered in this research. 


\begin{tabular}{|c|c|}
\hline Number of jobs & From 10 to l00 \\
\hline Number of machines & 5 \\
\hline Job arrival times & All jobs are available at the start of schedule \\
\hline Flow pattem & Process routes \\
\hline Processing time & Deterministic integers drawn from U (10,99) \\
\hline Performance measures & 1) Minimizing makespan \\
\hline Dispatching rules & 2) Minimizing mean flowtime \\
\hline Job shop scheduling technique & SPT, LPT, MWKR, LWKR, WINQ+PT \\
& \\
\hline
\end{tabular}

Table 1.1 Characteristic of the job shop problem under consideration.

\subsection{Thesis outline}

The rest of the thesis is organized as follows: Chapter 2 provides a detailed literature review on the dispatching rules, neural networks for scheduling, and past research in selecting combinations of dispatching rules by different techniques. Chapter 3 presents a background introduction to neural networks. Chapter 4 provides a step-by-step guideline on building a feed forward back-propagated supervised neural network for selecting dispatching rule combinations. Chapter 5 analyses the output generated from the trained network and examines the generalization capability of the trained network. Chapter 6 provides a summary and a conclusion, and discusses further research scope. 


\section{CHAPTER 2}

\section{Literature Review}

\subsection{Literature Review}

Scheduling jobs is an important aspect of a job shop manufacturing system environment, for it can have a deep impact over the system's performance efficiency. Job shop scheduling has been studied extensively over the last three decades. Many approaches have been developed to solve the static job shop scheduling problem, and some of the well-known approaches can be found in French, (1982) and Pinedo, (1995).

However, for a practical application, scheduling decisions are usually taken in real time considering existing constraints. Some of such constraints are the state of the shop floor (e.g., availability of resources), characteristics of the production program (e.g., part routing, due date of jobs) and production objectives (e.g., minimizing makespan) to be achieved. Nevertheless, some of the uncertain variables such as breakdown / failures of resources, new jobs prompted during a production cycle, etc. also need to be considered. For these reasons many researchers tend to approach the job shop scheduling issue through the acceptance of heuristic dispatching rules rather than seeking a deterministic optimal solution to the problem.

This literature review reports on various job shop scheduling approaches. Both Jones and Rabelo, (1996) and Blazewicz et al, (1996) have done detailed surveys on various job shop scheduling techniques, which can be categorized mainly as either exact or approximation methods. 
The category of exact methods includes all the mathematical models. These guarantee optimal solutions for a job shop problem. However, their application is limited 10 a smaller numbers of jobs and resources. This is due to some of the limitations like computational requirements for obtaining an optimal solution, difficulty in formulation of material flow constraints, etc. Also. the development in computational power of the computer has sharply improved the use of such approaches, but nevertheless its utilization remains limited.

Branch and bound is one such mathematical technique that deals with NP- hardness of scheduling problems by decomposition into smaller sub problems that may be solved for optimality. Blazewicz et al, (1996) presented a detailed discussion on the success and the limitations of this method in job shop problems.

The category of approximation methods includes numerous algorithms and techniques that are developed for producing good solutions, which can be reasonably close to optimal results. These techniques can be further categorized as dispatching / priority rules, Artificial lntelligence (AI) techniques and other heuristic methods. They are used either to obtain a best sequence of jobs for the desired performance objective, or to select from various dispatching rules ones to apply on the machines, based on the current or prevailing conditions.

One of the most common approaches to dynamically schedule jobs is to use dispatching rules (DRs). These rules are sometimes called scheduling rules, or priority rules. They are defined by Blackstone et al, (1982) as a "Rule used to select the next job to process from jobs awaiting service." Dispatching rules are widely used in practice and a considerable body of research exists because of their ease of implementation and their substantially reduced computational requirements. 
The most well known and comprehensive survey of scheduling heuristics is by Panwalker and Iskander (1977) where more than 100 dispatching rules were presented, reviewed and classified based on their processing time, arrival time, queue status, etc. A survey of 34 dispatching rules could also be found in Blackstone et al, (1982). A common conclusion found in both surveys is that no single priority rule dominates and provides consistently best results for different job shop situations. There have also been many instances where combinations of rules have been successfully used in job shop scheduling (Blackstone et al, 1982). This approach has two or more dispatching rules dynamically selected for each of the machines based on the shop floor's prevailing conditions.

Review of literature related to dispatching rules in job shop schedules reveals a focus either to introduce new dispatching rules to optimize the shop floor performance or to review and test the existing ones, both for different shop configurations and performance objectives. Advanced simulation tools have been widely adopted for this purpose. For instance, in the simulation study done by Waikar et al, (1995), ten different dispatching rules (FIFO, SPT, DDT, LWKR, MWKR, MWKR-P, MWKR/P, MOPNR, SLK/RO and RAN) were tested for different shop loads ranging from 70 to $85 \%$, with job arrival and processing times following exponential and normal distributions respectively. Waikar et al, (1995) considered two different sets of performance criteria based on the flow-time and tardiness. The results of this study showed that both SPT and LWKR perform well under different shop loads not only for mean flowtime, which resulted in lowering in process inventories. but also for total queue time and time spent in the system. 
On the other hand, a comparative study done by Chang et al, (1996) evaluated the performance of 42 different dispatching rules using a linear programming model. These rules were broadly categorized in six different categories based on the smallest processing time, the largest processing time, due date, number of operations, random rule and lastly on the queue status. In order to evaluate all the dispatching rules, seven different performance objectives were considered, which were further divided into two sets, based on completion time and tardiness. Their analysis indicated that the shortest processing time (SPT) related rules consistently performed well, while the longest processing time based rules consistently performed badly.

Similarly, Rajender and Holthaus, (1999) carried out two different types of comparative studies of dispatching rules in both flow shops and job shops. In the first study, operations were performed on all the machines, but missing operations were allowed for both the shops in the second study. The job shop studied had ten machines, number of operations ranging from $(2 \ldots 10)$, processing times uniformly distributed between $(1,49)$, shop loads ranging from 80 to $95 \%$ and exponentially distributed interarrival time. In their study, thirteen different dispatching rules such as (FIFO, AT, EDD, SPT, PT+WINQ, etc) consisting of both existing and new rules proposed by Rajender and Holthaus, (1999) were evaluated for seven different performance objectives. The objectives were based either on flow time or on tardiness. The results showed that for the mean flowtime criterion SPT, PT+WINQ and RR (a rule by Raghu and Rajendran, 1993) performed consistently well under different shop loads. For the same criteria with missing operations. PT + WINQ rules emerged on average to be the best. The study also showed that for a higher shop load PT+WINQ performs significantly better than the SPT rule. 
Almost all the papers cited above either introduced new dispatching rules or tested existing rules based on a validated simulation model developed to test the rule itself. Thus, simulation is one of the most common tools widely used by many researchers for testing new or existing dispatching rules.

Although for many years the only practical approximation methods were priority/ dispatching rules, the introduction of more powerful computers, as well as an emphasis on carefully designed, analyzed and implemented algorithms has allowed more novel approaches to be developed for solving job shop problems. One example of such an approach is the use of artificial intelligence (AI). AI is a sub field of computer science that is concerned with integrating biological and computer intelligence. It has fundamental origins from biological understanding and uses principles in nature to find solutions for various complex problems. There are a number of classes of AI techniques, some of which are expert/knowledge-based system, neural networks (training and learning), fuzzy logic, genetic algorithm search, etc.

As the development in solving job shop scheduling problems increased, the degree of intelligence and the knowledge required for solving such problems also increased. Figure 2.1 presents the degree of artificial intelligence required by different scheduling approaches (Sim et al, 1994).

Expert / knowledge based systems mainly consist of knowledge and an inference engine to operate on that knowledge base. The application of such systems can be seen in 


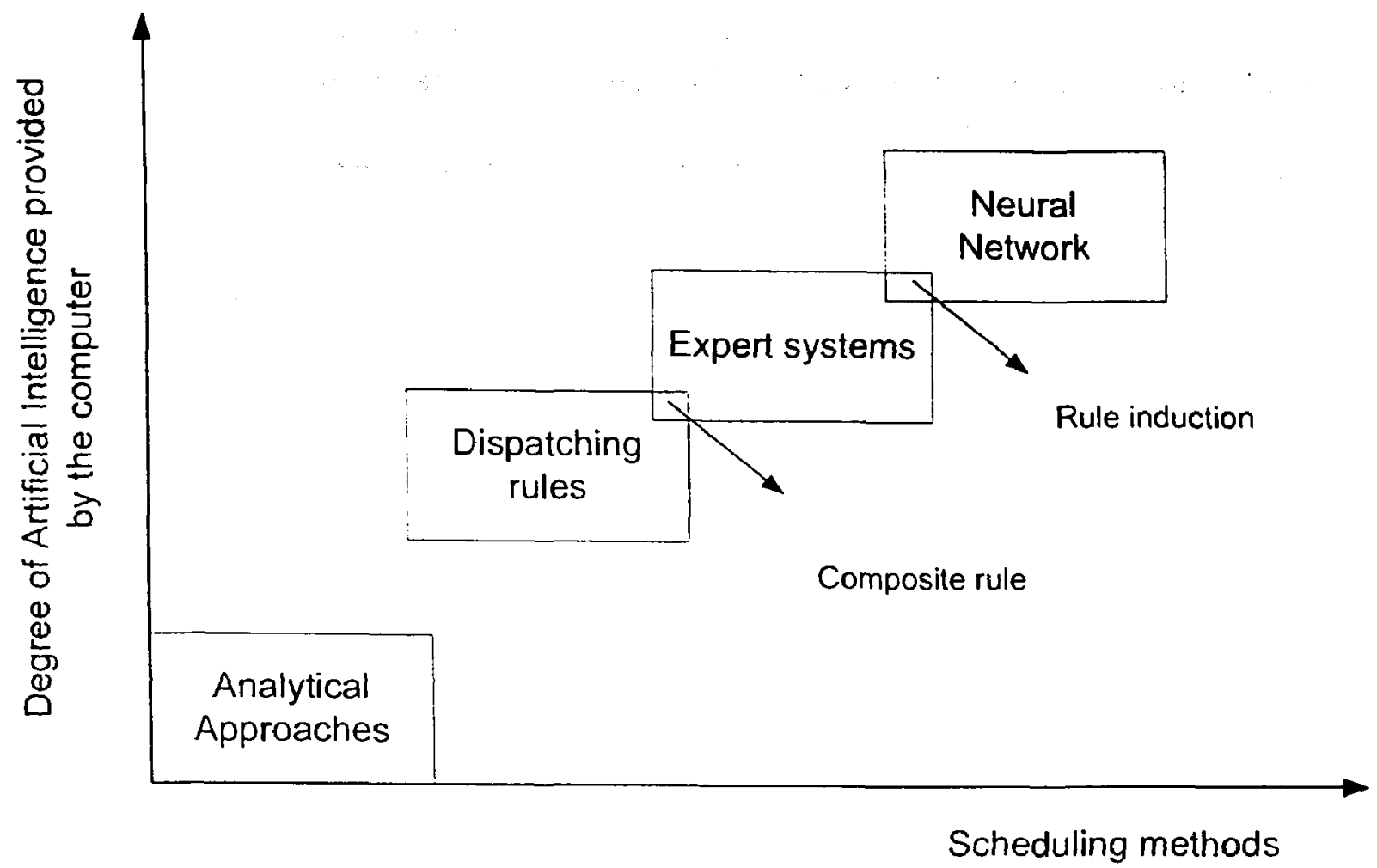

Fig. 2.1 Degree of artificial intelligence required by different scheduling approaches (Sim et al, 1994).

Pierreval, (1992), who demonstrated its use for selecting priority rules in a two machine flow shop model of a flexible manufacturing system, with the shop load of $83 \%$. Two different dispatching rules (SPT and EDD) for five different criteria (based on flow time and tardiness) were examined. The results showed that expert system (ES) provided on average good results for the performance criteria of mean flowtime and average waiting time as compared to SPT on both machines. Also, ES achieved the best performance among SPT-EDD and SPT-SPT combinations on the two machines for mean tardiness criteria.

Sim et al, (1994) developed an expert neural system in order to overcome some of the limitations with the expert system and for solving job shop problems for objectives related to tardiness. The artificial neural network was based on the back propagation feed forward neural network (BPNN) with the generalized delta rule as a learning algorithm and the sigmoid curve as the activation function. This model consisted of an input array 
of 14 neurons, sixteen sub-networks embedded in expert system and a single output node, which determined jobs to be processed first, based on the lowest output value. The job shop had nine resources, job arrivals following a Poisson distribution with an average rate of 0.6 to 0.775 . Each job had 3-6 operations and processing time of $1-4$ time units per operation and the performance objectives of minimizing lateness and tardiness. The first 10 input neurons represented different dispatching rules (such as SPT, LPT, EDD, etc) and the remaining neurons represented the arrival rate of jobs in the neural network. The results showed that the performance of this system was able to match the performance of the best dispatching rule used in training for both the performance objectives. Although the BPNN required a lengthy training process, once trained, the network only requires a single forward pass of computation (from the input nodes to the output node) to schedule.

Production demands are often cyclic in nature, and if the pattern of such demands can be recognized, then systems can respond to seasonal and sudden changes. Thus, a system that is able to recognize such patterns is in a position to update scheduling decisions effectively. Artificial neural networks, fuzzy logic and genetic algorithm are some of the methods that can be used in order to develop such systems.

Fuzzy logic application in dynamic selection of dispatching rules was examined by Subramaniam et al, (2000) for the performance objective of minimizing makespan in tenjob, ten-machine job shop problems. The proposed approach carefully selects three normalized input units, based on the conditions prevailing in the job shop, and a fuzzy scheduler selects an appropriate dispatching rule from the available candidates SPT, WINQ, MWKR to use for the individual machines. The results showed that the best makespan was obtained with the use of a combination of dispatching rules as compared to using a single dispatching rule such as SPT, LPT, MWKR, LWKR, FIFO, LIFO, 
MOPNR, LOPNR, NINQ, WINQ. The fuzzy scheduler is a one-pass approach and requires the same order of computation time as the simple dispatching rule.

On the other hand, both Kumar \& Srinivsan, (1996) and Chryssoluris \& Subramaniam, (2001) used genetic algorithms (GA) for dynamic selection of dispatching rules in job shop scheduling problems. In the former, a genetic search procedure for a real world combinatorial optimization problem was considered. The job shop had eighty jobs and fifty-nine machines, with the number of operations ranging from $(2,37)$. Some jobs could visit a machine more than once. Genetic algorithms use the idea of survival of the fittest by progressively accepting better solutions to the problems. In this case randomly generated strings of dispatching rules had a length of ren. The GA method generated a better makespan as compared to using any one of the seven different dispatching rules (SPT, LPT, TPT, RPT, DS, EDD, RS, FIFO) on all machines. The proposed algorithm yielded an improvement of about $3 \%$ in makespan over the best (SPT) among the seven rules tested, but the computation time required by the genetic algorithm (998 sec) was very large as compared to generating makespan by using a single dispatching rule (3.32 $\sec )$.

In the GA study by Chryssoluris \& Subramaniam, (2001), the dynamic job shop had six machines, fixed job arrival times, from three to six operations and processing time ranging uniformly from $U[1,100]$ and uniformly distributed due date of $\mathrm{U}[-100,1500]$ (loose) and $\mathrm{U}[-100,500]$ (tight). The study also considered machine breakdowns and repair, and jobs could visit the same resource more than once. The performance objectives were minimizing cost and tardiness. The proposed GA outperformed the other dispatching rules. Varying due dates did not seem to have any effect on the relative 
variation of the results. The computation time for this GA approach was about 2 orders of magnitude larger than for a simple dispatching rule.

An artificial neural network (ANN) has the capability to recognize and learn new pattems to generalize for any measurable function. ANN has been employed in a number of real world applications in manufacturing, finance, stock market, medical field, national security, etc. Zhang and Hung, (1995) provided a detailed survey on the neural networks in manufacturing with the applications in the areas of process planning, quality assurance, engineering design, scheduling, process control, etc.

There are various types of neural networks proposed and developed for solving scheduling problems. It has been observed in the literature that job shop scheduling problems were solved by neural networks either to obtain optimal sequence of jobs or to make a dynamic selection of dispatching rules based on the prevailing conditions of shop floor; so that the desired performance objective could be satisfied. For the purpose of research review, the various types of neural network that are of interest, viz:

1) Hopfield network and other optimizing networks

2) Multi-layer preceptrons (Back propagation networks)

Sabunguoglu, (1998) presented a detailed survey on using neural networks exclusively for scheduling applications. He proposed two different classifications, based on the types of neural networks used and the application area.

Hopfield networks were the first type of artificial neural network used for solving job shop scheduling problems. Foo and Takeji, (1988a, 1988b) proposed a Hopfield network with only input and output neurons. They mapped the problem on a two dimensional matrix of neurons with $(n m+1)$ rows and $(m n)$ columns, where $m$ is the number of machines and $\mathrm{n}$ is the number of jobs. A Simulated annealing process was then 
applied to the model in order to force the network out of local minima. The method was successful for a 4-job, 3-machine problem, but it had several limitations, such as the number of jobs must be greater than the number of machines. The proposed network in this method is not practical for large size problems and there is no guarantee of an optimal solution. Furthermore, computation time, even for a small problem is excessive.

Satake et al, (1994) used a Hopfield network for minimizing the makespan in a job shop by obtaining an optimal sequence of jobs. In this case, a Boltzmann mechanism was used in order to avoid local minima. Various problems were considered ranging from 4jobs/3-machines to 10-jobs/10-machines. The proposed network produced optimal or near optimal schedules within a reasonable amount of time. Further development in the application of Hopfield networks for solving a job shop problem can be obtained from (Sabunguoglu, 1998) and (Jain and Meeran, 1998).

The conclusions drawn from the review of Hopfield networks are that they require an excessive number of neurons and interconnections and can get easily trapped in local minima. For these reasons, they are suitable only for small size problems.

It has been observed in the literature that back propagation (BP) networks have drawn the attention of many researchers. One of the reasons for this interest is that BP network provides an increased speed for the selection process that may be needed in real world applications. Jones and Rableo, (1990) were the first to use the back propagated neural network in a proposed integrated (expert and ANN) scheduling system for ranking a set of dispatching rules based on the current shop status and job characteristics such as job types, arrival patterns, process plans. The output of the neural network was evaluated by an expert system, which then generated a schedule for the performance objective of 
minimizing tardiness. In their results, the BP network was able to predict correct results $90 \%$ of the time.

Pierreval et al, (1992) used both neural networks and simulation for selecting dispatching rules dynamically in a two-machine flow shop. In this study, five different dispatching rules and five different performance criteria were considered based on DR were considered. In this proposed back propagated neural network, there were 4 input units representing mean arrival rate of jobs, mean expected processing time on each machine and processing time variance, and a total of 19 output units representing a combination of five dispatching rules on both the machines. A trial and error approach determined 16 hidden neurons for a single hidden layer. A total of 500 training sets were used for training the network. The trained network was capable of selecting the dispatching rules based on the performance criteria. In a comparison of results between neural network (NN) and simulation, the authors highlighted that NN had the advantage of computational time over simulation for decision making in real time scheduling and, also, memory required by NN was less as compared to the simulations using SIMAN IV and GPSS packages. In a comparison between $\mathrm{NN}$ and expert system, the expert system required expertise to develop larger knowledge bases, and this was difficult to obtain in the case of selection of scheduling heuristics. The learning capabilities of NN avoid these problems.

During the literature review it has been ooserved that although neural networks were used in selection of dispatching rules in flow shops, there have been no investigations of their use in dynamic selection of combinations of dispatching rules in job shops. 
Thus, in the current research an artificial neural network to select combinations of dispatching rules is investigated. The task of the neural network is to pick an appropriate DR from a number of alternatives, given an instantaneous environment in the job shop. (Pierreval, 1992) Also, it is desired to test the suggestion of and Sabunguoglo, (1998) regarding the generalization propenty of back propagated networks for solving large size problems, having learned to solve small size problems. 


\section{CHAPTER 3}

\section{Artificial Neural Networks}

The objective of this chapter is to provide background information on artificial neural network, types of neusal networks and a detailed description of a back propagated neural network (BPNN).

\subsection{Introduction to Artificial Neural Networks (ANN)}

A job shop problem can be solved by several methodologies including mathematical programming, simulation, priority/dispatching rules, expert system, artificial intelligence (AI) etc. An Artificial Neural Network (ANN), which is one of the Al techniques, is an information-processing paradigm. The inspiration of using neural networks lies in its ability to extract information from complex data, similar to the biological nervous systems, and how the brain processes information. The key element of this paradigm is the novel structure of the information processing system. It is composed of a large number of highly interconnected processing elements (neurons) working in harmony to solve specific problems. ANN systems, like people, learn by examples and dynamically modify themselves to fit the data presented.

\subsubsection{Basic Concepts}

The basic building block of any ANN is the neuron, which is the fundamental cell of the brain or simply the processing unit of our brain. These neurons have three principal 
components: dendrites, the cell body and an axon, which are presented in Fig 3.1. The dendrites are tree like receptive networks of nerve fibers that carry electrical signals into the cell body. The cell body effectively sums and thresholds these incoming signals. The axon is a single long fiber that carries the signal from the cell body out of other neurons. The point of contact between axon of one cell and a dendrite of another cell is called a synapse. Learning process in the brain occurs due to the strengthening and weakening of synapses (Reinhardt, 1990).

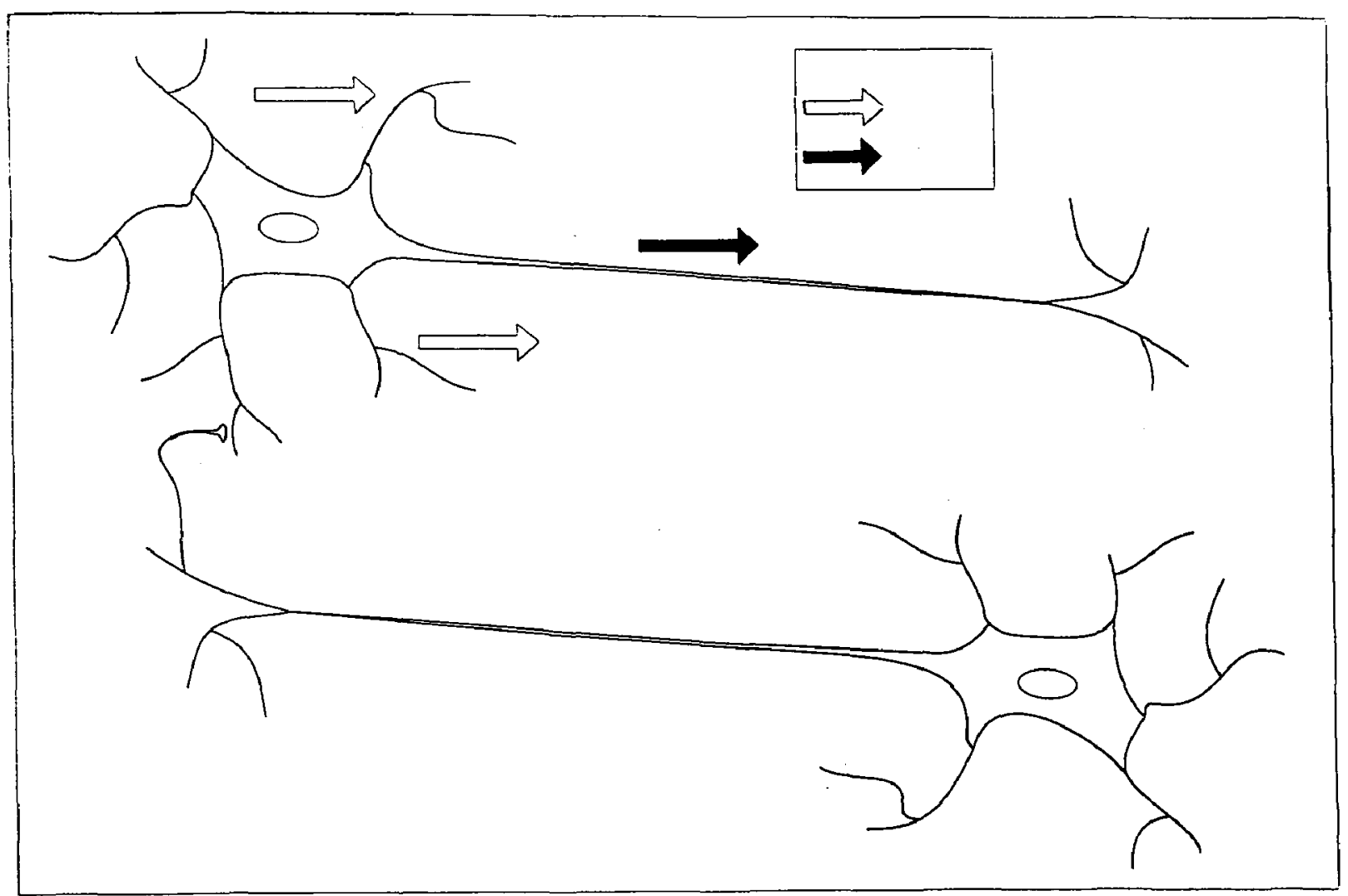

Fig 3.1 Basic features of a single biological neuron (based on Garson, 1998).

\subsubsection{Architecture of ANN}

ANNs are composed of basic units called processing elements (PE), which are also called as nodes or neurons. For example, in the back propagation (feed forward) network the PE are arranged in layers referred to as input, hidden and output layers. Each PE 
receives an input $X_{a}$ from every other neuron $a$, which is multiplied by corresponding weights. The aggregate input signal to the PE, Net, is calculated as the sum of these weighted inputs. (Smith, 1999).

The resulting signal is then passed through an activation function, which could be linear, logistic, step, ramp, sigmoidal or hyperbolic tangent depending on the problem to be solved. The output $O_{b}$ of the PE neuron is therefore $O_{b}=\mathrm{f}(\mathrm{Net})$.

In simple terms, neurons multiply an input by a set of weights, then combine these weighted inputs into an internal activity level by adding them together. The resulting signal is then modified by the transfer function of the PE to produce the output. Figure 3.2 shows the structure of a PE. The power of neural computation comes from the immense number of interconnections between the PEs, which share the load of the overall processing task, and also from the adaptive nature of the parameters (weights) that interconnect the PEs. (Garson, 1998).

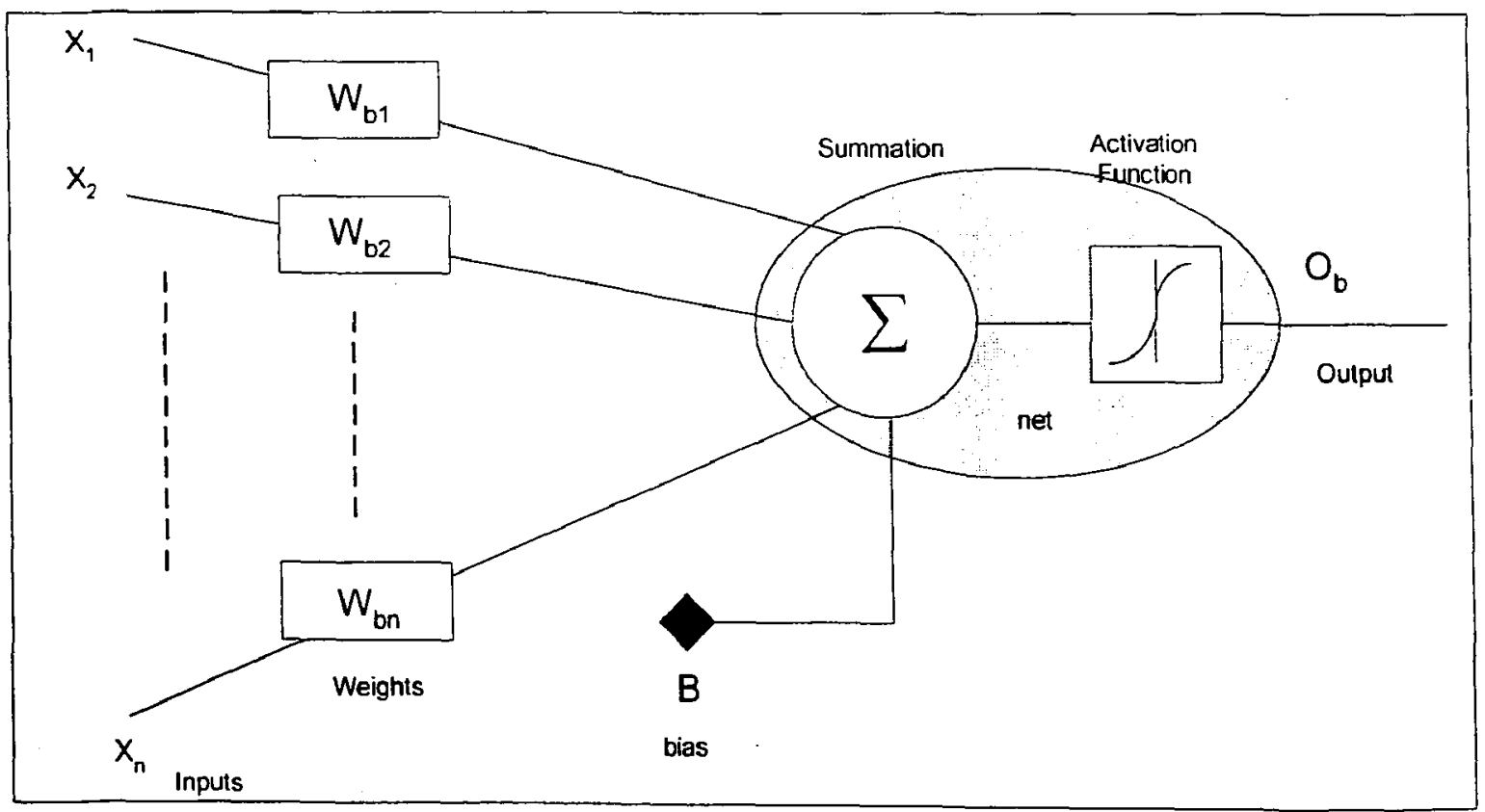

Fig 3.2 Processing elements (Garson, 1998). 


\subsubsection{Classification of Neural Network Architectures}

There are several neural network architectures, which are used not only for solving job shop scheduling, but also for several other applications. Figure.3.3 provides classifications within the field of neural computing. Jain and Meeran (1998) have presented details about each of the different types of neural networks. There are several different neural architectural models, but most of these can be divided into two main categories viz. feed forward and feedback.

Neural Network Architectures

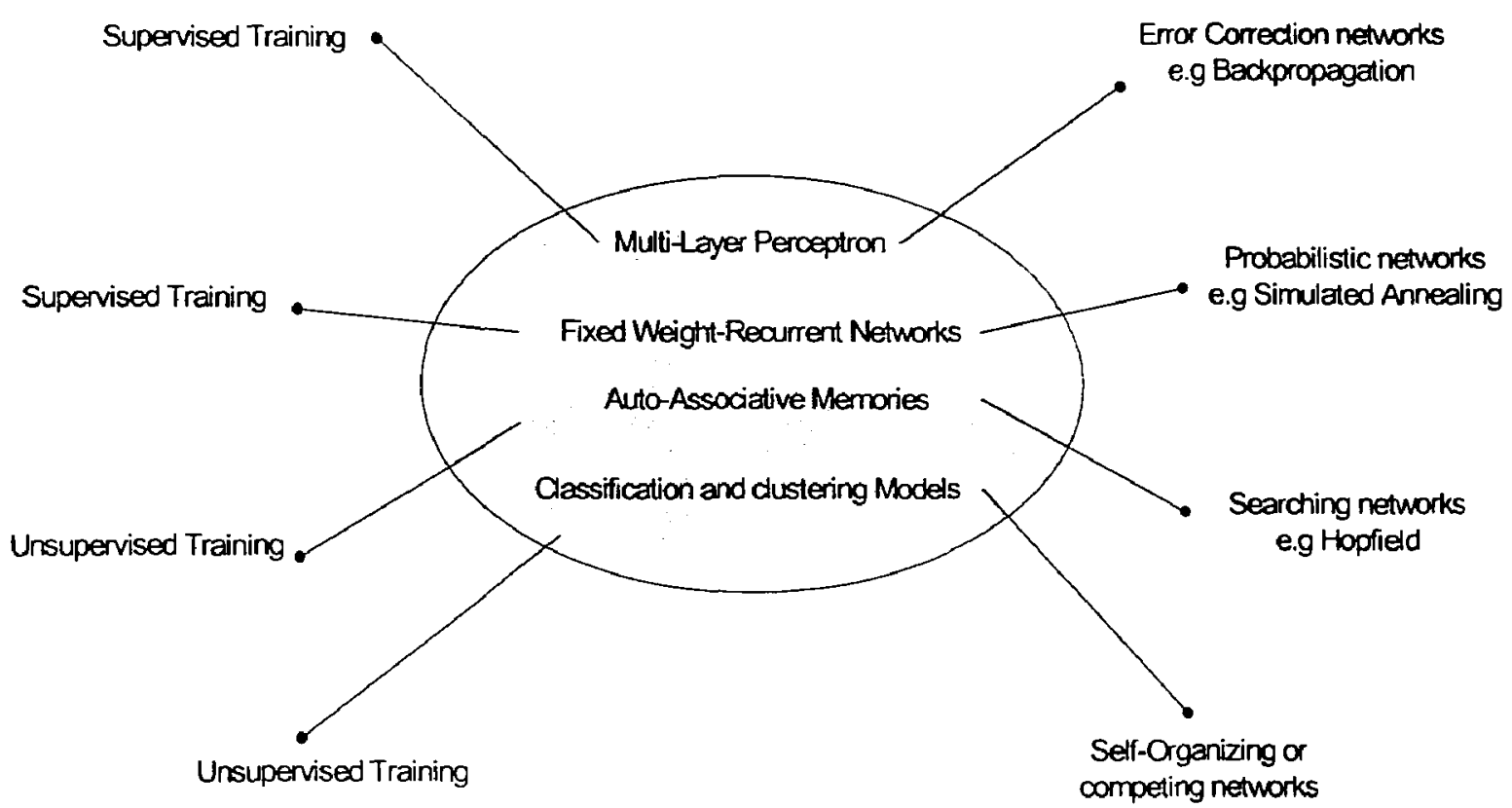

Fig.3.3 Classification of common neural network architectures (Jain and Meeran, 1998).

\subsubsection{Feed Forward Neural Network}

Processing elements are usually organized in layers. These layers can be connected in a number of different ways. A neural network with more than one layer is called a multi-layer neural network. Figure 3.4 shows the architecture of a multi-layer neural network. As can be seen in this network, the output from each layer feeds the next layer 
of units in a forward direction and there is no feedback connection between the layers, that is, the information is processed from left to right only. Garson (1998) describes this network as one that "has one or more inputs which are propagated through one or more number of hidden layers. Each layer contains a variable number of nodes, which finally reaches the output layer containing one or more output nodes".

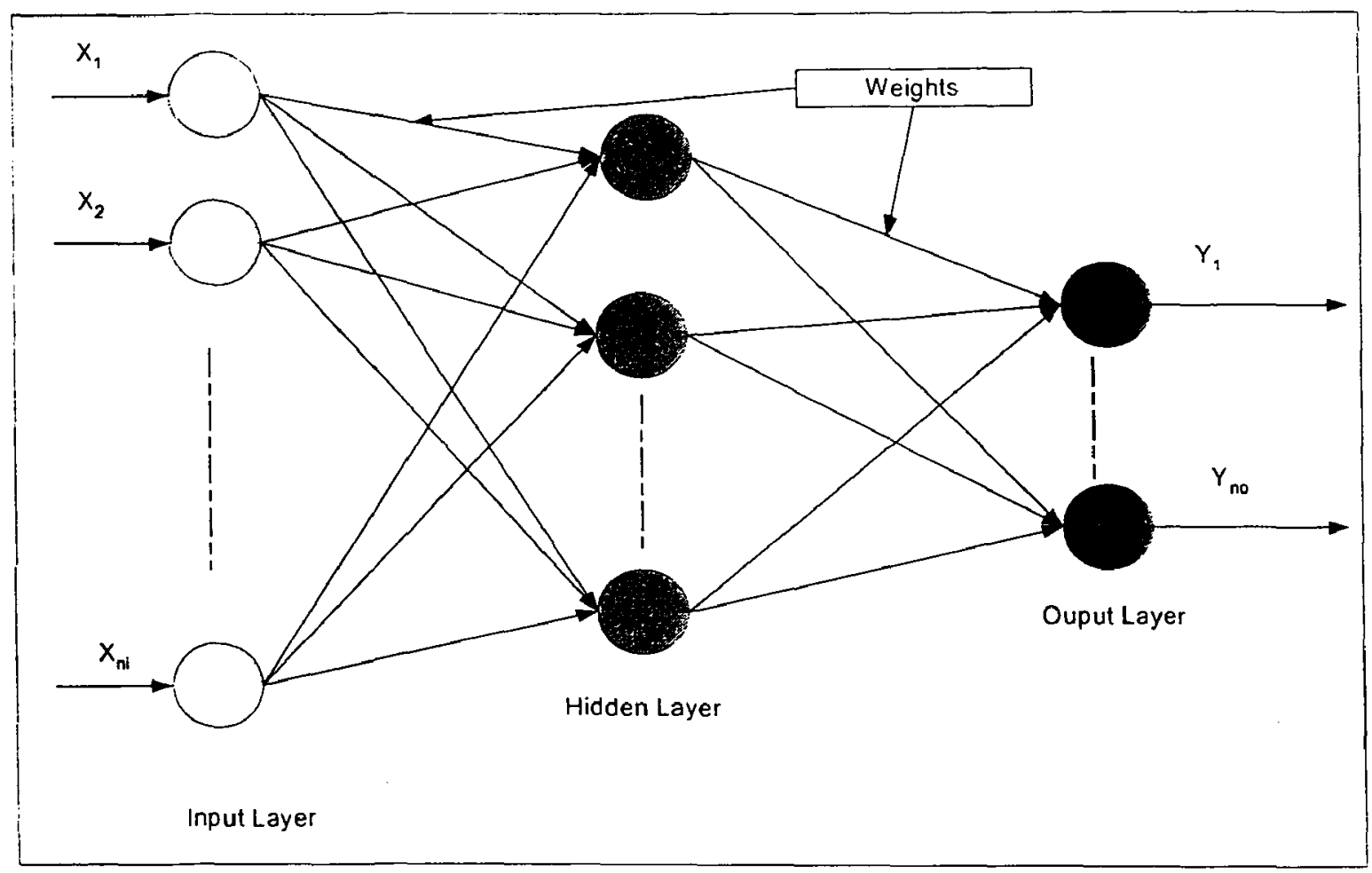

Fig 3.4 Multi-layer feed forward neural network (based on Garson, 1998).

\subsubsection{Learning Rule}

There are several learning rules, such as Hebb's rule, the Delta rule, Gradient Descent rule, etc. The choice of learning rule depends to some extent on the chosen architecture (Smith, 1999). For instance, in case of back propagation, the Delta rule is mostly used for generalization of errors. Learning rules can be divided into two main types: supervised and unsupervised learning (Smith, 1999). 
a) Supervised learning in simple terms is defined as 'learning with teacher' (Smith, 1999). In this case, the network is trained with the correct/desired responses for given inputs. During the learning process global information may be required. Usually, supervised learning is done off-line i.e. the network can be trained separately and later on the trained network is used for obtaining solutions to new problems (Garson, 1998).

b) Unsupervised learning. In this situation there is no external teacher used by the neural network for training. In other words, the desired output for the input is either unknown or does not exist, and learning is based on local information. Usually unsupervised learning is performed on-line (Smith, 1999).

\subsection{Neural Network training by Back Propagation (BPNN)}

Back propagation Neural Networks are a class of feed forward neural networks with supervised learning rules. In order to train a neural network to perform a certain task the weights of each unit must be adjusted in such a way that the error between the desired output and the actual output is reduced. The actual response of the network is subtracted from a target response to produce an error signal. The derivates of the output error are passed back to the hidden layer using the original weighted connections. Each hidden node then calculates the weighted sum of the back-propagated errors to find its indirect contribution to the known output errors. After each output and hidden node finds its error values, the weights are adjusted to reduce the errors. This process requires that the neural network compute the error derivate of the weights.

The back propagation algorithm is the most widely used method for determining error derivates of the weights. For the present research different BPNN networks are built for different performance objectives. There are a few important parameters and 
guidelines required while building the BPNN network, the details of which are provided below:

\subsubsection{Scaling Function}

Scaling function is used in the input layer to scale the data from their numeric range into a range that the network deals with efficiently. In case of supervised feed forward back propagation neural network the output patterns also require normalized or scaled value in the same range as of input. Common ranges for scaling are either 0 to 1 or -1 to 1 . In this study both the input and output patterns for neural network training are scaled between 0 and 1 (Neuroshell 2 manual; Swingler, 1999).

\subsubsection{Transfer Function}

In simple terms, the transfer function, which is also called as transformation, squashing, activation or threshold is a mathematical formula that determines the output of a processing neuron. In most areas of research the Sigmoid or S-shaped function is more popular as compared to other functions such as hyperbolic, tangent, step, ramping, arc tan or linear. For sigmoid function, the output varies continuously but not linearly as the input changes. According to Swingler (1999), the activation functions are "necessary to introduce non-linearity in the network. This non-linearity makes it possible to learn nonlinear functions". Sigmoid functions are more inclined to vary an output than threshold functions, because threshold functions usually are not sensitive to small changes in weights. For this reason, sigmoid functions are used in neuron activations for the proposed BPNN for job shop dispatching rules selection model (Garson, 1998). 


\subsubsection{Delta Rule}

"For a given input vector, the output vector is compared to the correct answer. If the difference is zero no learning takes place; otherwise, the weights are adjusted to reduce this difference" (Garson, 1998). The change in weight $w$ for output layer neuron $a$ with respect to input layer neuron $b$ is the learning rate $r$ times the activation function (f) for neuron $a$ times $c$, the correct desired output of neuron $a$ :

$$
\Delta w_{a b}=r f_{a b} c_{a}
$$

$C_{a}$ is the difference between the expected and actual output. During this learning the delta weight as shown in the equation is added to the existing weight $w_{a b}$. More details about the rule can be obtained from (Garson, 1998).

\subsubsection{Type of Datasets}

It is important to define and discuss the various types and sets of data. Data for neural networks are an input-output model. "Inputs are the presumed predictor variables, while outputs are the neural model's estimates of the dependent variable or variables" (Garson, 1998). In simple terms the data set is a set of examples for learning, which is nothing, but a "training set". The neural modeling software uses this set to compute model weights. Garson, (1998) defined the test data set as "the set of data to which the final neural model, and its associated weights, is applied for purposes of generalization."

There is a distinction in types of data sets between test sets and validation sets. According to Garson (1998), a test set is a collection of data that is used for testing the performance measure without changing any of the parameters, while a validation data set is used to tune the parameters. However, Garson, (1998) also mentioned that the test data 
sets are used for validation in the final stage of development of a neural model and thus, test data sets are sometime referred to as validation data sets. In this study only training and testing data sets are considered.

\subsubsection{Learning Rate and Momentum}

The Jearning rate $(\eta)$ controls the magnitude of the adjustments to the network's weights in response to the error. Each time a pattern is presented to the network, the weights leading to an output node are modified so as to produce a smaller error the next time the same pattern is presented. The magnitude of the weight adjustments is determined by the relationship: learning rate times the error. In most neural packages the learning rate is between 0.1 and 1 . In Neuroshell 2 the default value is 0.1 . A high learning rate might lead the local minimum to be overstepped constantly, causing oscillation from side to side, and never reaching convergence to the lower error state (Garson, 1998).

One way to allow faster learning without oscillation is to make the weight change a function of the previous weight change in order to provide a smoothing effect. The momentum $(\alpha)$ factor determines the proportion of the last weight change that is added into the new weight change (Garson, 1998). The Momentum, which causes the weight changes to be affected by the size of previous weight changes, is used to avoid local minima. Typically $\alpha$ is selected in the range of $0 \leq \alpha \leq 1$ " (Swingler, 1999). The default momentum value in Neuroshell 2 is 0.1 . 


\subsubsection{Initial Weights}

All network weights must be set to initial values before training starts. If weights are too large then the network might become unstable and the nodes saturated, and if weights are too small then weight changes will be slow. The choice of initial weights is dependent upon the problem and normalization of variables (Swingler, 1999). Generally, weights are chosen randomly. The default initial weight value in Neuroshell 2 is 0.3 .

\subsubsection{Neural Network Training}

Garson, (1998) defines training as, "the process of refining the weights in a neural model through a process in which training data set are fed into the model, analyzed and reprocessed through a number of iterations." The objective of training a neural network is to adjust the weights so that application of a set of inputs produces the desired set of outputs. Training assumes that each input vector is associated with an output vector.

In brief, training a feed-forward neural network with supervised learning consists of the following procedure, adapted from Garson (1998).

- Select a training pair from the training set and apply the input vector to the network input.

- Calculate network output using a forward pass. Calculation of network output is accomplished by using a feed-forward process and application of an activation

function for each layer in the network.

- Compute the difference between network output and the desired target value from the training pair output value.

- Change the network weights in a way that minimizes the error. 
This chapter has provided a detailed description of BPNN along with different parameters to be considered while designing BPNN for solving the job shop problem for two different performance objectives. The next chapter describes building a BPNN for selecting appropriate dispatching rules in a 5-machine job shop. 


\section{CHAPTER 4}

\section{Methodology and Design of BPNN}

This chapter introduces the methodology for constructing a back propagation neural network (BPNN) that selects a combination of dispatching rules to use in a job shop problem.

\subsection{Designing a BPNN for job shop scheduling}

In order to build a BPNN, there are some common steps that should be followed for achieving desired objectives. Baily and Thompson, (1993) provided detailed guidance on how to build neural networks along with the design decisions to be considered for commonly used neural paradigms like BPNN, Boltzman machines, Hopfield network, etc. Likewise, Kaastra and Boyd, (1996) provided a step-by-step guide for building a BPNN forecasting model. Based upon both these references, Figure. 4.1 is constructed to highlight the main steps required in designing a BPNN. The procedure is not usually a single pass process and some of the steps like training and testing may need to be carried out several times.

The material in this section is organized based on the design steps of Figure. 4.1. The detailed explanation is presented for a BPNN with regards to the performance criterion of minimizing makespan. This same procedure can be used for other performance criteria, as will be illustrated for minimizing the mean flowtime in section 4.7 of this chapter. 


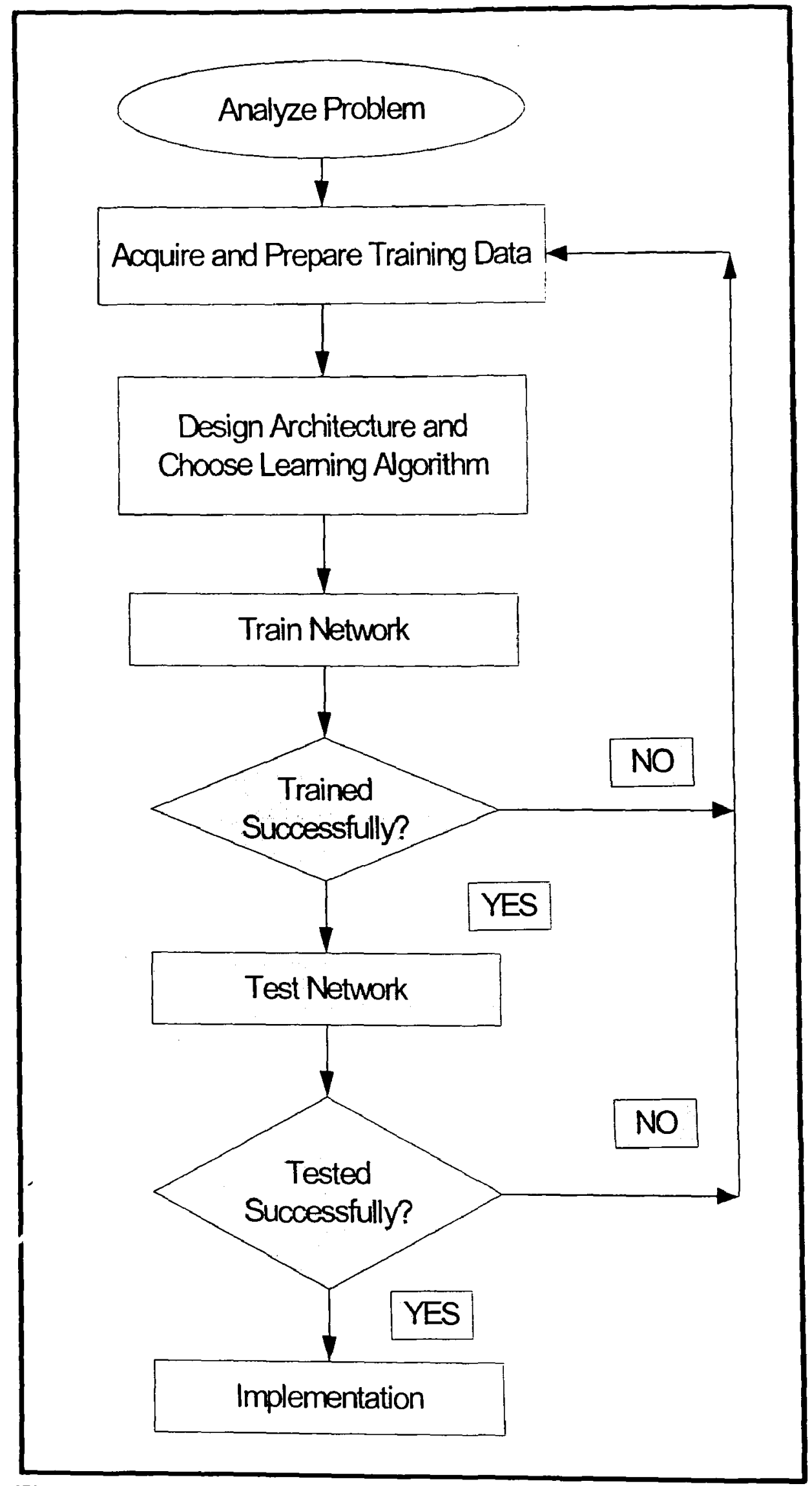

Fig 4.1 Design methodology for building ANN. 


\subsection{Problem Analysis}

The definition, nature and scope of the job shop problem along with the type of neural network recommended for solving it have been discussed in chapter one. The case of a 5-machine job shop is considered, although a similar approach can be used for job shops having different numbers of machines. The objective is to select for each machine, one of three alternative dispatching rules so that a given performance criterion is optimized for the problem on hand.

\subsection{Data Acquisition and Preparation}

In building an effective ANN, the researcher has to decide what kind of data are to be used, namely either historical or constructed data. If historical data are being used, then they must be sorted or filtered from noisy data, if any. A format and range for each input and output are then selected and the data are expressed in a manner that can be presented to the BPNN. This usually takes form of a data vector as follows:

$<$ Input 1 , input 2 , input $3, \ldots \ldots$..jnput $\mathrm{N}_{\mathrm{j}}$, output 1 , output 2 , output $3 \ldots \ldots$ output $\mathrm{N}_{\mathbf{0}}>$

Where $\mathrm{N}_{j}$ and $\mathrm{N}_{\mathrm{O}}$ represents number of input and output nodes respectively.

Before the data are collected, it is necessary to decide which of the job shop attributes will be used in the input layer and what output information is desired of the network.

\subsubsection{Input Variable Selection}

Input variables must be selected very carefully, taking into account the given performance objective to be achieved. Here, the objective considered is to minimize 
makespan, which mainly depends on the job's processing times $\left(P_{i j k}\right)$ and the routes $\left(Q_{i k}\right)$. However, in a case where every job visits each machine once, the subscript $j$ can be dropped and the processing time represented as $\left(P_{i k}\right)$. The number of units in the input vector is dictated by the specific representation adopted for the schedule of the job shop problem. In the proposed representation, it is desired to characterize the job shop in terms of machine loads, dispersion of machine processing times and mean routing order on each machine. This is achieved by a total of 15 input units for a 5 -machine job shop. The information carried in each of these units is organized as follows:

lnput 1: - Total processing time on machine $1 \quad=\sum_{i=1}^{n} P_{i 1}$

Input 2: - Total processing time on machine $2=\sum_{i=1}^{n} P_{i 2}$

Input 3: - Total processing time on machine $3=\sum_{i=1}^{n} P_{i 3}$

Input 4: - Total processing time on machine $4 \quad=\sum_{i=1}^{n} P_{i 4}$

Input 5: - Total processing time on machine $5=\quad=\sum_{i=1}^{n} P_{i 5}$

Input 6: - Variance of processing time for machine $1=\frac{n \sum_{i=1}^{n} \mathrm{P}_{i 1}-\left(\sum_{i=1}^{n} \mathrm{P}_{\mathrm{i} 1}\right)^{2}}{n(n-1)} \ldots$

Input 7: - Variance of processing time for machine $2=\frac{n \sum_{i=1}^{n} \mathrm{P}_{\mathrm{i} 2}-\left(\sum_{i=1}^{n} \mathrm{P}_{\mathrm{i} 2}\right)^{2}}{n(n-1)} \ldots$

Input 8: - Variance of processing time for machine $3=\frac{n \sum_{i=1}^{n} \mathrm{P}_{\mathrm{i} 3}-\left(\sum_{i=1}^{n} \mathrm{P}_{\mathrm{i} 3}\right)^{2}}{n(n-1)} \ldots$ 
Input 9: - Variance of processing time for machine $4=\frac{n \sum_{i=1}^{n} \mathrm{P}_{\mathrm{i} 4}-\left(\sum_{i=1}^{n} \mathrm{P}_{\mathrm{i} 4}\right)^{2}}{n(n-1)} \ldots$

Input 10: - Variance of processing time for machine $5=\frac{n \sum_{i=1}^{n} \mathrm{P}_{\text {is }}-\left(\sum_{i=1}^{n} \mathrm{P}_{\text {is }}\right)^{2}}{n(n-1)} \ldots$

Input 11: - Mean routing order of machine $1=\mathrm{R}_{\mathrm{k}=1}=\frac{1}{n} \sum_{i=1}^{n} \mathrm{Q}_{\mathrm{i1}}$

lnput 12: - Mean routing order of machine $2=\mathrm{R}_{\mathrm{k}=2}=\frac{1}{n} \sum_{i=1}^{n} \mathrm{Q}_{\mathrm{i} 2}$

Input 13: - Mean routing order of machine $3=\mathrm{R}_{\mathrm{k}=3}=\frac{1}{n} \sum_{i=1}^{n} \mathrm{Q}_{i 3}$

lnput 14: - Mean routing order of machine $4=\mathrm{R}_{\mathrm{k}=4}=\frac{1}{n} \sum_{i=1}^{n} \mathrm{Q}_{\mathrm{i4}}$

$\ldots$

Input 15: - Mean routing order of machine $5=\mathrm{R}_{\mathrm{k}=5}=\frac{1}{n} \sum_{i=1}^{n} \mathrm{Q}_{\mathrm{i}}$

\subsubsection{Construction of an Input Vector}

A single input vector represents a job shop problem. This section gives detailed illustration of how an input vector is computed for a randomly selected 10-job, 5-machine example. Table 4.1 depicts the raw data for this problem, identified as N10M265 - 10 denotes a 10-job problem; $M$ represents the makespan criterion and 265 the problem number. Note this example problem is taken from a set of 2494 random problems generated for training the network). The details of how each of the input units is constructed is explained hereafter. 


\begin{tabular}{|c|ccccc|ccccc|}
\hline $\begin{array}{c}\text { Job } \\
\text { No }\end{array}$ & \multicolumn{5}{|c|}{ Routes } & \multicolumn{4}{|c|}{ Processing time $\left(P_{i j k}\right)$ time units } \\
\hline 1 & 3 & 1 & 4 & 5 & 2 & 47 & 67 & 43 & 74 & 57 \\
2 & 3 & 4 & 5 & 2 & 1 & 47 & 44 & 72 & 74 & 60 \\
3 & 4 & 1 & 2 & 5 & 3 & 46 & 85 & 84 & 69 & 30 \\
4 & 3 & 4 & 5 & 2 & 1 & 43 & 31 & 55 & 51 & 85 \\
5 & 1 & 4 & 5 & 3 & 2 & 77 & 24 & 55 & 40 & 88 \\
6 & 1 & 4 & 5 & 3 & 2 & 95 & 17 & 78 & 52 & 63 \\
7 & 1 & 4 & 5 & 3 & 2 & 64 & 48 & 53 & 33 & 49 \\
8 & 4 & 1 & 2 & 5 & 3 & 41 & 66 & 66 & 53 & 37 \\
9 & 3 & 5 & 1 & 2 & 4 & 52 & 64 & 95 & 60 & 21 \\
10 & 3 & 4 & 5 & 2 & 1 & 46 & 52 & 64 & 53 & 85 \\
\hline
\end{tabular}

Table 4.1 Raw data of sample problem N10M265.

Input units $]$ through 5 (Total processing times):

The total processing time on a given machine is the summation of processing time requirements on that machine. This measure helps to identify which machine is a bottleneck and to what degree. Total load on each of the machines is obtained by adding the processing time of all the jobs visiting that machine. The bottleneck machine is that which has the highest load. Applying equations 4.1 through 4.5 for the data of Table 4.1 results in total machine loadings given in the third column in Table 4.2. Here, machine 1 with a total load of 780 (time) units, is the bottleneck machine.

\begin{tabular}{|c|c|c|c|}
\hline Input unit & Machine & Total load & Normalized value \\
\hline 1 & 1 & 780 & 1 \\
2 & 2 & 645 & 0.827 \\
3 & 3 & 427 & 0.547 \\
4 & 4 & 367 & 0.471 \\
5 & 5 & 637 & 0.817 \\
\hline \multicolumn{2}{|c|}{ Max Value (H) $=$} & 780 & \\
\hline
\end{tabular}

Table 4.2 Determining inputs 1 to 5 for example no. NM10265.

The next step is to convert the machine loads in the range $(0,1)$. This is necessary because the input layer must have continuous values between 0 and 1 whenever the sigmoid activation function is used. This can be achieved by calculating a relative percentage for each load with respect to the highest load. Thus, for a given range of data with maximum values $=\mathrm{H}$, a specific normalized value $\mathrm{V}$ is calculated by $\mathrm{V} / \mathrm{H}$. The 
normalized machine loads for the example job shop of Table 4.1 are given in the last column of Table 4.2

Input units 6 through 10 (Variance of processing time):

As different jobs have different processing times on a given machine, it is useful to have an indication of the spread (dispersion) of these times about the mean. This is achieved by calculating variance, which is a measure of this spread for each machine. The variances for the sample problem in Table 4.1 are computed by using equations 4.6 to 4.10 . The data are formatted in the range 0 to 1 by dividing them by the maximum variance among the five machines. Table 4.3 shows the variance for the five machines of the problem given in Table 4.1 and the normalized values used for input units 6 through 10.

\begin{tabular}{|c|c|c|c|}
\hline Input unit & Machine & Variance & Normalized value \\
\hline 6 & 1 & 169.56 & 0.92 \\
7 & 2 & 184.28 & 1.00 \\
8 & 3 & 57.34 & $0.31]$ \\
9 & 4 & 154.23 & 0.837 \\
10 & 5 & 87.56 & 0.475 \\
\hline \multicolumn{2}{|c|}{ Max Value $=$} & 184.28 & \\
\hline
\end{tabular}

Table 4.3 Inputs 6 to 10 for example problem N10M265.

Input Units 11 through 15 (Mean order of routing $\left(\mathrm{R}_{\mathrm{k}}\right)$ :

The purpose of this measure is to characterize the prevailing flow pattern, if any, due to the combination of job routes for the problem to be scheduled. The mean routing order $\left(R_{k}\right)$ for each machine $k$ is calculated as follows: -

$\mathrm{R}_{\mathrm{k}}=\frac{1}{n} \sum_{\mathrm{i}=1}^{n} \mathrm{Q}_{\mathrm{ik}}$

Where, $Q_{i k}=$ machine $k$ 's order in job $i$ 's route.

$$
k=1,2, . ., 5
$$


The minimum value for $Q_{i k}$ is 1 and the maximum is 5 (because 5 machines are visited by each job). A lower value for $Q_{i k}$ indicates that machine $k$ is visited predominantly by jobs during the earlier stages in their routes, while higher values suggest that the machine is visited more towards the later operations of the job's processing orders. The mean routing order for five machines based on the problem of Table 4.1 is given in column 3 of Table 4.4

\begin{tabular}{|c|c|c|c|}
\hline Input unit & Machine & Mean routing order & Normalized Value \\
\hline 11 & 1 & 2.7 & 0.54 \\
12 & 2 & 4.2 & 0.84 \\
13 & 3 & 2.7 & 0.54 \\
14 & 4 & 2.2 & 0.44 \\
15 & 5 & 3.2 & 0.64 \\
\hline \multicolumn{2}{|c|}{ Max Value $=$} & 4.2 & \\
\hline
\end{tabular}

Table 4.4 Example of normalized value for inputs 11 through 15 for example problem N10M265.

From Table 4.4 it is seen, for example, that the mean routing order for machine 2 is 4.2. This indicates that machine 2 is predominately visited towards the end of the routes in most of the jobs. The mean routing orders are normalized between 0 and 1 by dividing $Q_{i k}$ for each machine by the number of machines ( 5 in this case).

Once all the input data computations are done, the input vector can be prepared. The 15 input units representing the 10 -job problem in Table 4.1 is constructed by using the normalized values from the last columns of Table 4.2, 4.3 and 4.4 . This result is shown in Table 4.5 .

\begin{tabular}{|c|c|c|c|c|c|c|c|c|c|c|c|c|c|c|c|}
\hline $\begin{array}{l}\text { Data } \\
\text { Set No. }\end{array}$ & \multicolumn{5}{|c|}{ Total Processing time } & \multicolumn{5}{|c|}{ Variance of Processing time } & \multicolumn{5}{|c|}{ Routing Complexity, } \\
\hline $\begin{array}{l}\text { Input } \\
\text { units }\end{array}$ & 1 & 2 & 3 & 4 & 5 & 6 & 7 & 8 & 9 & 10 & 11 & 12 & 13 & 14 & 15 \\
\hline value & 1.0 & 0.827 & 0.547 & 0.471 & 0.817 & 0.92 & 1.0 & $0.31]$ & 0.837 & 0.475 & 0.54 & 0.84 & 0.54 & 0.44 & 0.64 \\
\hline
\end{tabular}

Table 4.5 15 Unit input vector representing job shop problem of Table 4.1.

The input vector in Table 4.5 can be interpreted to describe the job shop problem, for instance, as one where the machine 1 is a strong bottleneck. Machines 1,2 and 4 have 
a wider range of processing times as compared to the other two machines; and finally machine 2 is visited last or near last by most of the jobs.

\subsubsection{Output Layer}

For solving the makespan minimization problem the three dispatching rules SPT, LPT and MWKR are considered. Past research by Kumar \& Srinivsan, (1996) and by Subramaniam et al, (2000) has showed rules SPT, LPT and MWKR are among a few rules effective in minimizing makespan. The neural network's task is to select one of these dispatching rules for each of the five machines. Therefore, the output layer has been designed with 15 units ( 3 rules $\times 5$ machines). Each unit represents a dispatching rule for a machine. The higher the value of an output unit, the higher is the desirability of using the dispatching rule associated with that unit on the corresponding machine.

\subsubsection{Data Collection}

Now that the input units and the desired output have been defined, the next step is to collect data for training and testing the network. A total of 7500 job shop problems were generated randomly using $n=10,15$ and 20 jobs in equal quantities. $\mathrm{A} C++$ program (see Appendix Al) is used for this purpose. The processing times used for the problems are uniformly distributed between $U(10,99)$ integer time units. The number of different job routes in each problem is between 5 and 11, selected randomly from the 120 (or 5!) possible routes in a 5-machine job shop. This range for the routes is used to ensure that several jobs have identical routes in order to simulate more realistic situations. It is 
unlikely in a real world application, particularly within group technology settings, for most if not all jobs to have different routes from one another.

The optimal combination of dispatching rules that minimizes makespan for a given 5-machine job shop problem is found by enumerating all possible combinations, a total of 243 possibilities of dispatching rules $\left(3^{5}\right.$ combinations) for the three dispatching rules SPT, LPT and MWKR. The neural output assumes values between 0 and 1.0; the maximum value " 1.0 " suggests undisputed preference for the specific dispatching rule represented by the unit. As the output value reduces, the preference for the corresponding dispatching rule diminishes proportionately. In supervised learning, desired (or target) outputs are needed in training the network. The target outputs for the proposed network are extracted from the optimal rule combinations in the following fashion, using data from Table 4.1 to illustrate the procedure.

The optimal selection of dispatching rules for the problem N10M265 gives a makespan of 826 . Two different optimal combinations of the three rules exist for this problem as shown in Table 4.6

\begin{tabular}{|c|c|c|c|c|}
\hline $\mathrm{M} / \mathrm{C} 1$ & $\mathrm{M} / \mathrm{C} 2$ & $\mathrm{M} / \mathrm{C} 3$ & $\mathrm{M} / \mathrm{C} 4$ & $\mathrm{M} / \mathrm{C} 5$ \\
\hline $\mathrm{MWKR}$ & $\mathrm{SPT}$ & $\mathrm{MWKR}$ & $\mathrm{LPT}$ & $\mathrm{LPT}$ \\
$\mathrm{MWKR}$ & $\mathrm{MWKR}$ & $\mathrm{MWKR}$ & $\mathrm{LPT}$ & $\mathrm{LPT}$ \\
\hline
\end{tabular}

Table 4.6 Optimal rule combinations for example problem N10M265.

In order to represent DR combination, the data of Table 4.6 are presented in a numerical form suitable for the output vector. The number of times a rule appears in an optimal combination is computed in each machine. For example, in machine 2 (Table 4.6) both SPT and MWKR (but not LPT) appear once in the optimal result. A similar calculation for the other machines is done and the final counts are given in Table 4.7. 


\begin{tabular}{|c|c|c|c|c|c|}
\hline DR & M/C 1 & M/C 2 & M/C 3 & M/C 4 & M/C 5 \\
\hline SPT & 0 & 1 & 0 & 0 & 0 \\
LPT & 0 & 0 & 0 & 2 & 2 \\
MWKR & 2 & 1 & 2 & 0 & 0 \\
\hline
\end{tabular}

Table 4.7 Numerical representation for optimal rule combination of Table 4.6.

In order to normalize the output results of Table 4.7 between 0 and 1 , the entries in Table 4.7 are divided by the total number of optimal results (2) for the problem. The resulting output $(\mathrm{o} / \mathrm{p})$ vector is shown in Table 4.8, which represents the target output pattern that would be associated for training purposes with the input vector of Table 4.5.

\begin{tabular}{|c|c|c|c|c|c|c|c|c|c|c|c|c|c|c|}
\hline & \multicolumn{2}{|c|}{ Machinel } & \multicolumn{3}{|c|}{ Machine2 } & \multicolumn{3}{|c|}{ Machine 3} & \multicolumn{3}{|c|}{ Machine4 } & \multicolumn{3}{|c|}{ Machines } \\
\hline SPT & LPT & MWKR & SPT & LPT & MWKR & SPT & LPT & MWKR & SPT & LPT & MWKR & SPT & LPT & MWKR \\
\hline 0.000 & 0.000 & 1.000 & 0.50 & 0.00 & 0.50 & 0.00 & 0.00 & 1.00 & 0.000 & 1.000 & 0.000 & 0.00 & 1.000 & 0.00 \\
\hline
\end{tabular}

Table 4.8. 15-unit vector representing desired output for example problem N10M265.

As can be seen from the output vector above, a minimum makespan can be obtained by using MWKR on machines 1 and 3; LPT on machine 4 and 5; and either SPT or MWKR with equal favor on machine 2. Table 4.9 shows a sample of several pairs of training patterns generated from the training problems where number of jobs $(n)$ is 10 . The optimal combinations of dispatching rule in each problem are used for training. In order to compute the neural input and output (optimal combinations of dispatching rule for each problem) vectors are computed by using a program in $\mathrm{C}++$ (see Appendix A2). The back propagation neural network model proposed for selecting one of the three DRs for each of the machines in a 5-machine job shop appears as shown in Figure 4.2.

\subsubsection{Validation of Training data set output}

In order to validate the makespan results obtained from the $\mathrm{C}++$ program of Appendix A2, a simulation model of a 5-machine job shop was developed with a student 


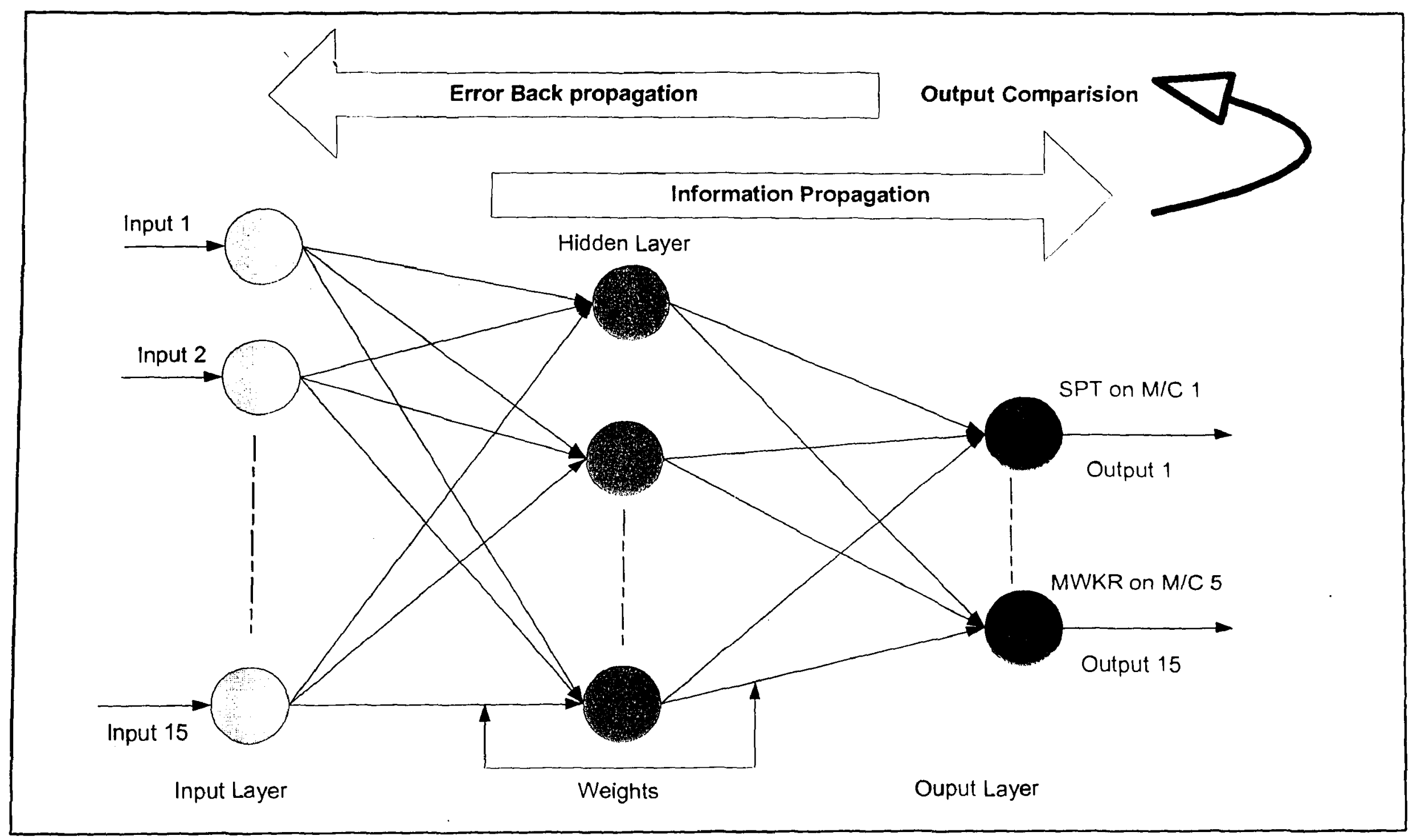

Fig. 4.2 Structure of ANN proposed for the 5-machine job shop. 


\begin{tabular}{|c|c|c|c|c|c|c|c|c|c|c|c|c|c|c|c|}
\hline Data Set & \multicolumn{5}{|c|}{ Total Processing time } & \multicolumn{5}{|c|}{ Variance of Processing time } & \multicolumn{5}{|c|}{ Mean Routing order } \\
\hline Numbers & Input 1 & Input 2 & Input 3 & Input 4 & Input 5 & Input 6 & Input 7 & Input 8 & Input 9 & Input 10 & Input II & Input 12 & Input 13 & Input 14 & Input 15 \\
\hline N10M301 & 0.50 & 0.71 & 0.80 & 0.93 & 1.00 & 0.75 & 0.98 & 0.51 & 1.00 & 0.46 & 0.46 & 0.60 & 0.76 & 0.60 & 0.58 \\
\hline N10M302 & 0.92 & 1.00 & 0.87 & 0.90 & 0.88 & 0.32 & 1.00 & 0.67 & 0.55 & 0.59 & 0.48 & 0.72 & 0.46 & 0.80 & 0.54 \\
\hline N10M303 & 0.75 & 0.35 & 1.00 & 0.67 & 0.72 & 0.64 & 1.00 & 0.58 & 0.54 & 0.42 & 0.68 & 0.36 & 0.50 & 0.58 & 0.88 \\
\hline N10M304 & 0.71 & 0.54 & 0.57 & 0.86 & 1.00 & 0.70 & 0.79 & 1.00 & 0.89 & 0.88 & 0.36 & 0.66 & 0.50 & 0.76 & 0.72 \\
\hline N10M305 & 1.00 & 0.88 & 0.63 & 0.94 & 0.82 & 0.74 & 0.97 & 1.00 & 0.60 & 0.77 & 0.32 & 0.60 & 0.60 & 0.76 & 0.72 \\
\hline $\mathrm{N} 10 \mathrm{M} 306$ & 0.53 & 0.38 & 0.97 & 1.00 & 0.48 & 0.43 & 0.47 & 0.22 & 1.00 & 0.37 & 0.52 & 0.64 & 0.62 & 0.66 & 0.56 \\
\hline N10M307 & 0.48 & 1.00 & 0.84 & 0.84 & 0.53 & 0.76 & 0.77 & 0.39 & 0.44 & 1.00 & 0.48 & 0.88 & 0.52 & 0.32 & 0.80 \\
\hline N10M308 & 0.87 & 0.87 & 1.00 & 0.98 & 0.40 & 0.48 & 0.69 & 0.63 & 0.58 & 1.00 & 0.46 & 0.60 & 0.78 & 0.44 & 0.72 \\
\hline N10M309 & 0.84 & 0.74 & 1.00 & 0.55 & 0.63 & 0.88 & 0.77 & 0.84 & 0.84 & 1.00 & 0.72 & 0.68 & 0.50 & 0.54 & 0.56 \\
\hline N10M310 & 1.00 & 0.66 & 0.81 & 0.63 & 0.91 & 0.76 & 0.61 & 1.00 & 0.47 & 0.52 & 0.62 & 0.72 & 0.38 & 0.74 & 0.54 \\
\hline N10M311 & 0.52 & 0.78 & 0.95 & 1.00 & 0.63 & 0.20 & 0.68 & 0.70 & 1.00 & 0.71 & 0.68 & 0.60 & 0.64 & 0.44 & 0.64 \\
\hline $\mathrm{N} 10 \mathrm{M} 312$ & 0.66 & 0.75 & 1.00 & 0.94 & 0.93 & 1.00 & 0.48 & 0.69 & 0.55 & 0.96 & 0.52 & 0.56 & 0.76 & 0.60 & 0.56 \\
\hline $\mathrm{N} 10 \mathrm{M} 313$ & 0.80 & 0.95 & 0.79 & 0.57 & 1.00 & 0.88 & 0.15 & 0.51 & 1.00 & 0.64 & 0.66 & 0.54 & 0.60 & 0.54 & 0.66 \\
\hline N10M314 & 0.75 & 0.66 & 0.61 & 0.96 & 1.00 & 1.00 & 0.85 & 0.72 & 0.84 & 0.77 & 0.30 & 0.76 & 0.6 .8 & 0.68 & 0.58 \\
\hline \multicolumn{16}{|c|}{ Target Output Patterns } \\
\hline Data Set & \multicolumn{3}{|c|}{ Machinel } & \multicolumn{3}{|c|}{ Machine2 } & \multicolumn{3}{|c|}{ Machine3 } & \multicolumn{3}{|c|}{ Machinc4 } & \multicolumn{3}{|c|}{ Machines } \\
\hline Number & $o / p 1$ & $o / p 2$ & $\alpha / p 3$ & $\mathrm{~d} / \mathrm{p} 4$ & $\mathrm{~d} / \mathrm{p} 5$ & $\mathrm{o} / \mathrm{p} 6$ & $o / p 7$ & $\mathrm{o} / \mathrm{p} 8$ & $o / p 9$ & $\alpha / p 10$ & o/p 11 & $\mathrm{~d} / \mathrm{p} 12$ & $\alpha / p 13$ & $\mathrm{~d} / \mathrm{p} 14$ & $\alpha / p 15$ \\
\hline N10M301 & 0.000 & 0.000 & 1.000 & 0.000 & 1.000 & 0.000 & 0.000 & 0.000 & 1.000 & 1.000 & 0.000 & 0.000 & 0.000 & 0.000 & 1.000 \\
\hline $\mathrm{N} 10 \mathrm{M} 302$ & 0.000 & 1.000 & 0.000 & 0.000 & 1.000 & 0.000 & 0.000 & 1.000 & 0.000 & 0.000 & 0.500 & 0.500 & 0.000 & 1.000 & 0.000 \\
\hline N10M303 & 0.330 & 0.330 & 0.330 & 0.000 & 0.860 & 0.140 & 1.000 & 0.000 & 0.000 & 0.430 & 0.290 & 0.290 & 0.000 & 0.430 & 0.570 \\
\hline N10M304 & 1.000 & 0.000 & 0.000 & 0.330 & 0.330 & 0.330 & 0.400 & 0.200 & 0.400 & 0.000 & 0.000 & 1.000 & 0.000 & 0.600 & 0.400 \\
\hline N10M305 & 0.000 & 0.000 & 1.000 & 0.350 & 0.300 & 0.350 & 0.300 & 0.250 & 0.450 & 0.000 & 0.000 & 1.000 & 0.150 & 0.450 & 0.400 \\
\hline N10M306 & 0.500 & 0.080 & 0.420 & 0.330 & 0.330 & 0.330 & 0.420 & 0.000 & 0.580 & 0.000 & 0.420 & 0.580 & 0.330 & 0.330 & 0.330 \\
\hline N10M307 & 0.310 & 0.380 & 0.310 & 0.080 & 0.460 & 0.460 & 0.460 & 0.000 & 0.540 & 0.000 & 0.000 & 1.000 & 0.330 & 0.330 & 0.330 \\
\hline N10M308 & 1.000 & 0.000 & 0.000 & 0.500 & 0.000 & 0.500 & 0.000 & 0.500 & 0.500 & 1.000 & 0.000 & 0.000 & 0.330 & 0.330 & 0.330 \\
\hline N10M309 & 0.370 & 0.370 & 0.260 & 0.260 & 0.320 & 0.420 & 0.000 & 0.000 & 1.000 & 0.550 & 0.180 & 0.260 & 0.500 & 0.160 & 0.340 \\
\hline N10M310 & 0.330 & 0.000 & 0.670 & 0.330 & 0.330 & 0.330 & 0.000 & 0.330 & 0.670 & 0.330 & 0.330 & 0.330 & 0.000 & 0.000 & 1.000 \\
\hline N10M311 & 0.330 & 0.330 & 0.330 & 0.140 & 0.430 & 0.430 & 0.430 & 0.290 & 0.290 & 0.000 & 0.000 & 1.000 & 0.330 & 0.330 & 0.330 \\
\hline N10M312 & 0.480 & 0.260 & 0.260 & 0.320 & 0.550 & 0.130 & 0.420 & 0.100 & 0.480 & 0.520 & 0.130 & 0.350 & 0.190 & 0.000 & 0.810 \\
\hline N10M313 & 0.500 & 0.250 & 0.250 & 1.000 & 0.000 & 0.000 & 0.170 & 0.170 & 0.670 & 0.420 & 0.170 & 0.420 & 0.000 & 0.000 & 1.000 \\
\hline N10M314 & 1.000 & 0.000 & 0.000 & 0.330 & 0.330 & 0.330 & 0.000 & 0.000 & 1.000 & 0.000 & 0.000 & 1.000 & 0.000 & 0.000 & 1.000 \\
\hline
\end{tabular}

\section{Table 4.9 Sample of input and output pattern pairs.}



version of the simulation sofiware package Arena (Kelton et al, 2002). This model is given in Appendix B.

Randomly selected problems from the training (data) set were tested using this Arena job shop model for all the combinations of the three dispatching rules on the 5 machines. It was seen that the simulation output from the Arena simulations matched the results from the C++ program.

Table 4.10 presents the different dispatching rules tested by the arena model for the performance objective of minimizing the makespan.

\begin{tabular}{|c|c|c|}
\hline \multicolumn{3}{|c|}{ Arena 5-machine Job shop simulation Model } \\
\hline Dispatching rules & Queue Ranking Rule & Expression/Aturibute \\
\hline SPT & Least Value First & Processing time $\left(\mathrm{P}_{\mathrm{ijk}}\right)$ \\
LPT & High Value First & Processing time $\left(\mathrm{P}_{\mathrm{ijk}}\right)$ \\
& & Remaining time \\
MWKR & High Value First & $\sum_{i=1}^{n}\left(\sum_{j . k=1}^{j, k=5}\left(\mathrm{P}_{\mathrm{ijk}}\right)-\left(\mathrm{P}_{\mathrm{i}(\mathrm{i}-1) \mathrm{k}}\right)\right.$ \\
\hline
\end{tabular}

Table 4.10 Attributes used in job shop Arena model.

\subsubsection{Data sorting}

As seen in Table 4.9, several of the output patterns show that more than one dispatching rule is favored on one or more of the machines. This arises when there exists more than one combination of dispatching rules that produce the lowest makespan. For example, in data set Nl0M306, in Table 4.9, the three output units for machine 2 show equal values of 0.330 . This indicates that all three dispatching rules appear with equal frequencies in the multiple combinations that yield minimum makespan for this problem. Using data from a problem such as this to train a network is not helpful because it introduces conflicting information and prevents the network from detecting the useful relationships between input data and optimal outputs. Therefore, if the network is trained without sorting and screening the input data, then chances are that the 
network will not learn properly and may not select the best dispatching rules. The sorting is done conveniently, using Structure Query Language (SQL).

SQL has the capabilities to sort the data considering multiple criteria at a time. In this case there are 3 outputs for each machine. There are two different sorting criteria considered:

A sorting index of 5 identifies the problem whose output units for all the five machines is either $(1,0,0),(0,1,0$,$) or (0,0,1)$. For example referring to the problem number N10M301 of Table 4.9 the output value of each machine satisfies the sorting criteria for a sorting index of 5.

A sorting index of 4 identifies the problem whose output units for any four out of the five machines is either $(1,0,0),(0,1,0$,$) or (0,0,1)$. For the remaining machine the value of the output unit is treated as zero while sorting; resulting in the sum of sorting index as 4 . For example, for the problem number N10M302 of Table 4.9 the sorting index is 4 because four out of the five machines satisfy the sorting criteria. Likewise the patterns can be sorted for sorting indices of 3,2 , 1 and 0.

The data is sorted in the descending order having the maximum sorting index (5 in this case). The sorting procedure is illustrated in Table 4.11 , after the same patterns of Table 4.9 are sorted. The first column in the target output patterns of Table 4.11 lists the sorting index for each problem; The number of outputs (equivalent to the sorting index) satisfied by the respective problems is highlighted in Table 4.11.

For all of the 7500 training problems (as explained in section 4.3.4) data are sorted simultaneously. For training the network it is not advisable to use only data whose sorting index is 5. This is because many problems exhibit multiple optimal combinations, and to help the network deal with such cases it is a good idea to incorporate some data having characteristics where more 


\begin{tabular}{|c|c|c|c|c|c|c|c|c|c|c|c|c|c|c|c|}
\hline Data Set & \multicolumn{5}{|c|}{ Total Proccssing time } & \multicolumn{5}{|c|}{ Variance of Processing time } & \multicolumn{5}{|c|}{ Mean Routing order } \\
\hline Numbers & Input 1 & Input 2 & Input 3 & Input 4 & Input 5 & Input 6 & Input 7 & Input 8 & Input 9 & Input 10 & Input 11 & Input 12 & Input 13 & Input 14 & Input 15 \\
\hline N10M301 & 0.50 & 0.71 & 0.80 & 0.93 & 1.00 & 0.75 & 0.98 & 0.51 & 1.00 & 0.46 & 0.46 & 0.60 & 0.76 & 0.60 & 0.58 \\
\hline N10M302 & 0.92 & 1.00 & 0.87 & 0.90 & 0.88 & 0.32 & 1.00 & 0.67 & 0.55 & 0.59 & $\cdot 0.48$ & 0.72 & 0.46 & 0.80 & 0.54 \\
\hline N10M314 & 0.75 & 0.35 & 1.00 & 0.67 & 0.72 & 0.64 & 1.00 & 0.58 & 0.54 & 0.42 & 0.68 & 0.36 & 0.50 & 0.58 & 0.88 \\
\hline N10M304 & 0.71 & 0.54 & 0.57 & 0.86 & 1.00 & 0.70 & 0.79 & 1.00 & 0.89 & 0.88 & 0.36 & 0.66 & 0.50 & 0.76 & 0.72 \\
\hline N10M305 & 1.00 & 0.88 & 0.63 & 0.94 & 0.82 & 0.74 & 0.97 & 1.00 & 0.60 & 0.77 & 0.32 & 0.60 & 0.60 & 0.76 & 0.72 \\
\hline N10M308 & 0.53 & 0.38 & 0.97 & 1.00 & 0.48 & 0.43 & 0.47 & 0.22 & 1.00 & 0.37 & 0.52 & 0.64 & 0.62 & 0.66 & 0.56 \\
\hline N1OM313 & 0.48 & 1.00 & 0.84 & 0.84 & 0.53 & 0.76 & 0.77 & 0.39 & 0.44 & 1.00 & 0.48 & 0.88 & 0.52 & 0.32 & 0.80 \\
\hline N1OM303 & 0.87 & 0.87 & 1.00 & 0.98 & 0.40 & 0.48 & 0.69 & 0.63 & 0.58 & 1.00 & 0.46 & 0.60 & 0.78 & 0.44 & 0.72 \\
\hline N10M307 & 0.84 & 0.74 & 1.00 & 0.55 & 0.63 & 0.88 & 0.77 & 0.84 & 0.84 & 1.00 & 0.72 & 0.68 & 0.50 & 0.54 & 0.56 \\
\hline N10M309 & 1.00 & 0.66 & 0.81 & 0.63 & 0.91 & 0.76 & 0.61 & 1.00 & 0.47 & 0.52 & 0.62 & 0.72 & 0.38 & 0.74 & 0.54 \\
\hline N10M310 & 0.52 & 0.78 & 0.95 & 1.00 & 0.63 & 0.20 & 0.68 & 0.70 & 1.00 & 0.71 & 0.68 & 0.60 & 0.64 & 0.44 & 0.64 \\
\hline N10M31I & 0.66 & 0.75 & 1.00 & 0.94 & 0.93 & 1.00 & 0.48 & 0.69 & 0.55 & 0.96 & 0.52 & 0.56 & 0.76 & 0.60 & 0.56 \\
\hline N10M306 & 0.80 & 0.95 & 0.79 & 0.57 & 1.00 & 0.88 & 0.15 & 0.51 & 1.00 & 0.64 & 0.66 & 0.54 & 0.60 & 0.54 & 0.66 \\
\hline $\mathrm{N} 10 \mathrm{M} 312$ & 0.75 & 0.66 & 0.61 & 0.96 & 1.00 & 1.00 & 0.85 & 0.72 & 0.84 & 0.77 & 0.30 & 0.76 & 0.68 & 0.68 & 0.58 \\
\hline
\end{tabular}

\begin{tabular}{|c|c|c|c|c|c|c|c|c|c|c|c|c|c|c|c|c|}
\hline \multirow{2}{*}{\begin{tabular}{|l|} 
Sorting \\
Index \\
\end{tabular}} & \multirow{2}{*}{$\begin{array}{l}\text { Data Set } \\
\text { Number }\end{array}$} & \multicolumn{3}{|c|}{ Machinel } & \multicolumn{3}{|c|}{ Machine2 } & \multicolumn{3}{|c|}{ Machine3 } & \multicolumn{3}{|c|}{ Machine4 } & \multicolumn{3}{|c|}{ Machines } \\
\hline & & o/p 1 & $\mathrm{o} / \mathrm{p} 2$ & $\mathrm{o} / \mathrm{p} 3$ & $\mathrm{o} / \mathrm{p} 4$ & $0 / p 5$ & $\mathrm{c} / \mathrm{p} 6$ & $\mathrm{o} / \mathrm{p} 7$ & $\mathrm{~d} / \mathrm{p} 8$ & $n / p 9$ & $\mathrm{~d} / \mathrm{p} 10$ & $\mathrm{~d} / \mathrm{p} 11$ & $\mathrm{a} / \mathrm{p} 12$ & $\mathrm{o} / \mathrm{p} 13$ & $n / p 14$ & $n / 21:$ \\
\hline 5 & N10M301 & 0.000 & 0.000 & 1.000 & 0.000 & 81000 & 0.000 & 0.000 & 0.000 & 84000 & 3130003 & 0.000 & 0.000 & 0.000 & 0.000 & 104000 \\
\hline 4 & $\mathrm{~N} 10 \mathrm{M} 302$ & 0.000 & $1,000^{\circ}$ & 0.000 & 0.000 & 10000 & 0.000 & 0.000 & $10000 \%$ & 0.000 & 0.000 & 0.500 & 0.500 & 0.000 & 76000 & 0.000 \\
\hline 4 & N10M314 & 11000 & 0.000 & 0.000 & 0.330 & 0.330 & 0.330 & 0,000 & 0.000 & 18000 & 0.000 & 0.000 & 1000 & 0.000 & 0.000 & $1: 000$ \\
\hline 2 & $\mathrm{~N} 10 \mathrm{M} 304$ & 1000 & 0.000 & 0.000 & 0.330 & 0.330 & 0.330 & 0.400 & 0.200 & 0.400 & 0.000 & 0.000 & $1: 000 \%$ & 0.000 & 0.600 & 0.400 \\
\hline 2 & N10M305 & 0.000 & 0.000 & 1.000 & 0.350 & 0.300 & 0.350 & 0.300 & 0.250 & 0.450 & 0.000 & 0.000 & $1000 \%$ & 0.150 & 0.450 & 0.400 \\
\hline 2 & N10M308 & 3150006 & 0.000 & 0.000 & 0.500 & 0.000 & 0.500 & 0.000 & 0.500 & 0.500 & $0000 \%$ & 0.000 & 0.000 & 0.330 & 0.330 & 0.330 \\
\hline 2 & $\mathrm{~N} 10 \mathrm{M} 313$ & 0.500 & 0.250 & 0.250 & 4100007 & 0.000 & 0.000 & 0.170 & 0.170 & 0.670 & 0.420 & 0.170 & 0.420 & 0.000 & 0.000 & 1.000 \\
\hline 1 & $\mathrm{~N} 10 \mathrm{M} 303$ & 0.330 & 0.330 & 0.330 & 0.000 & 0.860 & 0.140 & x & 0.000 & 0.000 & 0.430 & 0.290 & 0.290 & 0.000 & 0.430 & 0.570 \\
\hline 1 & N10M307 & 0.310 & 0.380 & 0.310 & 0.080 & 0.460 & 0.460 & 0.460 & 0.000 & 0.540 & 0.000 & 0.000 & 1.000 & 0.330 & 0.330 & 0.3311 \\
\hline 1 & N10M309 & 0.370 & 0.370 & 0.260 & 0.260 & 0.320 & 0.420 & 0.000 & 0.000 & m15000 & 0.550 & 0.180 & 0.260 & 0.500 & 0.160 & 0.340 \\
\hline 1 & $\mathrm{~N} 10 \mathrm{M} 310$ & 0.330 & 0.000 & 0.670 & 0.330 & 0.330 & 0.330 & 0.000 & 0.330 & 0.670 & 0.330 & 0.330 & 0.330 & 0.000 & 0.000 & F्ञ 0000 \\
\hline 1 & N10M31I & 0.330 & 0.330 & 0.330 & 0.140 & 0.430 & 0.430 & 0.430 & 0.290 & 0.290 & 0.000 & 0.000 & $1000 \%$ & 0.330 & 0.330 & 0.330 \\
\hline n & N10M306 & 0.500 & 0.080 & 0.420 & 0.330 & 0.330 & 0.330 & 0.420 & 0.000 & 0.580 & 0.000 & 0.420 & 0.580 & 0.330 & 0.330 & 0.330 \\
\hline 0 & $\mathrm{~N} 10 \mathrm{M} 312$ & 0.480 & 0.260 & 0.260 & 0.320 & 0.550 & 0.130 & 0.420 & 0.100 & 0.480 & 0.520 & 0.130 & 0.350 & 0.190 & 0.000 & $0.811^{\circ}$ \\
\hline
\end{tabular}

Table 4.11 Input and output patterns of Table 4.9 after sorting. 
than one dispatching rules is favored by one of the machines. This will help the network to learn the stronger relationships between input and output patterns. On the other hand, using patterns with lower sorting indices could hamper the learning. Therefore, it was decided to incorporate only those patterns with sorting indices 4 and above for the training. Thus, ignoring all the data having a sorting index of less than 4 , the result is a 2494 training data set extracted from the 7500 original problems for training the neural network.

\subsection{Design of Neural Network}

Once the pre-processing (sorting) of the data is done, the next step is to train the neural network. However, the final number of units for the hidden layer still needs to be decided.

\subsubsection{Hidden Layer}

The hidden layer is mainly required to overcome the non-linear learning problem. It is also where the network learns interdependencies in the model. Figure 4.3 provides some detail into what goes on inside a hidden node. Neurons of the hidden layer receive inputs from neurons of the input layer, depending on the sum of the input weights that produce an output. The output is then further transferred to units of the output layer.

Garson, (1998) states that "if the problem has a linear solution then it may not be appropriate to use hidden layers. In theory, a neural network with at least one layer and adequate number of hidden units is capable of solving most problems. However, in practice one or more layers may be used depending on the complexity of a problem (such 


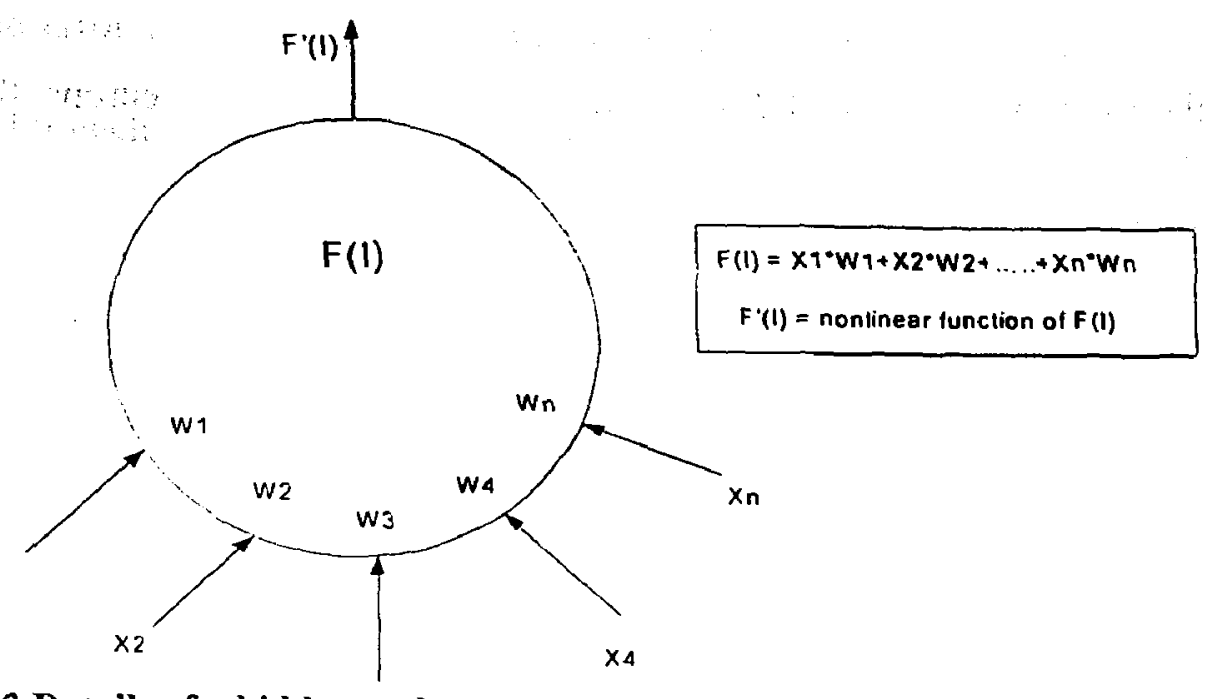

Fig. 4.3 Details of a hidden node

as complicated function fitting problems) and cost and resources. As the number of hidden layers increase, the meaningfulness of the back propagated error term decreases. Moreover, the training time increases by an order of the magnitude for each additiona] hidden layer." Thus, considering all the above-mentioned factors, only one hidden layer is considered for building the neural network.

\subsubsection{Hidden Units (neurons)}

In most situations, there is no magic formula that finds the best number of hidden units without training several networks and estimating the generalization error of each. If only a few hidden units are considered, then the network may not train well because the model lacks sufficient complexity to reflect input-output patterns in the training set. On the other hand, if too many hidden units are used, then the network may overtrain and generalization will suffer as the network simply memorizes the input-output patterns in the training set.

Some researchers have developed a heuristic approach to determine the best number of hidden neurons, (Smith, 1999). Two examples of these are the following: 
- If the input patterns are of dimension $\mathrm{N}$ and the output of $\mathrm{K}$ neurons, then the number of hidden layers would be $J=\sqrt{N x K}$ neurons.

- Another approximation is $J=\frac{1}{2}(N+K)+\sqrt{P}$, where $\mathrm{P}$ is the number of pattems in the training set. This is the default formula used in the commercial neural network package Neuroshell 2.

- On the other hand (Baily and Thompson, (1990)) suggest that the number of hidden neurons in a three layer neural network, which include input, output and hidden layers should be $75 \%$ of the number of neurons in the input layer.

Most of the above heuristics are based on the assumptions that the training set is at least twice as large as the number of weights and preferably at least four or more times larger than the number of weights. If the same is not the case, then the number of hidden neurons will be affected by the number of training sets. Moreover, there are some other factors like the amount of noise in the test patterns, the complexity of the function or classification to be learned, the architecture, etc. that will also have impact on the number of hidden neurons. A trial and error approach is often taken starting with a modest number of hidden neurons and gradually increasing this number until the network fails to reduce its error Kaastra and Boyd, (1996) and Garson, (1999). This last approach is the one adopted in this study for determining the number of units.

\subsubsection{Training Stopping Criteria}

A neural network is trained in epochs, where each epoch represents a complete pass of the training set through the network. When each training pattern is presented to the 
network, the error between the actual outputs in the training pattern and the network's predictions for each of the network's outputs is computed. The total error for each pattern is the sum of the squares of the differences. At the end of each epoch the average error over all training patterns is computed. As the epochs progress and the training continues, the network learning improves, i.e.. the error on the training and test sets decreases. For the test set, however, there comes a point where the error in the test examples starts to increase wjth additional training. While training a neural network, one expects to obtain a network with optimal generalization performance. Thus, the question of when to end the training is a critical one. There are different choices for stopping criteria for both training and testing sets adopted from Neuroshell 2, which are based on:

- The average error in the training set. End training when the lowest value for the average error in the training set is reached.

- The largest average error in the training set. This is the network's latest computation for the difference between the network's predictions and the actual predictions for data in the training set.

- The events since the minimum average error in the testing set. An event is the presentation of a single training pattern to the neural network.

Additionally, Neuroshell 2 offers two options about automatic saving of the training based on:

- Best training set saves the network every time it reaches a new minimum average error for the training set.

- Best testing set saves the network every time it reaches a new minimum average error for the test set. 


\subsubsection{Training Procedure}

The following steps are used in order to achieve a generalization capability in the performance of a trained neural network.

- Step I: Train the network with the training patterns and monitor the minimum average error. Once a new minimum average error is observed, then interrupt the training. Save the neural weights and go to step 2.

- Step 2: Apply the partially trained network to the test set and compute the performance measure (using the $\mathrm{C}++$ program of Appendix $\mathrm{A} 3$ ). If the performance measure (either total makespan or total mean flowtime) is improved upon from the previous trial, then return to step 1 . Otherwise proceed to step 3.

- Step 3: Save the current weights as a final trained network.

\subsubsection{To Find optimal number of Hidden neurons (units)}

In order to find the best number of hidden neurons, a trial and error approach is carried out involving 6 to 18 hidden neurons using the above-mentioned training stopping criteria. The smallest number represents too few and the highest number too many hidden neurons. The training trials were done using the commercial software "Neuroshell 2", and with the parameters listed in Appendix $\mathrm{C} 1$.

A new set of 1200 randomly generated test problems (having random seeds different than the training data set) were used. The original 2494 training patterns (as explained in the section 4.3.6) are considered for training. The objective is to find the size of the hidden layer that minimizes the obtained makespan for the test set (refer step 2 of the training procedure). 
Once the training is completed the test job shop problems are run (using the $\mathrm{C}++$ program of Appendix A3) to see how accurately the neural network has assigned the priority rules. The neural network's final performance for each trial with a different number of hidden neurons is tabulated in Table 4.12, which expresses the BPNN results in terms of the total makespans of the test set problems. This is further illustrated graphically in Figure 4.4.

\begin{tabular}{|c|c|c|c|}
\hline $\begin{array}{c}\text { Experiment } \\
\text { Number }\end{array}$ & $\begin{array}{c}\text { Number of } \\
\text { Hidden Neurons }\end{array}$ & $\begin{array}{c}\text { Total } \\
\text { Makespan }\end{array}$ & $\begin{array}{c}\text { Minimum } \\
\text { Average Error }\end{array}$ \\
\hline 1 & 6 & 1398179 & 1.447 \\
2 & 8 & 1394552 & 1.456 \\
3 & 9 & 1393378 & 1.437 \\
4 & 10 & 1395058 & 1.439 \\
5 & 15 & 1397199 & 1.457 \\
6 & 18 & 1397533 & 1.482 \\
\hline
\end{tabular}

Table 4.12 Total makespan using BPNN with various number of hidden neurons.

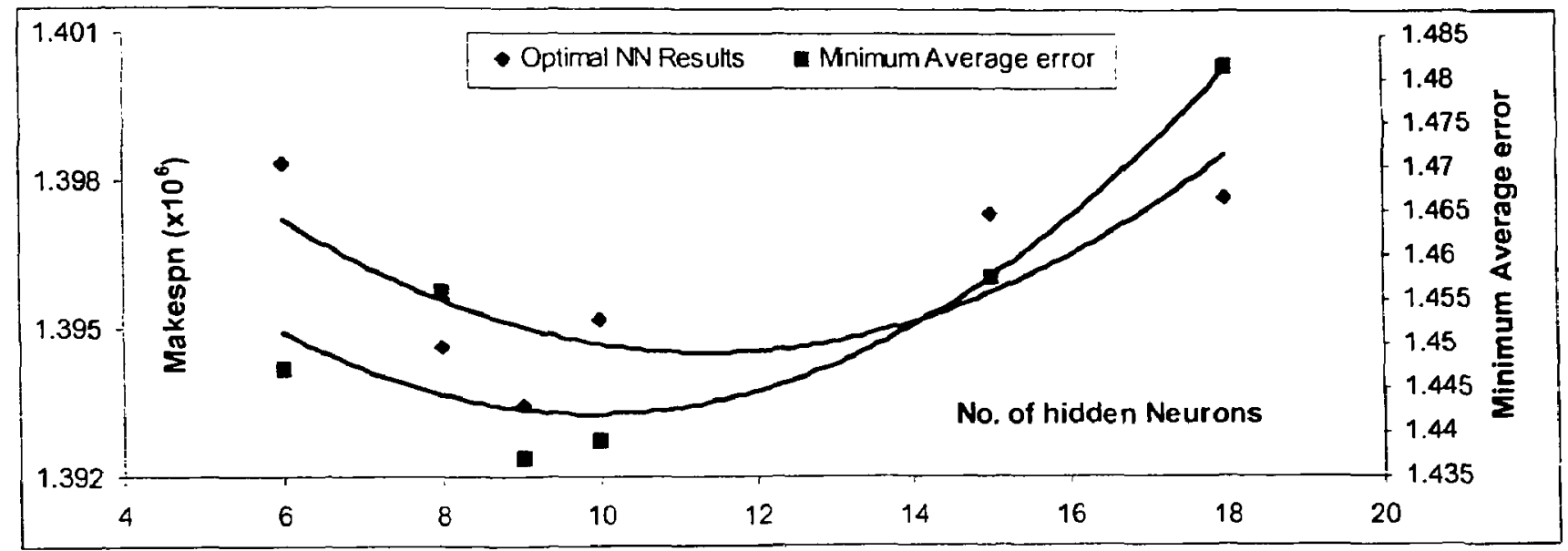

Fig.4.4 Effect of number of hidden neuron on the minimum average error for makespan.

When the number of hidden neurons is small, the corresponding minimum average error (see section 4.4.3) is also low. However, the BPNN results are not the best. Figure 4.3 shows that the BPNN performs best, and the minimum average error is lowest when the number of hidden neurons is 9 . With a higher number of hidden neurons, the network appears to memorize rather than learn the patterns. So, the chosen network has a 15-9-15 structure. The next section explains in greater detail the training of the 15-9-15 network. 
It is noteworthy that the same training process, training data and parameters described in the next section were also used in the trials to determine the number of hidden neurons, presented in Table 4.12.

\subsection{Training the BPNN}

A total of 2494 training patterns extracted from problem instances combining $n=10$, 15,20 for the five machines is considered for training the network. Similarly, a test data set of 1200 problems with the same combinations of $n$ as the training set is generated separately. As explained in the preceding chapter, the default values of Neuroshell 2 for training are a learning rate $=0.1$, momentum $=0.1$ and initial weights $=0.3$. The training was performed on AMD $900 \mathrm{MHz}$ Presario personal computer. The training is carried out and the total of the makespans for the test set is recorded for every epoch that produces further reduction in the minimum average error (see section 4.4.3). The training results are tabulated as shown in Table 4.13. The training procedure is extended from generalization test number 9 to 16 (refer to the first column of Table 4.13) in order to observe the behavior of the neural network on the test set, if the training is continued beyond a certain point. In other words, to observe what happens when the neural network is over trained.

Figure 4.5 presents the behavior of the minimum average error on the training set and the corresponding neural network results on the test set. It can be seen that as the minimum average error is reduced, the BPNN results improve correspondingly up to a certain point (generalization test number 8 in this case), beyond which the results start 
deteriorating. $\mathrm{lt}$ is at this point that the network generalizes best, and no further improvement is possible with continued training.

\begin{tabular}{|c|c|c|c|c|c|}
\hline $\begin{array}{c}\text { Generalization } \\
\text { Test }\end{array}$ & $\begin{array}{c}\text { Minimum } \\
\text { Average Error }\end{array}$ & Epoch & $\begin{array}{c}\text { Learning } \\
\text { Event }\end{array}$ & $\begin{array}{c}\text { Time } \\
(\mathrm{Sec})\end{array}$ & $\begin{array}{c}\text { BPNN } \\
\text { Suggested } \\
\text { DR }\end{array}$ \\
\hline 1 & 1.5517 & 2 & 4988 & 3 & 1449074 \\
2 & 1.5295 & 5 & 12470 & 8 & 1439644 \\
3 & 1.4938 & 12 & 29928 & 17 & 1414140 \\
4 & 1.4906 & 14 & 34916 & 19 & 1410225 \\
5 & 1.4760 & 18 & 44892 & 25 & 1409133 \\
6 & 1.4711 & 21 & 52374 & 30 & 1400621 \\
7 & 1.4558 & 23 & 57362 & 33 & 1403802 \\
8 & 1.4372 & 38 & 92278 & 52 & 1393378 \\
9 & 1.4358 & 46 & 114724 & 65 & 1398482 \\
10 & 1.4336 & 50 & 124700 & 70 & 1399688 \\
11 & 1.4238 & 75 & 187050 & 105 & 1398570 \\
12 & 1.4196 & 80 & 199520 & 113 & 1400944 \\
13 & 1.4051 & 148 & 369112 & 213 & 1401905 \\
14 & 1.3890 & 214 & 533716 & 315 & 1405274 \\
15 & 1.3857 & 407 & 1015058 & 583 & 1400672 \\
16 & 1.3776 & 642 & 1601148 & 1002 & 1402333 \\
\hline
\end{tabular}

Table 4.13 Training and generalization results for minimizing makespan.

\subsection{Implementation}

Once the training of the proposed network is completed, the next step is to see how well the neural network performs in selecting dispatching rules. In order to explain how the neural network assigns a dispatching rule to each machine, an example of 20 jobs on 5 machines is given in Table 4.14. The above example data are converted to a neural network input representation by following the procedure explained in section 4.3.2. The resulting 15-unit input vector is given in Table 4.15. 


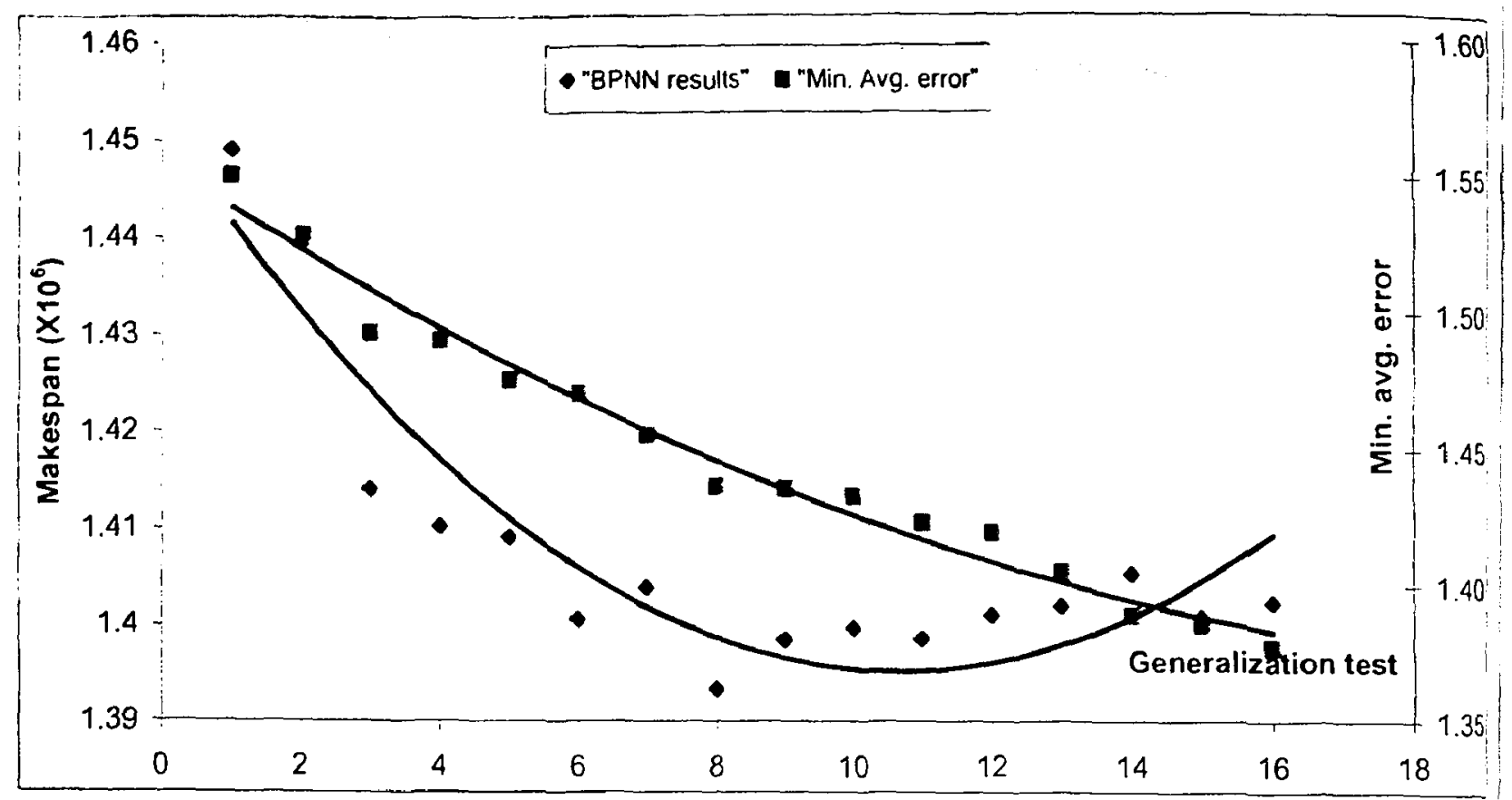

Fig. 4.5 BPNN generalization $\mathrm{V} / \mathrm{S}$ minimum average error for makespan.

\begin{tabular}{|c|ccccc|ccccc|}
\hline $\begin{array}{c}\text { Job } \\
\text { no }\end{array}$ & \multicolumn{5}{|c|}{ Routes } & \multicolumn{5}{|c|}{ Processing time } \\
\hline 1 & 2 & 1 & 4 & 3 & 5 & 52 & 60 & 94 & 33 & 23 \\
2 & 2 & 1 & 5 & 3 & 4 & 58 & 41 & 20 & 34 & 57 \\
3 & 5 & 4 & 3 & 1 & 2 & 51 & 77 & 33 & 74 & 37 \\
4 & 5 & 2 & 4 & 3 & 1 & 46 & 34 & 92 & 62 & 53 \\
5 & 5 & 4 & 3 & 1 & 2 & 46 & 92 & 58 & 63 & 35 \\
6 & 5 & 2 & 4 & 3 & 1 & 37 & 66 & 61 & 29 & 55 \\
7 & 5 & 2 & 4 & 3 & 1 & 53 & 68 & 90 & 34 & 63 \\
8 & 5 & 4 & 3 & 1 & 2 & 28 & 84 & 45 & 70 & 46 \\
9 & 1 & 4 & 3 & 5 & 2 & 68 & 87 & 42 & 49 & 32 \\
10 & 5 & 2 & 4 & 3 & 1 & 22 & 53 & 92 & 46 & 50 \\
11 & 2 & 1 & 4 & 3 & 5 & 61 & 67 & 64 & 24 & 53 \\
12 & 5 & 2 & 4 & 3 & 1 & 26 & 57 & 63 & 32 & 76 \\
13 & 3 & 1 & 4 & 2 & 5 & 41 & 68 & 65 & 67 & 38 \\
14 & 2 & 1 & 4 & 3 & 5 & 36 & 37 & 95 & 47 & 45 \\
15 & 5 & 2 & 4 & 3 & 1 & 40 & 66 & 67 & 30 & 71 \\
16 & 2 & 1 & 5 & 3 & 4 & 61 & 40 & 15 & 58 & 69 \\
17 & 3 & 1 & 4 & 2 & 5 & 55 & 61 & 91 & 59 & 14 \\
18 & 1 & 4 & 3 & 5 & 2 & 65 & 84 & 52 & 38 & 67 \\
19 & 1 & 4 & 3 & 5 & 2 & 42 & 82 & 26 & 16 & 32 \\
20 & 2 & 1 & 4 & 3 & 5 & 51 & 45 & 80 & 45 & 51 \\
\hline
\end{tabular}

Table 4.14 A 20-job example problem. 


\begin{tabular}{|c|c|c|c|c|c|c|c|c|c|c|c|c|c|c|c|}
\hline Set No. & \multicolumn{5}{|c|}{ Total Processing time } & \multicolumn{5}{|c|}{ Variance of Proces } & \multicolumn{5}{|c|}{ Mean routing order } \\
\hline Input & 1 & 2 & 3 & 4 & 5 & 6 & 7 & 8 & 9 & 10 & 11 & 12 & 13 & 14 & 15 \\
\hline 20E] & 0.74 & 0.65 & 0.52 & 1.0 & 0.4 .5 & 0.792 & 0.91 & 0.69 & 0.86 & 1 & 0.61 & 0.56 & 0.68 & 0.58 & 0.57 \\
\hline
\end{tabular}

Table 4.15 Input vector for example problem of Table 4.14 .

A trained network generates output in response to the data vector presented to it at the input layer. When the input vector of Table 4.15 is introduced to the BPNN, which has been just been trained for the minimization of makespan, a feed forward set of computations produces the output vector given in Table 4.16.

\begin{tabular}{|c|c|c|c|c|c|c|c|c|c|c|c|c|c|c|c|}
\hline \multirow{3}{*}{ Jutput } & \multicolumn{3}{|c|}{ Machine } & \multicolumn{3}{|c|}{ Machine2 } & \multicolumn{3}{|c|}{ Machine 3} & \multicolumn{3}{|c|}{ Machine4 } & \multicolumn{3}{|c|}{ Machine5 } \\
\hline & 1 & 2 & 3 & 4 & 5 & 6 & 7 & 8 & 9 & 10 & 11 & 12 & 13 & 14 & 15 \\
\hline & SPT & LPT & MWKR & SPT & LPT & MWKR & SPT & LPT & MWKR & SPT & LPT & MWKR & SPT & LPT & MWKR \\
\hline$N N$ & 0.332 & 0.206 & 0.449 & 0.462 & 0.117 & 0.411 & 0.296 & 0.394 & 0.266 & 0.259 & 0.062 & 0.694 & 0.507 & 0.228 & 0.229 \\
\hline
\end{tabular}

Table 4.16 BPNN output for an example problem of Table 4.14.

The maximum value in each set of 3 units associated with each machine is identified. The dispatching rule corresponding to those units (highlighted in the Table 4.16) is then assigned to the machines. In the above example, the neural network's choice for the first machine is MWKR; the second machine is SPT; the third machine is LPT; the fourth machine is MWKR; and the last machine is SPT. Applying this allocation of dispatching rules, the makespan for the problem is found to be 1652 . This compares to a minimum makespan of 1628 for the optimal rule combination of SPT-SPT-LPT-MWKRSPT applied on machines 1 to 5 respectively. The neural network's suggested combination of dispatching rules deviates from the optimal combination only for the first machine, where instead of SPT, the BPNN applies MWKR. Moreover, if all the machines use only a single rule for all machines (either SPT, LPT or MWKR), then the resulting makespans are 1720,1699 , and 1651 respectively. 


\subsection{BPNN model for minimizing mean flowtime criteria}

For the performance criterion of minimizing the mean flowtime, a BPNN model is constructed by the same methodology as used for minimizing makespan. The only difference however, lies in the 3 dispatching rules, where SPT, PT+WINQ and LWKR are considered for the flowtime objective. This is because past research shows that all these rules are cffective in minimizing mean flowtime (Waikar et al, 1995 and Rajender and Holthaus, 1999).

In order to find an optimal number of hidden neurons, some modification was done in the step used for the makespan BPNN. A total of 2636 training patterns were extracted from problems of $n=10,15$ and 20 . The same 1200 test data set used for makespan was also used in the flowtime case. The NN parameters used for finding optimal number of hidden neurons is given in Appendix C2.

Table 4.17 represents neural network results for seven different numbers of hidden neurons and Figure 4.6 depicts the effect of the number of different hidden neurons on the neural network and the minimum average error. Hence, the network used for minimizing the mean flowtime has a $15-20-15$ structure.

\begin{tabular}{|c|c|c|c|}
\hline $\begin{array}{c}\text { Experiment } \\
\text { Number }\end{array}$ & $\begin{array}{c}\text { Number of } \\
\text { Hidden Neurons }\end{array}$ & $\begin{array}{c}\text { Total Mean } \\
\text { Flowrime }\end{array}$ & $\begin{array}{c}\text { Minimum } \\
\text { Average Error }\end{array}$ \\
\hline 1 & 10 & 873181.62 & 1.700 \\
2 & 15 & 872529.56 & 1.671 \\
3 & 17 & 872254.31 & 1.668 \\
4 & 18 & 871955 & 1.641 \\
5 & 19 & 871687.81 & 1.659 \\
6 & 20 & 871669.31 & 1.594 \\
7 & 25 & 871967.44 & 1.655 \\
\hline
\end{tabular}

Table 4.17 Total mean flowtimes for BPNN with various numbers of hidden neurons. 


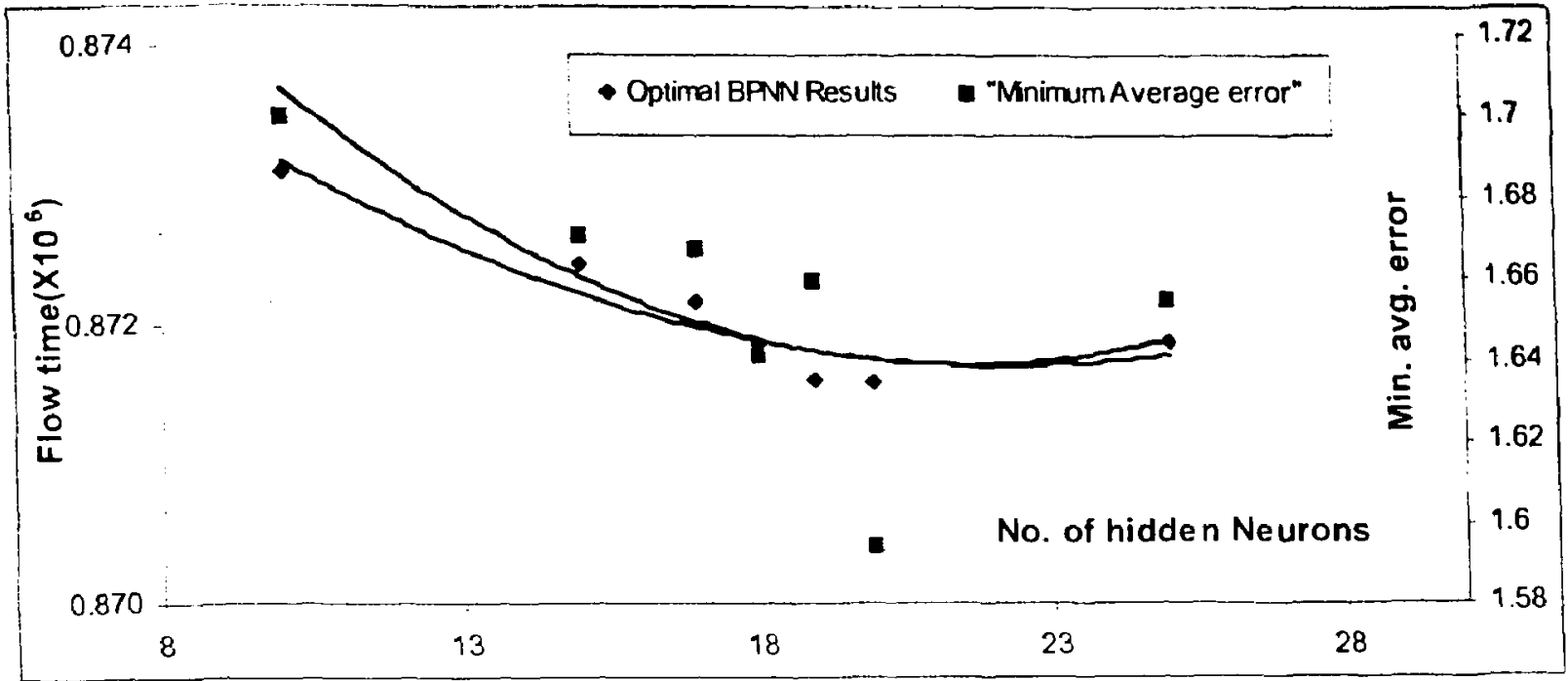

Fig. 4.6 Effect of number of hidden neurons on the minimum average error for mean flowtime.

In order illustrate how the trained network selects dispatching rules to minimize flowtime, the same example problem of Table 4.14 is considered. The input vector is the same; however, the output vector represents different rules. Output resulting from the feed forward processing induced by the application of input vector of table 3.16 to the BPNN (15-20-15) trained for flowtime minimization is given in Table 4.18.

\begin{tabular}{|c|c|c|c|c|c|c|c|c|c|c|c|c|c|c|c|}
\hline Output & \multicolumn{3}{|c|}{ Machine 1} & \multicolumn{3}{|c|}{ Machine2 } & \multicolumn{3}{|c|}{ Machine 3} & \multicolumn{3}{|c|}{ Machine4 } & \multicolumn{3}{|c|}{ Machines } \\
\hline$\overline{\mathrm{DR}}$ & SPT & $\begin{array}{l}\text { WINQ } \\
+\mathrm{PT}\end{array}$ & LWKR & SPT & $\begin{array}{l}\text { WINQ } \\
+\mathrm{PT} \\
\end{array}$ & LWKR & SPT & $\begin{array}{l}\text { WINQ } \\
+\mathrm{PT} \\
\end{array}$ & LWKR & SPT & $\begin{array}{c}\text { WINQ } \\
+\mathrm{PT} \\
\end{array}$ & LWKR & SPT & $\begin{array}{l}\text { WINQ } \\
+\mathrm{PT}\end{array}$ & LWKR \\
\hline Output & 0.167 & 0.070 & 0.737 & 0.014 & 0.150 & 0.776 & 0.216 & 0.239 & 0.556 & 0.956 & 0.0 & $0.04]$ & 0.07 & 0.228 & 0.708 \\
\hline
\end{tabular}

Table 4.18 Mean flowtime BPNN optimal example problem of Table 4.14.

The above neural network's recommendations (highlighted in the Table 4.18) are LWKR for the first, second and third machines, SPT for the fourth machine and LWKR for the last machine. This combination results in a mean flowtime of 936.65 , which " compares to a possible minimum of 921.45 . The neural network's suggested combination of dispatching rule deviates from the optimal combination only for the last machine, where instead of WINQ+PT, the BPNN suggests LWKR. On the other hand, if all the machines applied only one of SPT. WINQ+PT or LWKR dispatching rules on all the machines, then the mean flowtimes are $989.95,1031.55$, and 952.85 respectively. 
In this chapter, a detailed description of the design of BPNN for two different performance criteria is described. For the makespan, a BPNN model of 15-9-15 was designed and for the mean flowtime a BPNN model of 15-20-15. For both the performance criteria, the trained network suggested a combination of dispatching rules that were reasonably close to the optimal combinations. The next chapter will test the generalization capabilities of both trained networks for problems with the number of jobs ranging between a minimum of 10 to a maximum of 100 jobs. 


\section{CHAPTER 5}

\section{Analysis and Discussion}

In this chapter, the generalization capability of the BP.VN and its effectiveness in providing results to new problems (unseen during the neural network's training) will be examined for the performance objectives of minimizing makespan and mean flowtime. This will be followed by a discussion on the results and an analysis of variance.

\subsection{Analysis of Trained Neural Network}

Once the training of the neural network as explained in the previous chapter is completed, it is necessary to test how well the neural network's suggested combination of dispatching rules perform in new problems.

\subsubsection{Neural Network Weights}

The trained neural network holds its knowledge in the weights between the nodes. The final weights from the training process using Neuroshell 2 (as explained in the section 4.4.4) are provided in Appendix D1 and D2 for the makespan and mean flowtime networks respectively.

\subsection{Test Problems}

A total of fourteen different sets of new problems with the job numbers (n) ranging from 10 to 100 jobs is created using the same parameters used for the testing sets in the 
previous chapler. Each set contains fifty test problems generated randomly using different random seeds for each different category of $\mathrm{n}$. Altogether, 700 problems are generated using the $\mathrm{C}$ program of Appendix A4.

\subsection{Test Results for Minimizing Makespan}

Once the generation of the various problems is completed as mentioned above, the next step is to obtain and compare the total makespan in each set. Each set of fifty problems is tested 4 times. In the first trial, the SPT rule is applied on all five machines and the total makespan is recorded. In the second and third trials, the LPT and MWKR rules are tested respectively, in a similar fashion. Finally, the BPNN is used in the fourth trial to select dispatching rule combinations for the test problems. The BPNN generates the preferred dispatching rule for each machine by feed forward processing identical to that described for the example problem in section 4.7.

Table 5.1 presents the total of the makespans in each of the trials for all 14 sets of test problems. In order to evaluate how well the neural network's (BPNN) suggested combination of dispatching rules performs, a comparison with the makespans produced by optimal combinations of the dispatching rules is also presented in Table 5.1.

As can be seen from the results of Table 5.1, the trained neural network generates better total makespan results as compared to using the same dispatching rule on all the machines. Furthermore, the percentage deviation of the neural network (BPNN) results from the optimal is close.

Deviation is calculated by using equation 5.1. The deviation of BPNN from optimal results ranged from a minimum of $0.3 \%$ to a maximum of $3.0 \%$. 


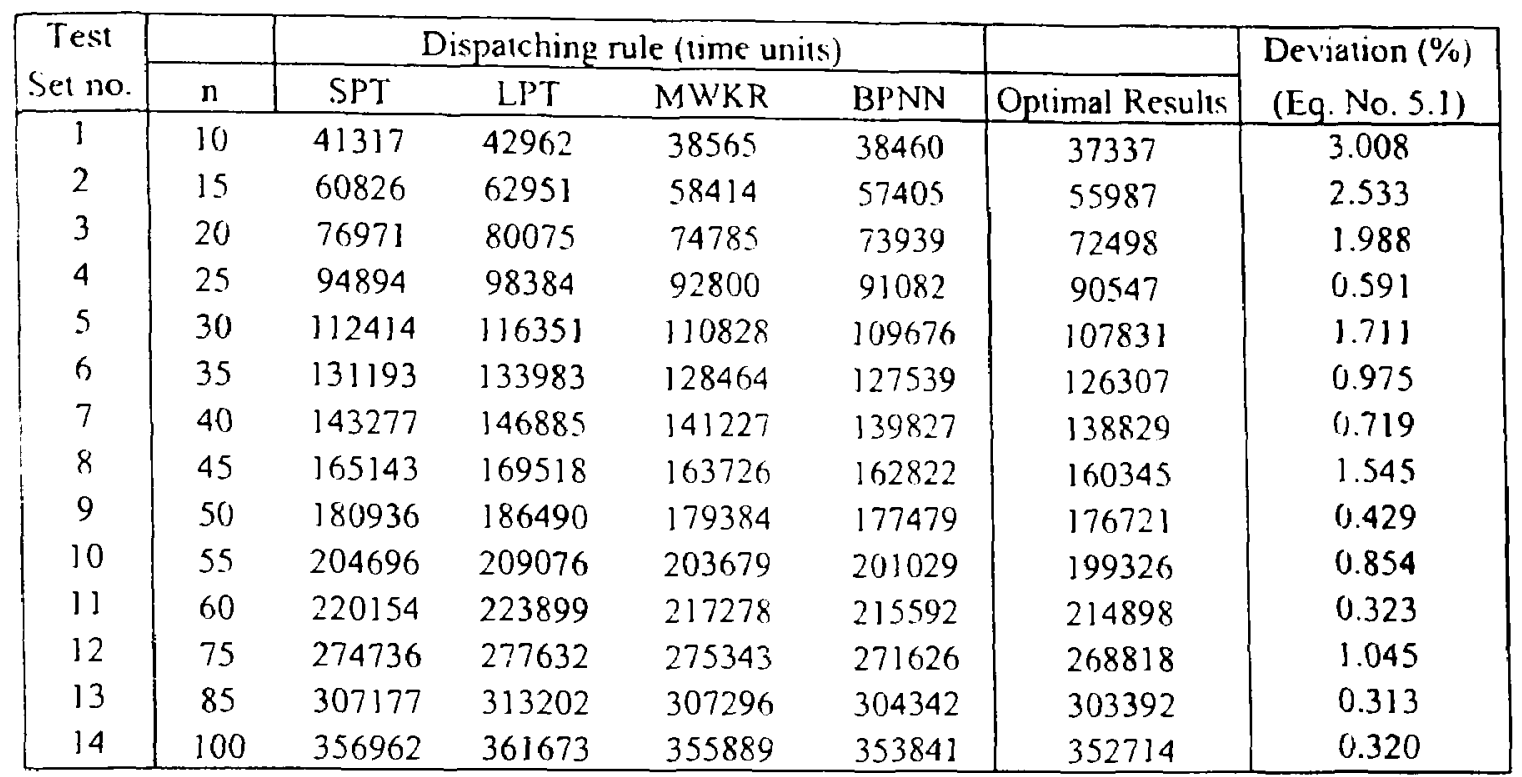

Table 5.1 Summary of total makespan in sets of 50 test problem for various $n$.

$$
\text { Deviation }(\%)=\frac{(B P N N-O P T I M A L)}{\text { OPTIMAL }} * 100
$$

As a sample, results for all the 50 problems of test set no. $9(n=50)$ are presented in Table 5.2. These results show that there is not much difference between the neural network's performance and the optimal combination of dispatching rules, as further illustrated in Figure 5.1. The neural network achieved optimal results in $80 \%$ of the test problems and deviated by an average of $2.1 \%$ from the optimal in the rest. For comparison SPT, LPT and MWKR matched the optimal result in $14 \%, 35 \%$ and $4 \%$ respectively of the test problems. Similar comparisons between optimal and BPNN results for individual problems in the other thirteen sets are attached in the Appendix El.

\subsection{Test Results for Minimizing Mean Flowtime}

The same sets of test problems used for testing the makespan are also used for testing the generalization capability and the effectiveness of the neural network that has been trained to minimize mean flowtime. This neural network is tested in the same manner as the previous network used for testing makespan. In the first three trials each 


\begin{tabular}{|c|c|c|c|c|c|c|c|c|c|c|c|}
\hline \multirow{2}{*}{$\begin{array}{c}\text { Data set } \\
\text { No. }\end{array}$} & \multicolumn{4}{|c|}{ Disparching rule (lime units) } & \multirow[b]{2}{*}{ Optimal } & \multirow{2}{*}{$\begin{array}{c}\text { Data sel } \\
\text { No. }\end{array}$} & \multicolumn{4}{|c|}{ Dispatching rule (time units) } & \multirow[b]{2}{*}{ Optimal } \\
\hline & SP] & 1.PT & MWKR & BPNN & & & SPT & LPT & MWKR & $\mathrm{BPNN}$ & \\
\hline $50 \mathrm{NI}$ & 2087 & 2181 & 2087 & 2087 & 2087 & $50 \mathrm{~N} 26$ & 3017 & 3224 & 3190 & 2993 & 2993 \\
\hline $50 \mathrm{~N} 2$ & 3358 & 3379 & 3355 & 3239 & 3234 & $50 \mathrm{~N} 27$ & 3971 & 3999 & 3881 & 3881 & 3881 \\
\hline $50 \mathrm{~N} 3$ & 2517 & 2669 & 2449 & 2449 & 2449 & $50 \mathrm{~N} 28$ & 3406 & 3322 & 3189 & 3189 & 3189 \\
\hline $50 \mathrm{~N} 4$ & 3520 & 3571 & 3508 & 3508 & 3508 & $50 \mathrm{~N} 29$ & 3654 & 3614 & 3540 & 3540 & 3540 \\
\hline $50 \mathrm{~N} 5$ & 3719 & 3774 & 3677 & 3677 & 3677 & $50 \mathrm{~N} 30$ & 4119 & 4154 & 3986 & 3986 & 3986 \\
\hline 50N6 & 3805 & 3754 & 37.54 & 3754 & 3754 & $50 \mathrm{~N} 31$ & 3548 & 3648 & 3503 & 3503 & 3503 \\
\hline 50N7 & 3427 & 3569 & 340() & 3406 & 3406 & $50 \mathrm{~N} 32$ & 3911 & 4014 & 3874 & 3874 & 3874 \\
\hline $50 \mathrm{~N} 8$ & 3748 & 3769 & 3620 & 3620 & 3620 & $50 \mathrm{~N} 33$ & 3632 & 3629 & 3482 & 3482 & 3482 \\
\hline 50N9 & 4349 & 4743 & 4134 & 4134 & 4134 & $50 \mathrm{~N} 34$ & 3821 & 4106 & 4035 & 3920 & 3689 \\
\hline $50 \mathrm{~N} 10$ & 3954 & 3957 & 3856 & 3856 & 3856 & $50 \mathrm{~N} 35$ & 3887 & 3994 & 3885 & 3885 & 3856 \\
\hline $50 \mathrm{~N} 11$ & 3759 & 3887 & 3739 & 3584 & 3568 & $50 \mathrm{~N} 36$ & 3324 & 3351 & 3221 & 3183 & 3183 \\
\hline $50 \mathrm{~N} 12$ & 3144 & 3360 & 3143 & 3143 & 3055 & $50 \mathrm{~N} 37$ & 3946 & 3982 & 3774 & 3774 & 3774 \\
\hline $50 \mathrm{~N} 13$ & 3138 & 3321 & 3104 & 3104 & 3104 & $50 \mathrm{~N} 38$ & 3317 & 3551 & 3325 & 3287 & 3287 \\
\hline $50 \mathrm{~N} 14$ & 3993 & 4043 & 4001 & 4001 & 3993 & 50 N39 & 4049 & 4159 & 3946 & 3946 & 3946 \\
\hline $50 \mathrm{~N} 15$ & 3814 & 4025 & 4115 & 3865 & 3645 & $50 \mathrm{~N} 40$ & 3849 & 3994 & 3845 & 3845 & 3845 \\
\hline $50 \mathrm{~N} 16$ & 3952 & 4152 & 4002 & 3952 & 3952 & $50 \mathrm{~N} 41$ & 3433 & 3562 & 3515 & 3462 & 3433 \\
\hline $50 \mathrm{~N} 17$ & 3546 & 3919 & 3635 & 3546 & 3546 & $50 \mathrm{~N} 42$ & 3688 & 3799 & 3683 & 3683 & 3683 \\
\hline $50 N 18$ & 4029 & 4214 & 3835 & 3835 & 3835 & $50 \mathrm{~N} 43$ & 3740 & 3828 & 3590 & 3590 & 3590 \\
\hline $50 \mathrm{~N} 19$ & 3612 & 3826 & 3572 & 3572 & 3572 & $50 \mathrm{~N} 44$ & 3720 & 3814 & 3960 & 3699 & 3601 \\
\hline $50 \mathrm{~N} 20$ & 3248 & 3396 & 3163 & 3163 & 3163 & $50 \mathrm{~N} 45$ & 3940 & 3917 & 3798 & 3798 & 3798 \\
\hline $50 \mathrm{~N} 21$ & 3713 & 3705 & 3566 & 3566 & 3566 & 50N46 & 3516 & 3408 & 3408 & 3408 & 3408 \\
\hline $50 \mathrm{~N} 22$ & 3534 & 3626 & 3409 & 3409 & 3409 & $50 \mathrm{~N} 47$ & 3331 & 3379 & 3263 & 3263 & 3263 \\
\hline $50 \mathrm{~N} 23$ & 3451 & 3558 & $345]$ & 3451 & 3451 & 50N48 & 3862 & 3876 & 3719 & 3719 & 3719 \\
\hline $50 \mathrm{~N} 24$ & 3751 & 4017 & 3743 & 3743 & 3743 & 50N49 & 3313 & 3419 & 3156 & 3156 & 3156 \\
\hline $50 \mathrm{~N} 25$ & 4037 & 4080 & 3978 & 3978 & 3978 & $50 \mathrm{~N} 50$ & $\begin{array}{c}3737 \\
180036\end{array}$ & 4252 & 4314 & 3771 & 3737 \\
\hline & & & & & & Total & 180936 & 186490 & 179384 & 177479 & 176721 \\
\hline
\end{tabular}

Table 5.2 Results for individual problems for $n=50$ (set no. 9 in Table 5.1).

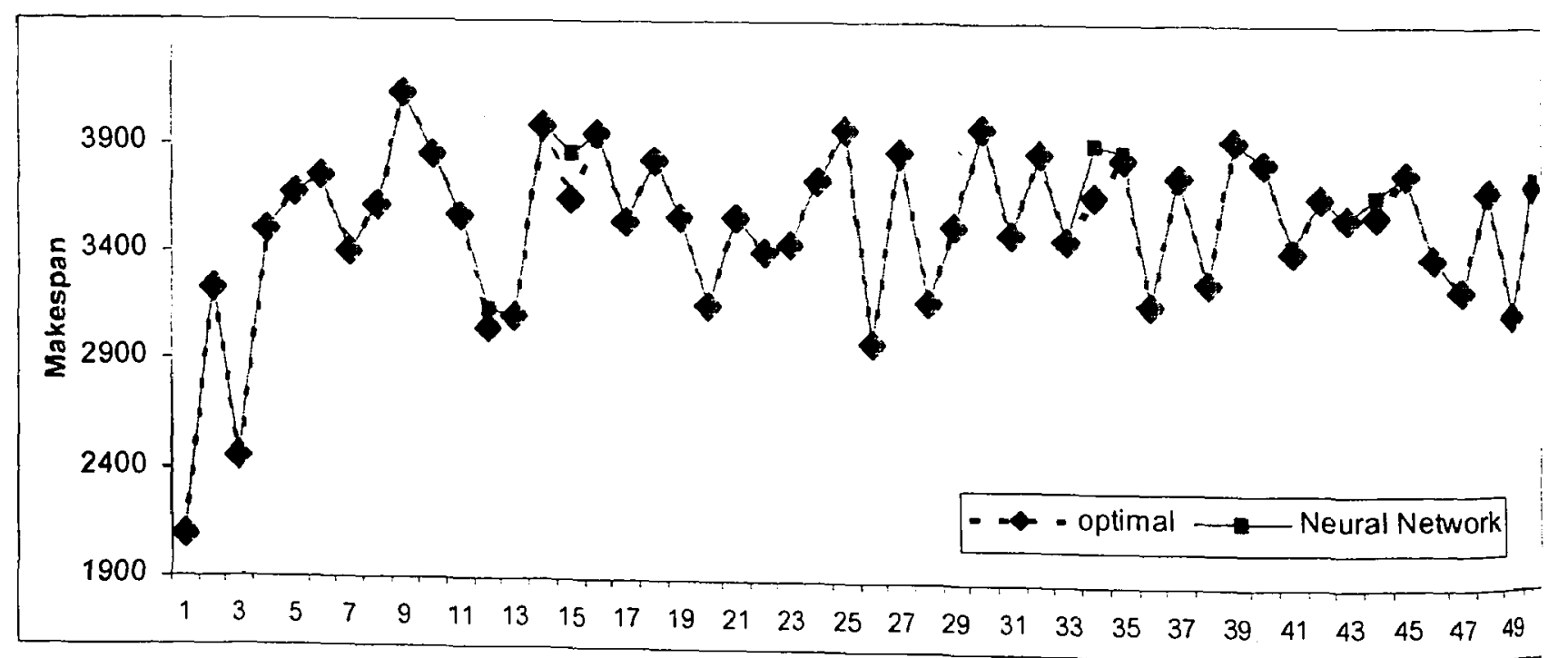

Fig 5.1 BPNN makespan compared to makespans from optimal rule combinations for $n=50$. 
set is tested using SPT, WINQ+PT and LWKR respectively on all machines. BPNN is tested in the fourth trial. The results (expressed as total of mean flowtime) are given in Table 5.3 .

\begin{tabular}{|c|c|c|c|c|c|c|c|}
\hline Test & & \multicolumn{4}{|c|}{ Dispatching rule (time units) } & & Deviation (\%) \\
\hline Sel no. & $\mathrm{n}$ & SPT & WINQ $\div \mathrm{PT}$ & LWKR & BPNN & Optimal & (Eq. No. 5.1) \\
\hline ] & 10 & 27446.3 & 27596 & 26831.5 & 26889.2 & 26075.1 & 3.122 \\
\hline 2 & 15 & 37677.47 & 37709.07 & 36280.73 & 36191.93 & 34558.2 & 4.727 \\
\hline 3 & 20 & 45734.46 & 45676.21 & 43935.94 & 43842.51 & 43053.48 & 1.833 \\
\hline 4 & 25 & 55211.6 & 55025.8 & 52772.55 & 52316.51 & 51516.56 & 1.553 \\
\hline 5 & 30 & 64106.99 & 63345.39 & 60897.44 & 60255.63 & 59386.82 & 1.463 \\
\hline 6 & 35 & 74322.56 & 72803.34 & 70214.73 & 69623.42 & 68469.23 & 1.686 \\
\hline 7 & 40 & 80264.66 & 78441.74 & 75373.9 & 74975.38 & 73573.33 & 1.906 \\
\hline 8 & 45 & 93754.33 & 90887.78 & 86977.52 & 86073.49 & 84897.65 & 1.385 \\
\hline 9 & 50 & 101784.1 & 98259.69 & 94922.15 & 94021.89 & 92624.78 & 1.508 \\
\hline 10 & 55 & 113800.9 & 109890.98 & 106158.4 & 104845.4 & 103785.08 & 1.022 \\
\hline 11 & 60 & 120913.9 & 117916.74 & 113117.8 & 111846.1 & 110364.75 & 1.342 \\
\hline 12 & 75 & 149977.6 & 146410.08 & 139655.6 & 137759.3 & 336286.26 & 1.081 \\
\hline 13 & 85 & 170545.7 & 162467.98 & 156726.2 & 154907.9 & 152938.76 & 1.288 \\
\hline $\mathrm{j} 4$ & 100 & 193658.7 & 188070.2 & 179319.3 & 176437.4 & 174695.08 & 0.997 \\
\hline
\end{tabular}

Table 5.3 Summary of total mean flowtime results of test sets consisting of 50 problems each.

Once again, the neural network results are better as compared to using either SPT, WINQ+PT or LWKR common on all five machines. The network's results deviate from the optimal by an average of $1.74 \%$ (having a minimum of 1.0 to a maximum of $4.7 \%$ ). SPT, PT+WINQ and LWKR results deviate on average by $9.9 \%, 6.25 \%$ and $2.52 \%$ respectively from optimal results.

Similarly, results for all the 50 problems in test set no. $9(n=50)$ are presented in Table 5.4. Also, the comparison between BPNN and optimal mean flowtime is shown graphically in Figure 5.2. It can be observed that BPNN achieves optimal results in $20 \%$ of the problems, but deviates by an average of $2.3 \%$ from optimum in the remainder. Similar comparisons between optimal flowtimes and BPNN results for individual problems in the other thirteen sets are attached in the Appendix E2. 


\begin{tabular}{|c|c|c|c|c|c|c|c|c|c|c|c|}
\hline \multirow{2}{*}{$\begin{array}{c}\text { Datá } \\
\mathrm{sel} \\
\text { No. } \\
\end{array}$} & \multicolumn{4}{|c|}{ Dispatching rule (time units) } & \multirow[b]{2}{*}{ Optimal } & \multirow{2}{*}{$\begin{array}{c}\text { Dala } \\
\text { sel } \\
\text { No. }\end{array}$} & \multicolumn{4}{|c|}{ Dispatching rule (time units) } & \multirow[b]{2}{*}{ Optimal } \\
\hline & $S P^{\prime}$ & WI: & $K R$ & BPNN & & & SPT & WINQ+PT & LWKR & BPNN & \\
\hline $50 \mathrm{FI}$ & 1258.62 & 1126.34 & 1311.6 & 1111.6 & 1092.82 & $50 \mathrm{~F} 26$ & 1774.12 & 1679.06 & 1540.98 & 1540.98 & 1540.98 \\
\hline 5052 & 1822.46 & 178.3 .34 & 1756.04 & 62 & .32 & $50 \mathrm{~F} 27$ & 2363.84 & 2250.56 & 2129.72 & 2129.72 & 5.04 \\
\hline $50 \mathrm{~F} 3$ & 1442.54 & 1378.4 & 1347.78 & & 62 & $50 F 28$ & 1851.14 & 1728.36 & 1666.48 & 1715.28 & 662.92 \\
\hline $50 \mathrm{~F} 4$ & 2051.6 & 1902 & 1826.3 & 1826.3 & 28 & $50 \mathrm{~F} 29$ & 1947.46 & 197 & 1876.34 & 1814.5 & 804.70 \\
\hline $50 \mathrm{~F} 5$ & 2032.9 & 2031 & 1962.68 & 1985.78 & 1930.58 & $50 F 30$ & 2052.44 & 2139.38 & 2073.84 & 2006.34 & 0.0 \\
\hline 5056 & 22015 & $201,20.14$ & 2011.22 & 1928.54 & 1920.02 & $50 \mathrm{~F} 31$ & 1917.3 & .5 & 1810.08 & 1775.84 & 1775.84 \\
\hline $501=7$ & 1779.68 & $182=26$ & 1749.02 & 1673.34 & 1673.34 & $50 \Gamma 32$ & 2280.92 & 207 & 208 & 22 & 70.38 \\
\hline $50 \mathrm{~F} 8$ & 2192.38 & 9.8 & 1941.28 & 1941.28 & 1914.10 & $50 \mathrm{~F} 33$ & 185 & 190 & 68 & 18 & 0.70 \\
\hline $50 \mathrm{F9}$ & 2491.36 & 23131.16 & 2388.74 & 2388.74 & 2276.36 & $50 \mathrm{~F} 34$ & 2115.08 & 2027.34 & 1994.06 & 1994.06 & 2.26 \\
\hline $50 \mathrm{~F} 10$ & 2022.3 & 198 & 1960.96 & .02 & 1877.92 & $50 F 35$ & 2281.58 & 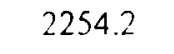 & 205 & 12 & 2.00 \\
\hline $50 F 11$ & 2111.84 & 20514.24 & 197 & 08 & .14 & $50 \mathrm{~F} 36$ & .68 & 18 & 32 & 32 & 3.20 \\
\hline $50 \mathrm{~F} 12$ & 1701.34 & 177 & 1637.22 & 163 & 1579.18 & $50 F 37$ & 2082.58 & 2091.44 & .54 & 48 & .48 \\
\hline $50 \mathrm{~F} 13$ & 3801.34 & 1656.96 & 1638.82 & 3638.82 & 1630.08 & $50 F 38$ & 1961.8 & 1853.54 & 1772.26 & .26 & 1720.42 \\
\hline $50 \mathrm{~F} 14$ & 2254.12 & 221 & 2160.08 & 20 & .16 & $50 \mathrm{~F} 39$ & 2150.1 & 21 & 04 & 56 & .08 \\
\hline $50 \mathrm{~F} 15$ & 2042.28 & 20 & 194 & 19 & 18 & $50 \mathrm{~F} 40$ & 2329.06 & 21 & 2089.64 & .64 & 2080.86 \\
\hline $50 F 16$ & 2327.5 & 2147.8 & 2092.14 & 2134.22 & 2062.82 & $50 \mathrm{~F} 41$ & .58 & 1939.46 & 1863.86 & 86 & 6.58 \\
\hline $50 \mathrm{~F} 17$ & 2073.26 & 1922.46 & 1878.52 & & 1871.16 & $50 \mathrm{~F} 42$ & $2] 62.28$ & 2011.5 & 1990.16 & 1966.62 & 1966.62 \\
\hline $50 \mathrm{~F} 18$ & 2326.98 & 2236.56 & 2066.92 & 2067.68 & 2066.92 & $50 \mathrm{~F} 43$ & 2208.6 & 2020.3 & 1956.88 & .1981 .08 & 1946.96 \\
\hline $50 \mathrm{~F} 19$ & 2121.58 & 19 & 1962.84 & 196 & 1926.58 & $50 \mathrm{~F} 44$ & 2138.88 & 2041.98 & 1982.9 & 2.9 & 1982.90 \\
\hline $50 \mathrm{~F} 20$ & 1847.28 & 1929.04 & ] 695.08 & 1651.9 & 1651.90 & $50 F 45$ & 2351.62 & 16 & 2042 & 2045.08 & 2016.58 \\
\hline $50 F 21$ & 2062.26 & 1979.3 & 1884.82 & 1857.3 & $1857.30 \mid$ & $50 \mathrm{~F} 46$ & 1921.72 & 1867.52 & 1812.36 & 1830.46 & 1798.40 \\
\hline $50 \mathrm{~F} 22$ & 1796.18 & 1902.14 & 1837.14 & 1730.46 & 1729.62 & $50 F 47$ & 1858.82 & & & 1688.36 & 1681.46 \\
\hline $50 F 23$ & 1916.54 & 1930.78 & 1873.16 & 1727.46 & 1727.46 & $50 \mathrm{~F} 48$ & 2229.18 & 2051.36 & 1984.04 & 2011 & 1972.10 \\
\hline $50 F 24$ & 2302 & 2102.06 & 2088.08 & 2088.08 & 2060.26 & $50 F 49$ & 1799.56 & 1677.2 & 1708.88 & 1669.98 & 1642.24 \\
\hline \multirow[t]{2}{*}{$50 \mathrm{~F} 25$} & 2214.38 & 2212.34 & 2125.26 & 2055.04 & 2055.04 & $50 \mathrm{~F} 50$ & 2125.22 & 2136.08 & 2016.88 & 2023.5 & 1959.56 \\
\hline & & & & & & Total & 101784.1 & 98259.69 & 94922. & 94021.89 & 92624 \\
\hline
\end{tabular}

Table 5.4 Mean flowtime for individual test problems for $n=50$ (set no. 9 in Table 5.3).

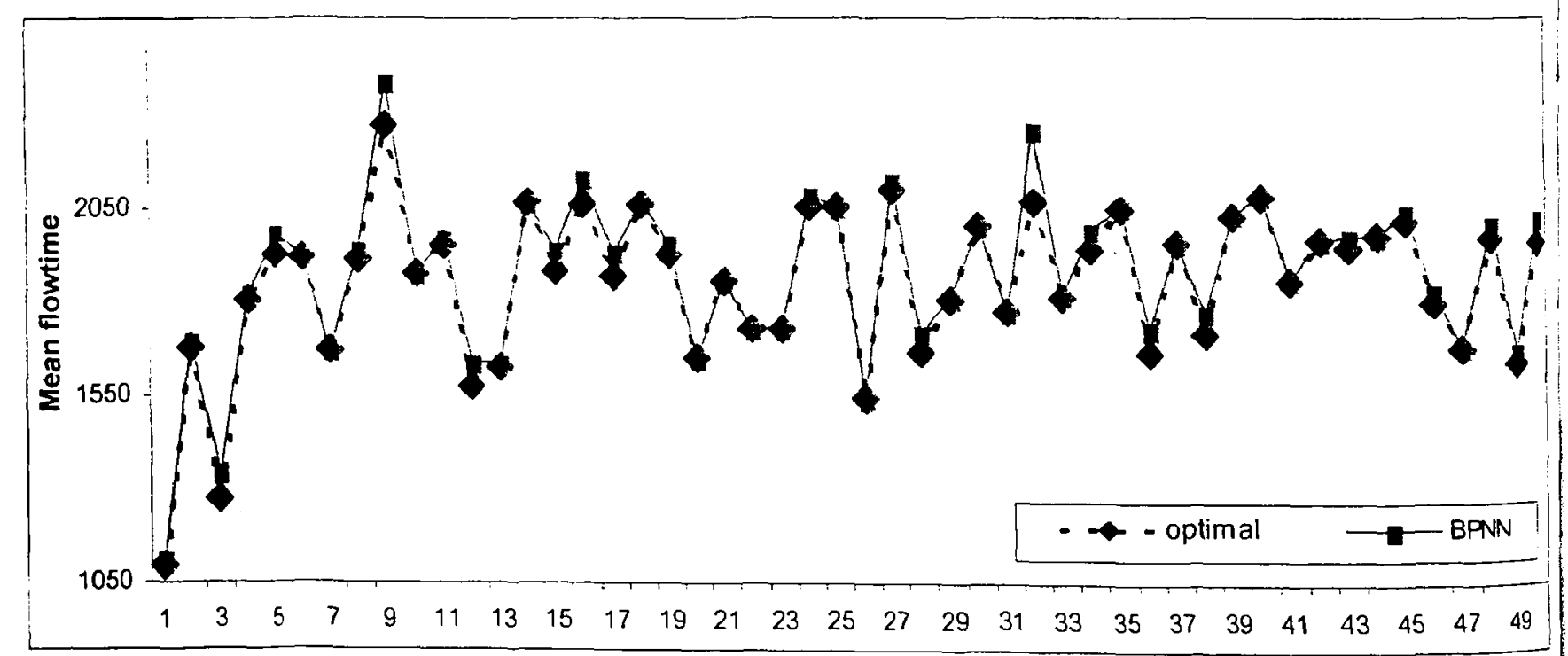

Fig. 5.2 BPNN mean flowtime compared to mean flowtime from optimal rule combinations for $n=50$. 


\subsection{Further Discussion on Results}

Computational results from Table 5.] and Appendix El indicate that the trained neural network has the capability of selecting an appropriate combination of dispatching rules in more than $75 \%$ of all the 700 problem instances for the performance criterion of minimizing makespan. Moreover, the neural network provides the required result more quickly as compared to enumerating all possible combinations. Further, the efficacy of the proposed neural network approach to find the best combination of dispatching rules does not significantly decrease as the number of jobs increase. Figure 5.3 compares BPNN results with the total makespan of the optimal combinations carried out for the various number of jobs ( $\mathrm{n}$ ) (ranging from a minimum of 10 to a maximum 100) for a 5 machine job shop. It can be seen that BPNN performed consistently better than the three other dispatching rules for all $n$.

Figure. 5.3 portrays that, as the number of jobs increases, the difference between BPNN and other dispatching rules decreases (particularly in the case with SPT). This explains SPT's improved performance under higher shop congestion levels; however, this does not imply that the trained network is weakening. In case of SPT, the job with the smallest processing time is given preference over other jobs so that the amount of time and the number of jobs waiting in the queue are reduced, and thus the desired objective of minimizing makespan is achieved well.

Further observations from Figure 5.3 show that, as the number of jobs increases,

SPT also improves in comparison to MWKR. This is because jobs with a larger total remaining processing time may be waiting in queue for a longer time as compared to the jobs with the smallest processing time when SPT is used. Also, the neural network can be 
seen as gradually losing its advantage for the larger number of jobs due to the improved performance of SPT for larger $n$.

For the performance objective of minimizing the mean flowtime, a study is carried out using the results from Table 5.3. The difference between optimal and BPNN mean flowtime for the number of jobs (n) ranging from a minimum of 10 to a maximum of 100 is presented in Figure 5.4 (on the next page).

It is evident from Figure 5.4 that the neural network's effectiveness is still maintained for large size problems. It is also observed that LWKR works well for both small and large size problems. However, the trained neural network proves that a combination of three competing dispatching rules in the job shop generate better mean flowtime for both small and large size problems. It is also evident from this study that the neural network is fairly consistent while others tend to improve only when n gets bigger.

From Figures 5.3 and 5.4 there is some indication that MWKR (for makespan minimization) and LWKR (for flowtime minimization) become more competitive with respect to the BPNN as n grows large. Therefore, it is of interest to determine whether the difference between these dispatching rules and BPNN is significantly different in job shops processing a large number of jobs. This is done by means of ANOVA for the results from the largest problems tested, the 100-job problems.

\subsection{Analysis Of Variance (ANOVA)}

The trained neural network is tested with different sets of jobs. It is desirable to test the significance in the difference between the observed results. The technique used on the post-trained neural network is a one factor ANOVA where a comparison of all means is 


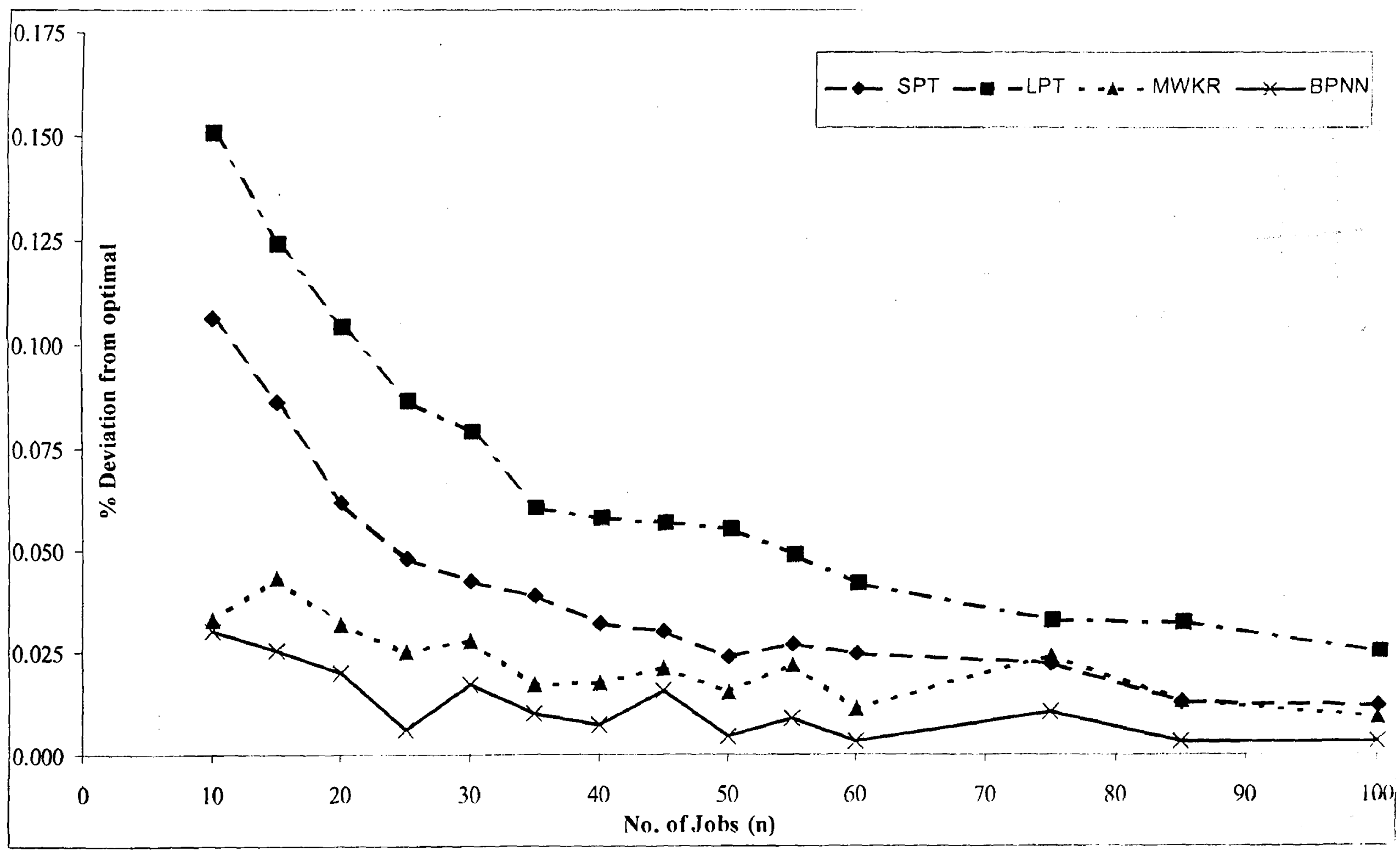

Fig. 5.3 Comparison of BPNN, SPT, LPT and MWKR with respect to optimal total makespan (ref. Table 5.1). 


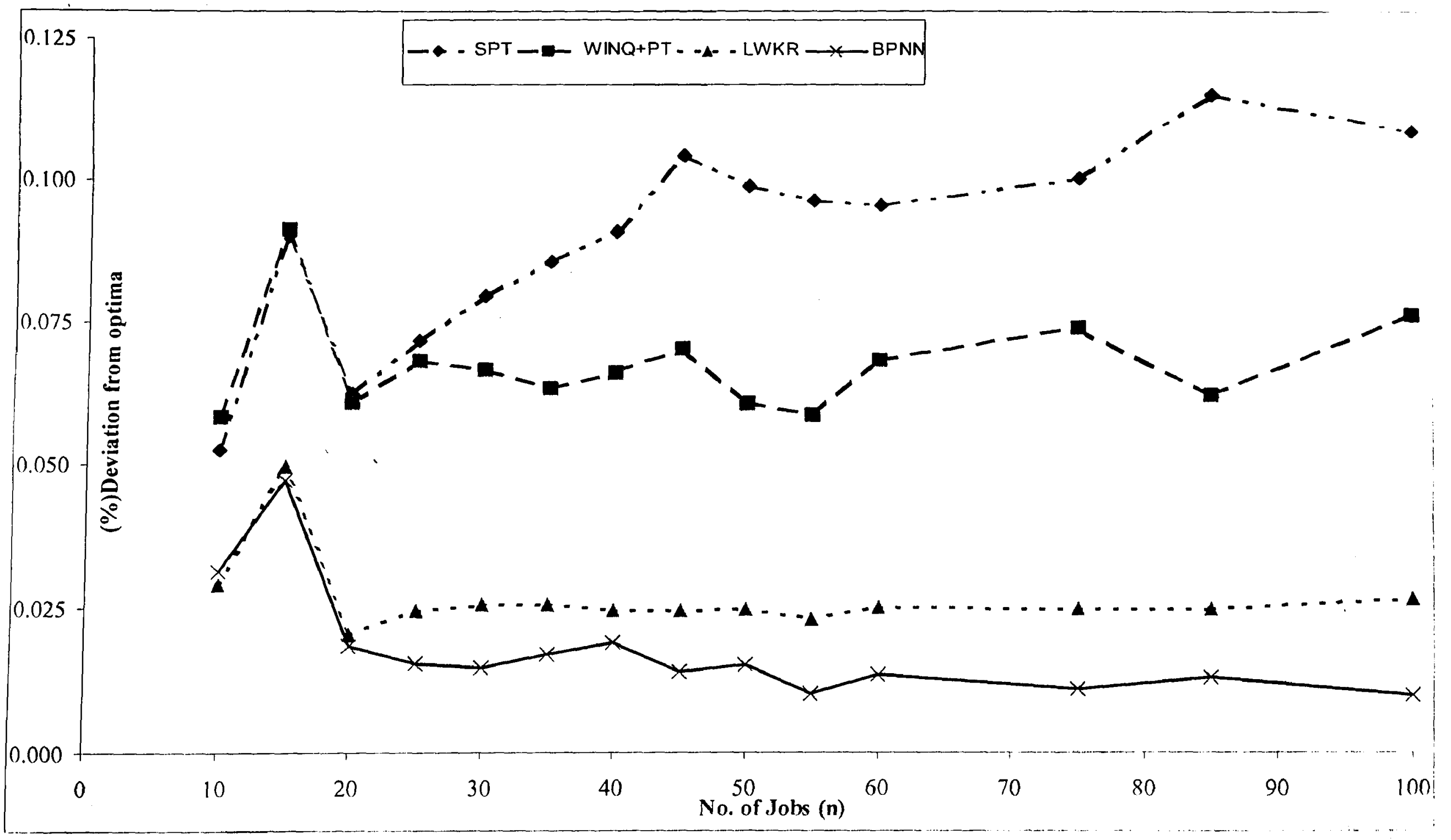

Fig. 5.4 Comparison of BPNN, SPT, WINQ+PT and LWKR with respect to optimal total mean flowtime (ref. Table 5.3). 
done separately for the two objectives of minimizing makespan and mean flowtime. This means to formulate a hypothesis test and to decide whether or not there is sufficient evidence to reject a null hypothesis that there is no significant difference between the means.

While designing the experiment, the objective was to compare the performances of dispatching rules, which includes either single or a combination of dispatching rules. The " between dispatching rules" source of variation, which may be assumed to be due to changes in dispatching rules or a combination of dispatching rules, is of a different kind from that due to sampling and analytical errors. Following are the assumptions considered while carrying out the one factor ANOVA.

\subsubsection{Assumptions}

- The population from which the samples obtained are normally distributed.

- The populations have the same standard deviation $(\sigma)$.

\subsubsection{Data Representation}

In this case, there are four different treatments $(K=4)$, the first three representing different dispatching rules and the fourth one representing a neural combination of dispatching rules. Consider 10 different random samples. Each sample is composed of 50 problems for $n=100$ (as explained previously in section 5.6). Each entry is categorized as a value of $x_{i j}$, which is the average makespan or flowtime on the $j^{t h}$ individual problem taken from $i^{\text {th }}$ sample. 


\subsubsection{General Logic - Analysis Of Variance}

In analysis of variance, between-group variance (difference between treatments) is tested for dissimilarity from within group variance. If the null hypothesis is true, both the above-mentioned variances are about the same, and all the variation can be attributed to random variation. On the other hand, if the between-group variance in comparison to the within-group is larger, then chances are that the samples do not come from populations with equal means. Together, the within-group and between-group variations are the two sources that contribute to the total variation. $F$ is a statistic that represents ratio of two variances:

$F_{\text {ratio }}=\frac{\text { Between }- \text { group Variance }}{\text { Within }- \text { group Variance }}$

If the group means are equal, then $F$ will equal 1.0 and the null hypothesis can be accepted. However, when the sample means are different, the difference can be attributed to the effect of the independent variable plus the sampling error. Thus,

$F_{\text {ratio }}=\frac{\text { Effect of independent variable }+ \text { Sampling error }}{\text { Sampling error }}$

\subsubsection{ANOVA Calculations}

A one-way ANOVA studies the effect of a single independent variable on a dependent variable. The computational formula for the $F_{\text {statistic }}$ involves the sum of squares, which is the sum of squared deviations around the mean. There are two sum of squares that are considered: (1) the total of the sum of squares within the groups $\left(\mathrm{SS}_{10 \mathrm{ta}}\right)$; this is based on the deviation between each observation and $\overline{X_{. .}}$, and (2) the sum of squares for the group mean relative to the grand mean ( $\left.\mathrm{SS}_{\text {group }}\right)$. Thus, 
(a) $\mathrm{SS}_{\text {tolat }}=\sum x^{2}-\frac{\left(\sum x\right)^{2}}{N}$

(b) $\left(\mathrm{SS}_{\text {group }}\right)=\sum n_{j}\left(\overline{X_{j}}-\bar{X}\right)^{2}$

Where, $\bar{X}$ is the grand mean.

$\overline{X_{j}}$ is the group mean.

(c) The total variance is composed of $\mathrm{SS}_{\text {group }} \& \mathrm{SS}_{\text {error. }}$ so that

$\mathrm{SS}_{\mathrm{error}}=\mathrm{SS}_{1 \text { otal }}-\mathrm{SS}_{\text {group }}$

Next is the computation of mean square obtained by dividing the sum of the squared deviations by the degrees of freedom. Thus,

(d) $M S_{\text {group }}=\frac{\text { SSgroup }}{d f_{\text {group }}}$

(e) $\mathrm{MS}_{\text {error }}=\frac{\mathrm{SS} \text { error }}{\mathrm{df} \text { error }}$

The degrees of freedom are:

(f) $d f_{\text {group }}=\mathrm{K}-1$ degrees of freedom between groups.

(g) $\mathrm{df}_{\text {tolal }}=\mathrm{N}-1$ degrees of freedom within groups.

(h) $\mathrm{df}_{\text {eтог }}=\mathrm{N}-\mathrm{K}$

The F. statistic is the ratio of the group mean square to the mean square error.

Thus,

$$
F .=\frac{\mathrm{MS}_{\text {group }}}{\mathrm{MSerror}}
$$




\subsubsection{Hypothesis}

For the one factor ANOVA the null hypothesis is that the population means $(\mu)$ are equal, which is stated as follows:

$$
\mathbf{H}_{0}: \mu_{1}=\mu_{2}=\ldots=\mu_{t}
$$

And the alternative hypothesis is:

$\mathbf{H}_{1}$ : At least two means are significantly different.

There are several alternative ways in which the null hypothesis may be false. For example, only $\mu_{1} \neq \mu_{2}$ or $\mu_{3} \neq \mu_{4}$ or all four population means are unequal, and so on. The alternative hypothesis does not distinguish among these various possibilities, but rather asserts that a relationship exists between the independent and dependent variables such that the population means are not equal.

\subsection{ANOVA Results For Makespan}

The experiment is carried out with 10 samples, each sample consisting of fifty 100-job problems. Thus, 500 problems are considered for this experiment. Table 5.5 presents the average makespan in each of the samples, where three independent dispatching rules are applied exclusively, along with the combination of dispatching rules suggested by the neural network (BPNN).

The ANOVA is performed using the single factor ANOVA function in Microsoft Excel with a level of significance $\alpha=0.05$. Table 5.6 displays the ANOVA results.

From the above results, the $F_{o}$ statistic, $F>F_{0.05,3,36}$, and therefore the nul] hypotheses $\mathrm{H}_{\mathrm{O}}$ can be rejected. It may be concluded that a significant difference exists among the dispatching rules for the performance objective of minimizing makespan. 


\begin{tabular}{|c|c|c|c|c|}
\hline Sample No. & SPT & LPT & MW/KR & BPNN \\
\hline 1 & 7133.1 & 7247.74 & 7142.52 & 7082.22 \\
2 & 7236.66 & 7336.36 & 7288.96 & 7218.88 \\
3 & 7190.48 & 7310.94 & 7230.04 & 7144.34 \\
4 & 7247.64 & 7377.3 & 7246.56 & 7202.06 \\
5 & 7100.54 & 7246.58 & 7238.24 & 7116.42 \\
6 & 7090.24 & 7234.74 & 7202.48 & 7082.54 \\
7 & 7257.36 & 7367.54 & 7231.96 & 7185.02 \\
8 & 7157.24 & 7237.14 & 7197.3 & 7116.6 \\
9 & 7228.22 & 7369.08 & 7232.66 & 7165.82 \\
10 & 7188.88 & 7312.96 & 7231.38 & 7172.62 \\
\hline
\end{tabular}

Table 5.5 Experimental data for ANOVA (average makespan).

SUMMARY

\begin{tabular}{|c|c|c|c|c|}
\hline Groups & Count & Sum & Average & Variance \\
\hline SPT & 10 & 71830.36 & 7183.036 & 3697.794649 \\
LPT & 10 & 73040.38 & 7304.038 & 3394.519951 \\
MWKR & 10 & 72242.1 & 7224.21 & 1441.709533 \\
BPNN & 10 & 71486.52 & 7148.652 & 2316.117351 \\
\hline
\end{tabular}

ANOVA

\begin{tabular}{|c|c|c|c|c|c|c|}
\hline $\begin{array}{c}\text { Source of } \\
\text { Variation }\end{array}$ & SS & df & MS & F & P-value & F crit \\
\hline $\begin{array}{c}\text { Between } \\
\text { Groups }\end{array}$ & 134363.4292 & 3 & 44787.80973 & 16.51141962 & $6.5068 \mathrm{E}-07$ & 2.866265447 \\
\cline { 1 - 4 } $\begin{array}{c}\text { Within } \\
\text { Groups }\end{array}$ & 97651.27336 & 36 & 2712.535371 & & & \\
\hline Total & 232014.7026 & 39 & & & & \\
\hline
\end{tabular}

Table 5.6 ANOVA results for the performance objective of minimizing makespan.

However, it is still unknown which rules are significantly different from the others.

Therefore, a multiple comparison procedure using Fisher's Least Significant Difference (LSD) method (also referred to as the protected t-test) is carried out to compare two group means simultaneously. This tests the significant difference between the means. The formula for the LSD procedure is:

$$
t=\frac{\left(\overline{x_{1}}-\overline{x_{2}}\right)}{\sqrt{M S_{\mathrm{w}}}\left(\frac{1}{n_{1}}+\frac{1}{n_{2}}\right)}
$$

Where $\overline{x_{1}}, \overline{x_{2}}=$ mean for group 1 and group 2 respectively.

$\mathrm{MS}_{\mathrm{error}}=$ mean square within groups (from ANOVA result). 
$n_{1} \cdot n_{2}=$ Sample sizes for groupl and 2 .

In this case BPNN results are compared with the results of all three different dispatching rules and the computation of Fisher's LSD is presented in Table 5.7

\begin{tabular}{|c|c|c|c|c|}
\hline & BPNN & SPT & LPT & MWKR \\
\hline Mean & $\overline{y_{1}}=7148.652$ & $\overline{y_{2}}=7183.036$ & $y_{3}=7304.038$ & $y_{4}=7224.21$ \\
\hline Sample size & $n_{1}=10$ & $\mathrm{n}_{2}=10$ & $n_{3}=10$ & $\mathrm{n}_{4}=10$ \\
\hline $\mathrm{MS}_{\text {error }}$ & \multicolumn{4}{|c|}{2712.535371} \\
\hline Pairs & \multicolumn{4}{|c|}{ Calculation of protected $t$} \\
\hline $\begin{array}{l}\text { BPNN and } \\
\text { SPT }\end{array}$ & \multicolumn{4}{|c|}{$t=\frac{(7148.652-7183.036)}{\sqrt{2 * 2712.535}}=-1.476$} \\
\hline $\begin{array}{l}\text { BPNN and } \\
\text { LPT }\end{array}$ & \multicolumn{4}{|c|}{$t=\frac{(7148.652-7304.38)}{\sqrt{2 * 2712.535}}=-6.671$} \\
\hline $\begin{array}{l}\text { BPNN and } \\
\text { MWKR }\end{array}$ & \multicolumn{4}{|c|}{$l=\frac{(7148.652-7224.21)}{\sqrt{\frac{2 * 2712.535}{10}}}=-3.244$} \\
\hline
\end{tabular}

Table 5.7 LSD calculations for the minimization of makespan experiment.

The critical value for 1 at a significance level $\alpha=.05$ and $\mathrm{df}=36$ is $t=2.02$ (from the standard t distribution table) (Montgomery, 1997).

The protected t-tests show significant differences between LPT and MWKR with respect to BPNN. On the other hand, there is no evidence of a significant difference between the group mean of SPT and BPNN. This means that SPT might be improving as the problem size increases. The results could also indicate that the neural network may be getting weaker as n grows. Similarly, it has been observed that there is a significant difference between other groups of means such as MWKR and LPT, except between SPT and MWKR. Thus, it is further evident that SPT improves as the number of jobs increase, as compared to both BPNN and MWKR. 


\subsection{ANOVA Results For Mean Flowtime}

A similar ANOVA analysis is also carried out for the mean flowtime result. The details of the experiment and the ANOVA results are presented in Table 5.8 and Table 5.9 respectively.

\begin{tabular}{|c|c|c|c|c|}
\hline Sample No. & SPT & WINO-PT & LWKR & BPNN \\
\hline 1 & 3827.6104 & 3721.1716 & 3563.1432 & 3512.494 \\
2 & 3902.2484 & 3762.8834 & 3597.3018 & 3545.122 \\
3 & 3900.6868 & 3780.1184 & 3646.3354 & 3583.527 \\
4 & 3944.2476 & 3773.6718 & 3619.7972 & 3578.526 \\
5 & 3931.0576 & 3838.7032 & 3655.1484 & 3605.348 \\
6 & 3963.7254 & 3827.3976 & 3677.554 & 3617.025 \\
7 & 3861.4982 & 3734.0116 & 3567.4578 & 3525.153 \\
8 & 3879.2966 & 3773.5424 & 3594.816 & 3535.001 \\
9 & 3924.7204 & 3783.5388 & 3634.73 & 3577.858 \\
10 & 3913.9456 & 3767.9396 & 3624.6554 & 3565.213 \\
\hline
\end{tabular}

Table 5.8 ANOVA experimental data (average mean nowtime).

SUMMARY

\begin{tabular}{|c|c|c|c|c|}
\hline Groups & Count & Sum & Average & Variance \\
\hline SPT & 10 & 39049.037 & 3904.9037 & 1633.918554 \\
WINQ+PT & 10 & 37762.9784 & 3776.29784 & 1295.996328 \\
LWKR & 10 & 36180.9392 & 3618.09392 & 1398.511358 \\
BPNN & 10 & 35645.2654 & 3564.52654 & 1184.850026 \\
\hline
\end{tabular}

ANOVA

\begin{tabular}{|c|c|c|c|c|c|c|}
\hline $\begin{array}{c}\text { Source of } \\
\text { Variation }\end{array}$ & SS & df & MS & F & P-value & F crit \\
\hline $\begin{array}{c}\text { Between } \\
\text { Groups }\end{array}$ & 718502.3905 & 3 & 239500.7968 & 173.7629571 & $1.8166 \mathrm{E}-21$ & 2.866265447 \\
\hline $\begin{array}{c}\text { Within } \\
\text { Groups }\end{array}$ & 49619.4864 & 36 & 1378.319067 & & & \\
\hline Total & 768121.8769 & 39 & & & \\
\hline
\end{tabular}

Table 5.9 ANOVA results for the performance objective of minimizing mean fowtime.

From the ANOVA results, since $F>F_{0.05 .3 .36}$, the null hypothesis can be rejected and it can be concluded that there is a significant difference among the mean of different dispatching rules and BPNN in minimizing the mean flowtime. Fisher's LSD procedure is also carried out and Table 5.10 depicts these calculations.

The protected t-tests show that there is a significant difference between BPNN on 


\begin{tabular}{|c|c|c|c|c|}
\hline & BPNN & SPT & WINQ +PT & LWKR \\
\hline Mean & $y_{1}=3564.53$ & $y_{2}=3904.90$ & $y_{3}=3776.29$ & $\overline{y_{4}}=3618.09$ \\
\hline Sample Size & $n_{1}=10$ & $n_{2}=10$ & $\mathrm{n}_{3}=10$ & $n_{4}=10$ \\
\hline MS error & \multicolumn{4}{|c|}{1378.319} \\
\hline Pairs & \multicolumn{4}{|c|}{ Calculation of protected $t$} \\
\hline $\begin{array}{l}\text { BPNN and } \\
\text { SPT }\end{array}$ & \multicolumn{4}{|c|}{$t=\frac{(3564.53-3904.90)}{\sqrt{2 * 1378.319}}=-20.501$} \\
\hline $\begin{array}{l}\text { BPNN and } \\
\text { WINQ+ PT }\end{array}$ & \multicolumn{4}{|c|}{$t=\frac{(3564.53-3776.29)}{\sqrt{2 * 1378.319}}=-12.755$} \\
\hline $\begin{array}{l}\text { BPNN and } \\
\text { LWKR }\end{array}$ & \multicolumn{4}{|c|}{$t=\frac{(3564.53-3618.09)}{2 * 1378.319}=-3.226$} \\
\hline
\end{tabular}

Table 5.10 LSD calculations for the mean flowtime experiment.

one hand, and all three dispatching rules, SPT, WNQ+PT and LWKR on the other.

In this chapter, the performances of different dispatching rules including BPNN are compared with the optimal results for various problem sizes. The results show that BPNN performs better than individual dispatching rules. The BPNN results were close to optimal for both minimizing makespan and mean flowtime. Also, for larger size problems, ANOVA tests were performed on both the performance objectives separately. This was for examining significant differences between different groups of means. The results show that for the makespan criterion, in the larger size problem, there is a significant difference between means of BPNN and LPT, and BPNN and MWKR, but not between SPT and BPNN. On the other hand, for the mean flowtime criterion. ANOVA shows a significant difference among all the dispatching rules and BPNN, for the large size problem. In the next chapter, a summary of the results, followed by the conclusion and further research, is discussed. 


\section{CHAPTER 6}

\section{Conclusion and Further research}

\subsection{Summary}

This research has considered the problem of selecting an appropriate combination of dispatching/priority rules for scheduling job shops. The dispatching rule selection problem is shown to be a prediction problem that can be successfully solved using a proposed neural network. For building the neural network, simulations were carried out to constitute a training set. Once the network was trained, it was shown to be able to select well-suited dispatching rules for new problems. Computational results showed that the proposed neural network correctly predicted makespan in more than $75 \%$ of the problem instances. Further, the effectiveness and generalization capability of the proposed neural network was retained with increase in the number of jobs. Results indicated that, for the problem situation considered, the concept of using a combination of dispatching rules for different situations (number of jobs) in a neural network yields better results than using a single dispatching rule. The proposed neural network for minimizing the makespan criterion produced an average improvement of about $1.5 \%$ over MWKR (the best competing individual rule) and 6\% over LPT (the poorest performer among the three rules considered), as seen in Table 5.1. On the other hand, for minimizing the mean flowtime objective, the proposed neural network performed better by an average of $1 \%$ over the best competing individual rule (LWKR), and by $6.5 \%$ over SPT (the poorest performing rule considered), according to the results in Table 5.3. 
In this study, the test problems were randomly generated, and a small fraction of the 120 possible jrob routes for five machines were considered in each problem instance. This simulates a realistic scenario where jobs with the same routes belong to either a part family or a group, and have similar sequences of operations but different processing times.

\subsection{Conclusion}

The proposed neural network is an input-output model. In this research the input to the proposed network consists of total processing time, variance of processing time and the mean routing order on each machine. The trained neural network identifies such characteristics and quickly assigns one of three dispatching rules to use on each machine. It has been observed during the study that if only a single dispatching rule like smallest processing time (SPT) is applied on all five machines, than the optimality of the desired performance objective (in this case minimizing makespan) is not always achieved in the shop. On the other hand, if neural selections of dispatching rules are applied to each of the machines, then better results are obtained as compared to using a single dispatching rule.

Although the neural network was trained with small job numbers, the efficacy of the trained neural network for larger size problems appears to be maintained. For example, the BPNN results deviate only $0.429 \%$ from the total of the makespans achieved by the optimal mix of the three dispatching rules for job shops with $n=50$.

Hence, the BPNN, from a practical application point of view, can be used as a tool to aid scheduling decisions in a plant, or can be embedded in a production computer integrated manufacturing system for automated and dynamic selection of appropriate 
priority rules. The developed model offers significant advantages regarding time consumption and simplicity for scheduling new job shop problems.

\subsection{Further Research}

The suggested methodology is not restricted to a static job shop model, but there are a number of fruitful directions that can be identified for further research from this study, some of which are:

1) Dynamic job shop problems.

2) Different performance objectives.

3) Relaxation of assumptions.

4) Number of dispatching rules.

5) Job shops with more machines.

The details of each future direction are as follows:

The present study can be extended to consider a dynamic job shop problem, where the arrival times of jobs are not known (in advance) before scheduling. The jobs may arrive at any time; in such a real-world situation, the job characteristics are fed to the neural network. The trained network can decide in real-time when to change rules on specific machines in response to the changing characteristics of the work-in process so as to better meet the performancé objectives. It is worth investigating how this network trained for a static job shop, will perform when subjected to a dynamic environment.

A similar neura] network approach can be used for other performance objectives where the jobs will have an additional characteristic such as due date, like minimizing mean tardiness, minimizing number of tardy jobs or minimizing the cost. The current study made some assumptions, like no machine breakdowns, material-handling systems 
are available at all limes, etc. The neural network can be trained by relaxing such assumptions in order to capture more realistic real world situations. In a flexible manufacturing system that resembles the job shop model, if one of the material handling systcms breaks down. then in such a situation an intelligent neural network can optimize the job sequence and thus the desired performance objective.

In the present study, a set of three dispatching rules was considered for two different performance objectives, minimizing makespan and mean flowtime. The study can be extended to test the possibilities of getting better solutions if combinations of more than three dispatching rules are used for the same or different performance objectives.

In the present study, a five-machine job shop problem was considered; a similar approach can be extended to a higher number of machines, for example, ten machines for the same or different performance objectives.

Summing up, the objective/purpose of this research was to develop and train an artificial neural network to select the best combination among three dispatching rules for a five-machine job shop problem and two different performance criteria. Experimental results showed that the trained neural network was able to successfully predict good rules to use on each machine, leading to better satisfaction of the performance criteria when compared with the alternative of using one identical rule on all machines. 


\section{References}

Baker K. (2002) Elements of sequencing and scheduling. Hanover N.H., ISBN 0963974610.

Bialey D. and Thompson D. (1990) How to develop neural-network. Al expert, vol.5 (3), pp.38-47.

Blackstone J., Phillips D. and Hogg G. (1982) A state of-the-art survey of dispatching rules for manufacturing job shop operations. International journal of production research, vol.20 (1), pp.27-45.

Blazewicz J., Domschke W. and Pesch E. (1996) The job shop scheduling problem: conventional and new solution techniques. European journal of operations research, vol. 93, pp.1-33.

Conway R., Maxwell W.and W Miller L. (1967) Theory of Scheduling. Addison-Wesley.

French S. (1982) Sequencing and scheduling: An introduction to the mathematics of the job shop. John Wiley \& sons Inc., New York.

Foo S.Y. and Takefuji Y. (1988a) Stochastic neural networks for solving job shop scheduling: Part 1, Problem definition. Proceedings of the 1988 IEEE international conference on neural networks, San Diego, vol.2, pp.275-282. 
Foo S.Y. and Takefuji Y. (1988b) Stochastic neural networks for solving job shop scheduling: Part 2, Architecture and simulations. Proceedings of the 1988 IEEE international conference on neural networks, San Diego, vol.2, pp.283-290.

Garson D. (1099) Neural nerworks an introductory guide for social scientists. SAGE publications, ISBN 0-7619-5731-6.

Klimasauskas, C.C. (1992) Applying neural networks in finance and Investing. R.R Trippi and E. Turban (eds), Chicago: Probus, pp.47-72.

Jain A. and Meeran S. (1998) Job-shop scheduling using neural networks. International journal of production research, vol.36 (5), pp.1249-1272.

Jones A., Rabelo L. and Yih Y. (1996) Job shop scheduling. Encyclopedia of operations research, (http://ww'w.mel.nist.gov/msidlibrarv/doc/iobshop1.pdf).

Kiran, A. S. and Smith M. L. (1984) Simulation Studies in Job Shop Scheduling-I. Computer. \& industrial engineering, vol.8, pp.87-93.

Kaastra 1. and Boyd M. (1996) Designing a neural network for forecasting financial and economic time series. Neurocomputing, vol.10 (3), pp.215-236.

Kelton D., Sadowski R and Sadowski D. (2002) Simulation with Arena. McGraw-Hill series, ISBN 0-07-239270-3. 
Kumar H and Srinivasan G. (1996) A genetic algorithm for job shop scheduling - A case study. Computers in industry, vol.31, pp.155-160.

Montgomery D. (1997) Introduction to statistical quality control - Third edition. John Wiley \& Sons, Inc New York.

Neuroshell 2 software manual. Ward systems group, Inc. http://www.wardsystems.com.

Panwalkar S. and Iskander W. (1977) A survey of scheduling rules. Operations research, $\operatorname{vol.25(1),pp.~45-61.~}$

Pierreval H. (1992) Training a neural network by simulation for dispatching problems. IEEE Proceeding of the third rensselear international conference on computer integrated engineering, pp.332-336.

Pierreval H. (1993) Neural network to select dynamic scheduling heuristics. Journal of decision scjence, vol.2 (2), pp.173-190.

Pinadeo M. (1995) Scheduling theory, algorithms and systems. Prentic Call Englewood Cliffs, NJ.

Rabelo L. (1990) A hybrid artificial neural networks and knowledge-based expert systems approach to flexible manufacturing system scheduling. PhD dissertation. 
Raghu. T.S. and Rajendran. (. (1993). An Efficient Dynamic Dispatching Rule for Scheduling in a job shop. International Journal of Production Research, vol.28, pp.2277-2292.

Rajendran C. and Holthaus O. (1999) A comparative study of dispatching rules in dynamic flowshops and job shops. European journal of operational research, vol.116, pp. $156-170$.

Reinhardt M. (1990) Neural networks An Introduction. Springer-verlag, Berlin Heidelberg.

Sabuncuoglo I. and Gurgun B. (1996) A neural network model for scheduling problems. European journal of operational research, vol.93, pp.288-299.

Sabuncuoglo 1. (1998) Scheduling with neural networks: a review of the literature and new research directions. Production planning \& control, vol.9 (1), pp. 2-12.

Satake T., Morikawa K. and Nakamura N. (1994) Neural network approach for minimizing the makespan of the general job-shop. International journal of production economics, vol.33, pp. 67-74.

Sim S.K., Yeo T.Y and Lee W.H. (1994) An expert neural network system for dynamic job shop scheduling. International journal of production research, vol.32 (8), pp.17591773. 
Smith K. (1999) Introduction to Neural networks and data mining for business applications. Eruditions publishing. ISBN 1864910046.

Subramaniam V., Ramesh T., Lee K.G., Wong S.Y. and Hong S G. (2000) Job shop scheduling with dynamic fuzzy selection of dispatching rules. Advanced manufacturing technology, vol.16, pp.759-764.

Swingler K. (1999) Applying Neural networks A project guide. Morgan kaufman publishers, Inc, California.

Waikar A., Saker B. and Lal A. (1995) A comparative study of some priority dispatching rules under different shop loads. Production planning and control, vol.6 (3), pp.301-310.

Zhang H.C and Huang S.H. (1995) Applications of neural networks in manufacturing: a state of art survey. International journal of production research, vol.33 (3), pp.705-728. 


\section{Appendix A I: Problem generation}

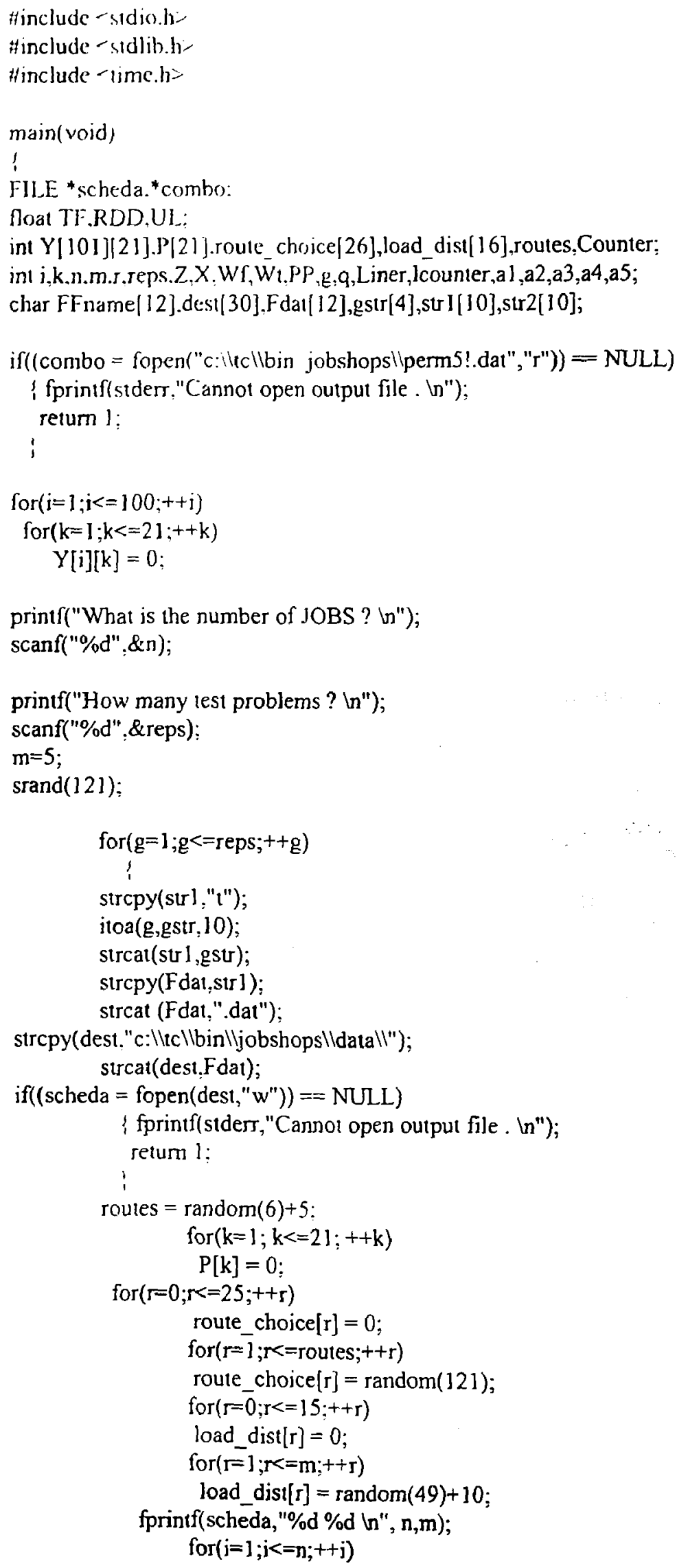




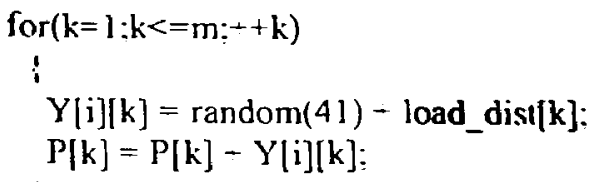

for $(j=1 ; j<=n:++i)$

!

$\mathrm{Z}=1000$

lcounter $=0$;

Liner $=$ route_choice $[$ random $($ routes $)+1]$;

while( Liner $!=$ lcounter)

I fscanf(combo,"\%d \%d \%d \%d \%d In",\&a 1,\&a2,\&a3,\&a4,\&a5);

++ lcounter; ;

fprintf(scheda,"\%d \%d \%d \%d \%d ",al.a2,a3,a4,a5);

rewind(combo);

fprintf(scheda." \%d ",Y[i] [al ]);

fprintf(scheda, "\%d ",Y[i]][a2]);

fprintf(scheda,"\%d ",Y[i][a3]);

fprintf(scheda,"\%d ",Y[i][a4]);

fprintf(scheda,"\%d ",Y[i][a5]);

fprintf(scheda,"lt \%d",Z);

fprintf(scheda," $n ")$;

;

fprintf(scheda," $\ln \backslash n$ ");

\section{fclose(scheda);}

++Counter;

printf("counter iss : \%dln",Counter);

fclose(combo);

\} 


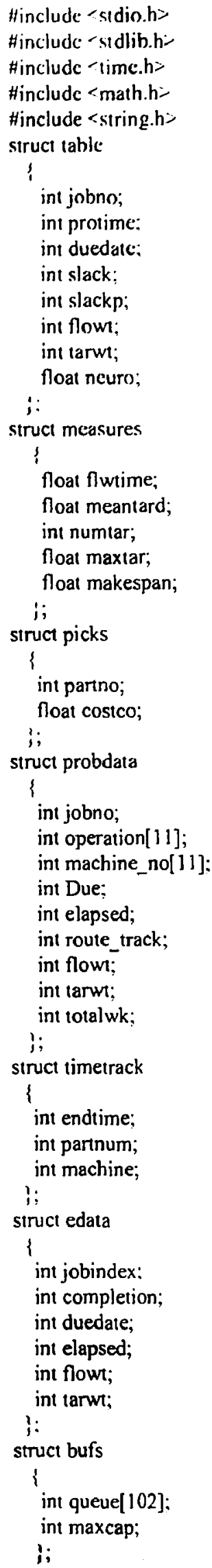




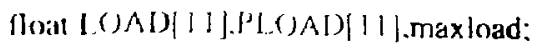

struct mecasures MM[10]:

struct bufs bufferl 11$]$;

struct limetrack event[211]:

struct colata sink $[111]$;

struct probdata PRT|102]:int dispatching_rulef 16];

int objsel,consel:

int TIMENOW.dd.bst;

flnat Tardiness.TTard,Bcost.CCost:

int Count=(), machines, h.v:

char ncal[3]:

int droutput 31$]$ :

main(void)

i

FILE * combo. *sinput. *soutput. * neuinpur. " neuout:

struct tablespt_son().cdd_son().mslk_son().lpt_son().mslp_son(),w.spt_sont();

struct lablc clear_job(),update_jobs().random_sort():

struct measures calculate_measures(),calculate_cost(),schedule_costs():

struct edata find_sched():

struct bufs

spt_buf(),jpt_buf().tw'spt_buf(),CD().tspt_buf(),edd_buf().slack_buf().coven_buf(),mdd_buf(),lwkr_buf(),twk_buf(); struct bufs snq_buf(),winq_buf().append_to_buffer(), unload_machine();

int combox[244][6],kl,k2.k3.k4.k5,ji,combination;

float mc_square[1]], maxsidev;

float best_result,performance;

int remove_job(), max_prot().max_date.member_of(), Avertot;

float Mflow, Bflow;

int $i, k, k i, u, n, p, q, r, t, y, j, j o b$, sequence,next_mac,curmac,best_tardy;

int place, found;

int best_tardiness, best_flow,best_combo.mac_candidate[14],mincostjob,torpro,mincostdue,mondue;

int BEST[300][1]], njobs[3];

int greater_of(),counter,ije_counter;

char FFname[ 12],WWR[44].des12[30].dest ] [30].dest[30].Fdat[12];

char ${ }^{*}$ strl;

int combocounter $=0$;

if $(($ soutput $=$ fopen $(" c:$ iltclibiniljobshopsildata|lresult.dat".,"w")) ==NULL)

\{ fprintf(stderr, "Cannot open ourput file . in");

return 1;

j

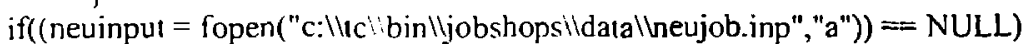

\{ fprintf(stderr,"Cannol open ourput file . $\mathrm{V}^{\text {"I }}$;

relum !;

;

fprintf(soutput,"In M/C I M/C 2 M/C $3 \quad$ M/C 4 M/C 5 (n"):

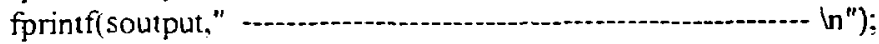

$I^{*}$

printf("Select an Objective Function : $\ln \backslash n ")$ :

printf("1. Minimizing Mean Flowtime $(n ")$ :

printf("2. Minimizing Makespan in"):

scanf("\%d",\&objsel); */

objsel $=2$;

if(objsel==1)

\{

if ((neuout = fopen("c: Iltcllbinlljobshopslidatalneujobf,out","a")) == NULL)

$\{$ fprintf(stderr, "Cannot open output file . $ا$ ");

return 1 ;

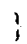

\}

else

\{

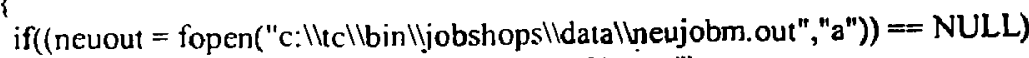

$\{$ fprintf(stderr, "Cannot open output file. $v "$ ");

return l; 


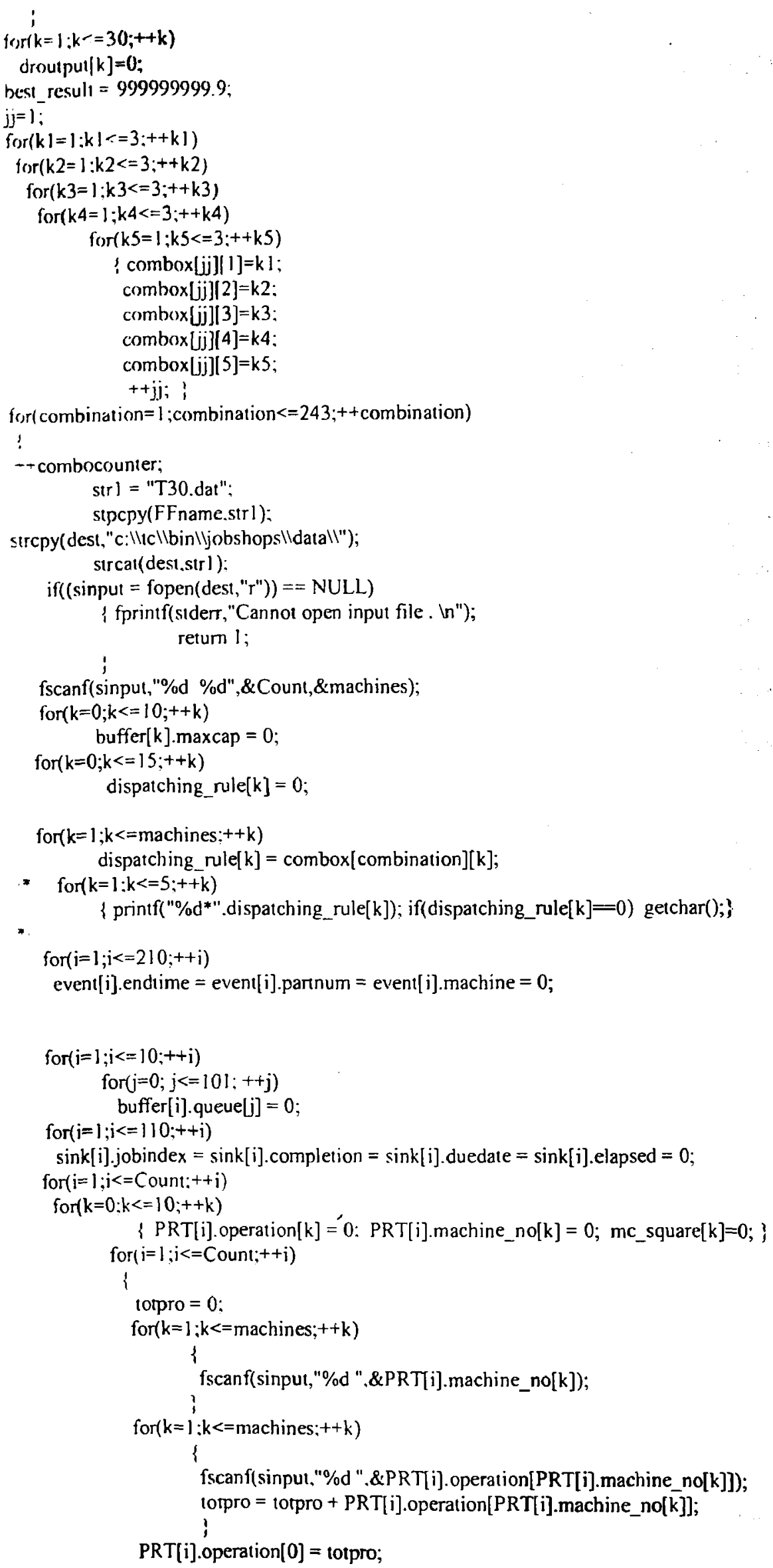




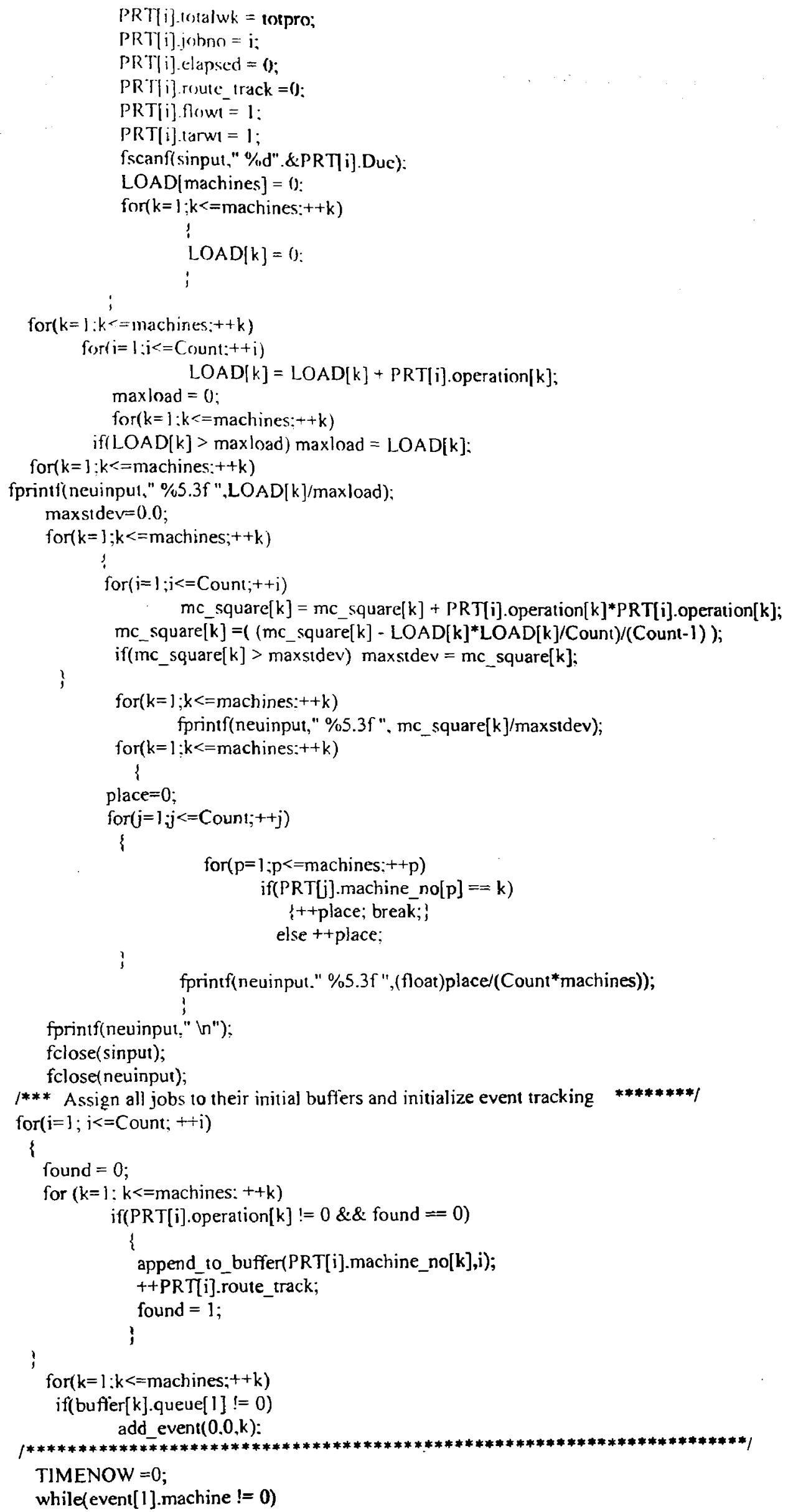


sequence $=\{R T$ event $[1]$.partnum].route_track:

curmac $=$ even1 $[1] \cdot$ machinc:

job=cvent[ ] ].partnum:

1* printf("TIME : \%d - EVENT on machine \%d for PART \%d

in".event[1].enditime.cvent[1].machine.event[1].partnum);

printf("TIMENOW = \%d $\mathrm{k}$ ",event[ ] ].endime); */

TIMENOW = cvent $[1]$.endtime:

$\mathrm{j}=\mathrm{l}$;

while(cvent[i].machine $!=0$ )

?

cvent[i].cndime $=$ event $[j] \cdot$ endime - TIMENOW:

$++\mathrm{j}$;

;

$\operatorname{for}(j=1 ; j<=C$ ount $++j)$

!

PRTLj].elapsed = PRT[i].elapsed + TIMENOW;

PRT[i].Due $=$ PRT[j].Due - TIMENOW:

TIMENOW $=0$;

update_event_list():

iffob $!=0$ )

1

$l^{*}$ move job to the next machine's buffer */

found $=0$;

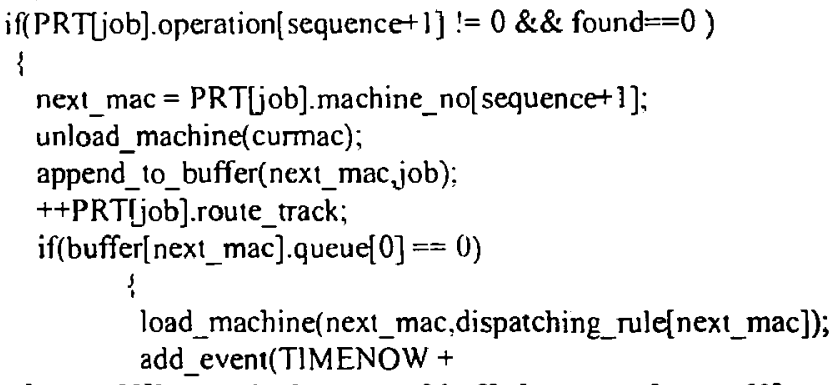

PRT[buffer[nex1_mac].queue[0]].operation[next_mac],buffer[next_mac].queue[0],next_mac);

found $=1$ :

;

if(found $==0$ )

1

add_to_sink(job,TIMENOW, 0 ); unload_machine(curmac); ;

load_machine(curmac,dispatching_rule[curmac]);

if (buffer[curmac] queue[0] $!=0$ )

add event(TIMENOW +

PRT[buffer[curmac].queue[0]].operation[cumnac],buffer[curmac].queue[0],curmac);

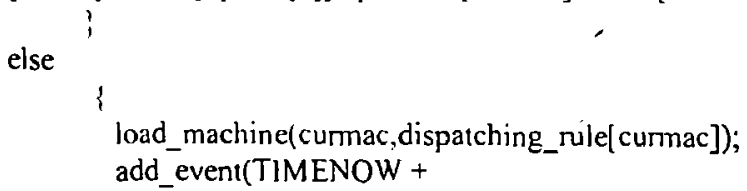

PRT[buffer[curmac].queue[0]].operation[curmac],buffer[curmac].queue[0],curmac);

I $l^{*}$ end of combination for loop schedule costs():

1* printf("mean flowtime $=\% 7.2 \mathrm{f} \mathrm{n}$ ".MM[1].flwtime): printf("makespan =\%7.2f $\mathrm{n} " . \mathrm{MM}[1]$.makespan);

printf("number of jobs tardy = \%d In".MM[1].numtar);

printf("maximum job tardiness = \% 7.2f $\ln$ ",MM[1].maxtar);

printf("In");

for $(j=1 ; j<=$ machines: $++i)$ 
printf("The maximum qucue length in buffer $\% d=\% d$ unints $\mathrm{ln} ", \mathrm{i}$, bufferli].maxcap); printf(" n"):

*1

if(objisel $= \pm 1$ ) performance $=$ MM[1].thutime:

iff performance - best_result -0.00001 )

: best_result = performance:

ije counter $=1$;

for $(u=1 ; u<=$ machines; $++u)$

BEST/tic_counter] [u] = dispatching_rulefu]; ;

clse if (performance $>$ best_result-0.00001 \&\& performance $<$ best_result +0.00001 )

?

++ ije counter:

for $(\mathrm{u}=1: \mathrm{u}<=$ machincs: $++\mathrm{u})$

BEST[tie_counter] $\mid u]=$ dispatching_rulefu]; :

: $/ * * * *$ end of the main white $* * * * \mid$

for $(p=1 ; p<=$ ie_counter; $++p)$

!

for $(u=1: u<=$ machines: $++u)$

$!$

if(BEST[p][u] == 1) ; fprintf(soutput," SPT "); ++ droutput [3*u-2]; ;

if(BEST[p][u] == 2) \{ fprintf(soutput," LPT "); ++ droutput[ $\left[3^{*} u-1\right] ;$;

if(BEST[p][u] == 3) \{fprintf(soutput," MWKR "); ++droutput[3*u]; \} ;

fprintf( soutput," "n ");

;

fprintf(soutput,"

if (objsel $==1$ )

fprintf(soutput," mean flowime $=\% 7.2 \mathrm{f}$ ",best_result);

e]se

fprintf(soutput," makespan $=\% 7.2 \mathrm{f}$ ".best_result);

for $(i=1 ; i<=3 *$ machines; $++i)$

fprintf(neuout," \%5.2f".(float)droutput[i]/tie_counter);

fprintf(neuout," $\ln "$ ");

fclose(neuout);

fclose(combo);

fclose(soutput):

return $(0)$;

;

struct bufs append_to_buffer(bnum,jobnum)

int bnum, jobnum;

\{

int $\mathrm{j}=1$;

while(buffer[bnum].queue[i] $!=0$ )

$++\mathrm{i}$

::

if ( $\mathrm{i}>$ buffer[bnum] maxcap) buffer[bnum].maxcap $=\mathbf{i}$;

buffer[bnum].queue[i] = jobnum:

retum;

'

* Function: unload_machi( ) This function removes a job from machine (t)

struct bufs unload_machine(finmac)

int finmac;

\{

int i.finjob:

finjob = buffer[finmac].queue[0];

LOAD [finmac] = LOAD[finmac] - PRT[finjob].operation[finmac];

PRT[finjob].operation[0] = PRT[finjob].operation[0] - PRT[finjob].operation[finmac];

buffer[finmac].queue $[0]=0$ :

return;

) 


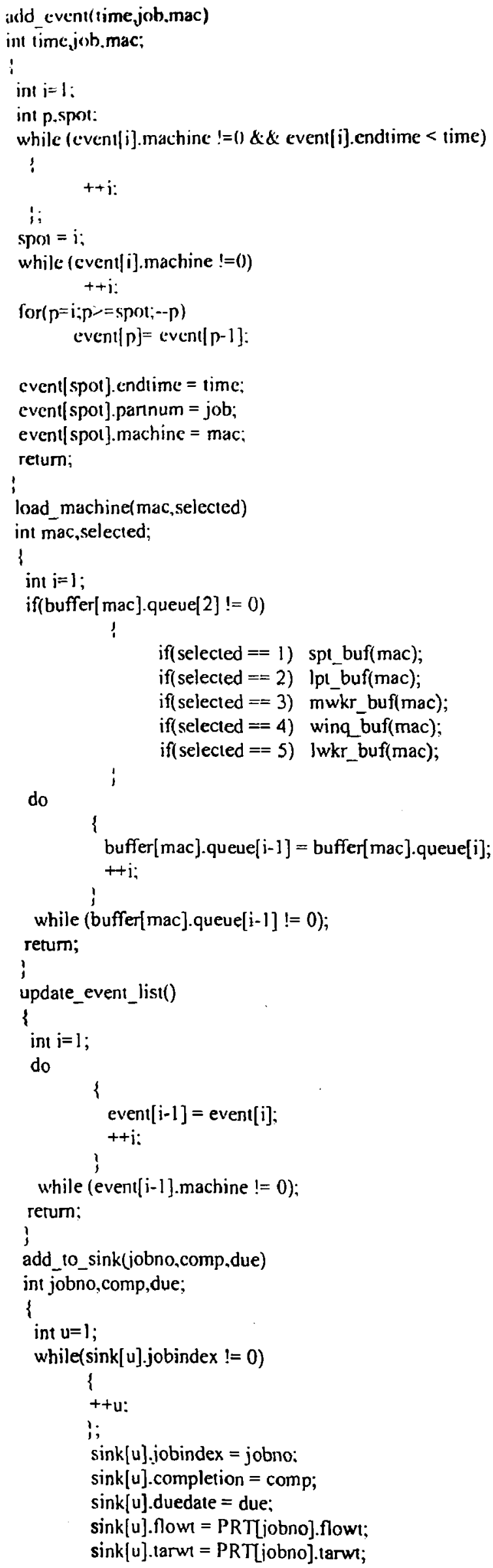




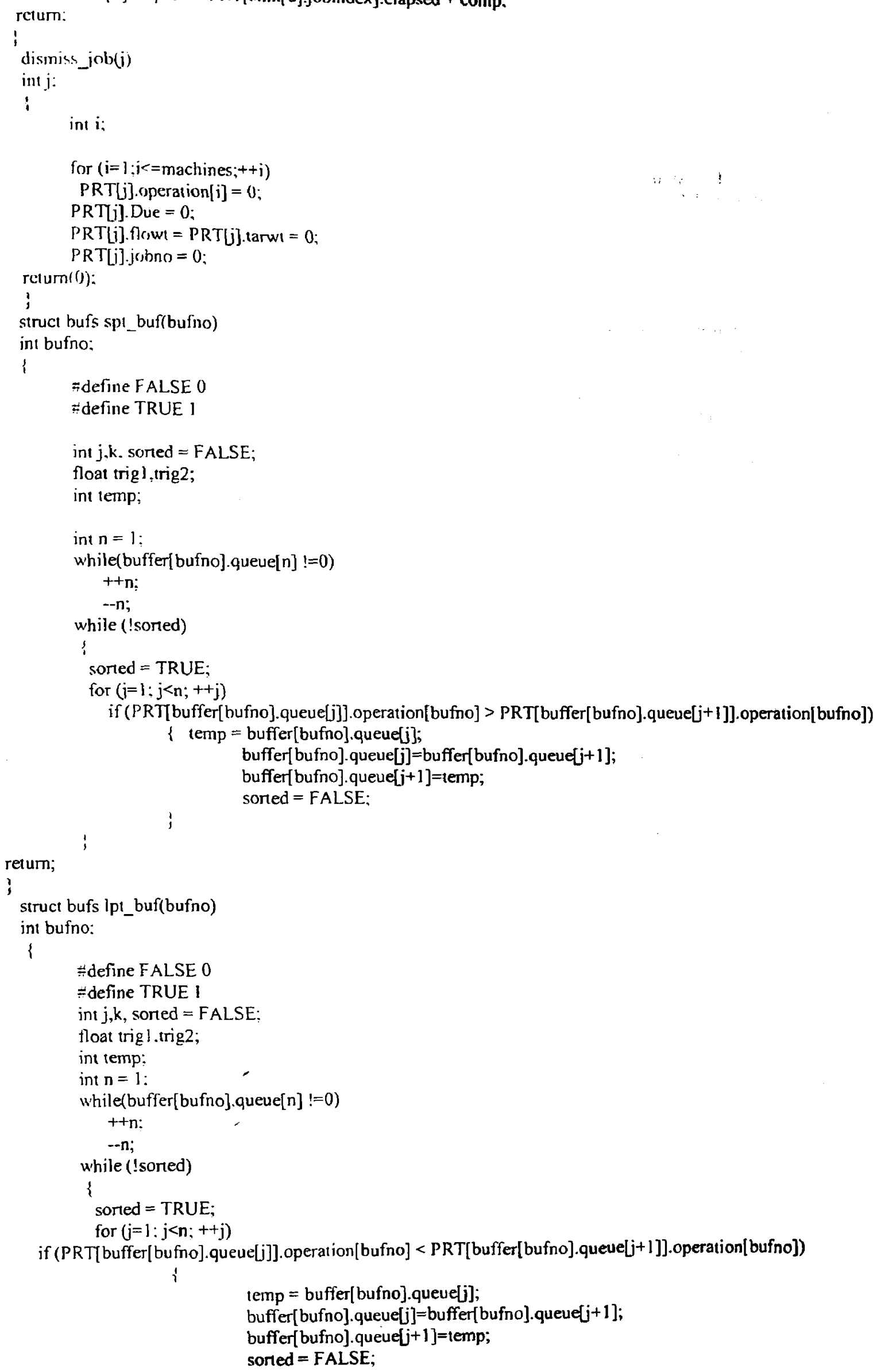




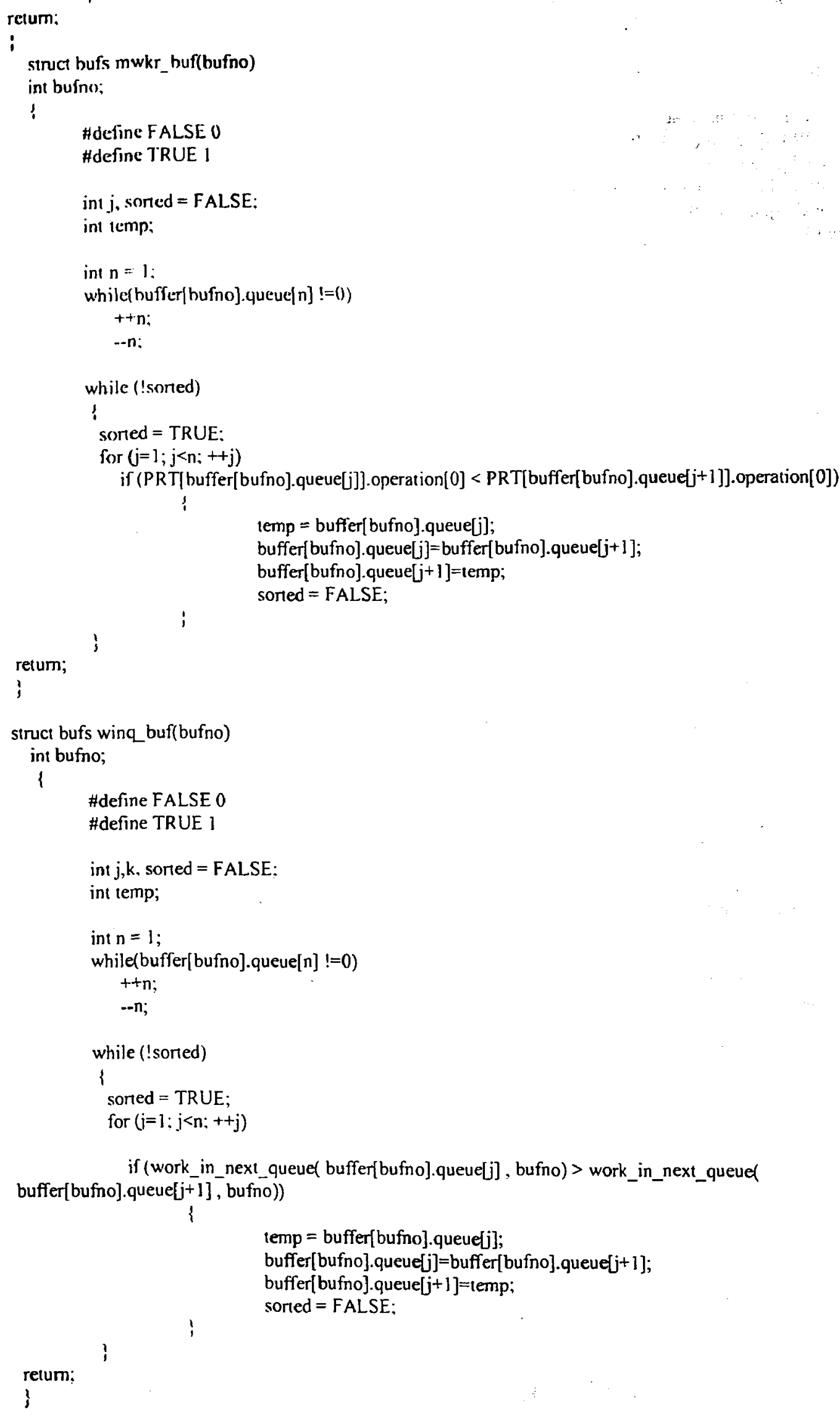


work_in_next_queuc(jobnumber,present_mac)

int jobnumber, present_mac:

1

int $y . k]$ numb.wailing_ish.next_mac, workinqueuc=0;

for $(q=): q<=($ ount: $++q)$

if(PRT[q].johno $==$ jobnumber) break:

for $(k]=1 ; k]<$ machines: $t+k]$ )

if(PRT|q].machine_no $[k \mid]==$ presen!_mac)

:

numb $=1$;

next_mac $=\mathrm{P}^{\prime} R T[q]$ machine_no $\left.[k]+1\right]$ :

Whilerbuffer[ nexi_mac] queue[numb] :=(0)

!

waiting_job $=$ buffer[nexi_mac].queue[numb]:

workinqueue $=$ workinqueue + PRT[waiting_job].operation[next_mac];

t+numb: ;

workinqueue $=$ workinqueue + PRT[q].operation $[$ PRT[q].machine_no[k] ] ;

return(workinqueue);

;

else

$\operatorname{retum}(0)$

;

siruct bufs lwkr_buf(bufno)

int bufno;

\{

\#define FALSE 0

\#define TRUE 1

int $j$, sorted = FALSE:

int temp:

int $\mathrm{n}=1$;

while(buffer[bufno].queue[n] !=0)

$++n$;

$-n$;

while (!sorted)

\{

sorted $=$ TRUE

for $(j=1 ; j<n ;++j)$

if (PRT[buffer[bufno].queue[i]].operation[0] > PRT[buffer[bufno].queue[j+]]].operation[0])

3

temp = buffer[bufno].queue[i];

buffer[bufno].queue[j]=buffer[bufno].queue $[j+]$;

buffer[bufno].queue[j+1]=temp;

;

sorted = FALSE;

retum;

I 


\section{Appendix A3: Comparison of NN results with other dispatching rule results}

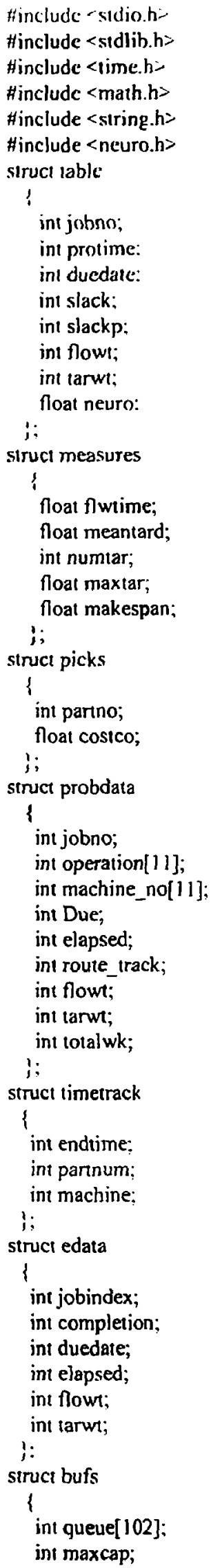


float LOAD[ ] I].PLOAD[1] ], maxioad:

struct inc’asures $M M[10]$ :

struct bufs buffer $[21]$ :

struct timetrack cevent|211]:

struct colata sink[1]1]:

struct probdata PRT|101]:int dispatching_rulc[16]:

int rulesel.consel;

int TIMENOW, dd,bst:

float Tardiness. TTard.Bcost.CCost:

int Count=(), machines.h.v:

char ncat[3]:

int droutput|31]:

main(void)

!

FILE * sinput,*soutput:

struct tablespt_sort().edd_sort().mslk_sort().Ipt_sort().mslp_sort(),wspt_sort();

struct table clear_job().update_jobs().random sort();

struct measures calculate_measures().calculate_cost().schedule_costs():

struct edata find_sched():

struct bufs

sp!_buf(),lpt_buf().1w'spt_buf().CD().tsp!_buf().edd_buf(),slack_buf(),covert_buf(),mdd_buf().lwkr_buf(),twk_buf(); struct bufs snq_buf(),awinq_buf().append_to_bufier(),unload_machine();

int combox. 244$][6], \mathrm{kl}, \mathrm{k} 2, \mathrm{k} 3, \mathrm{k} 4, \mathrm{k} 5, \mathrm{jj}, \mathrm{combination:}$

float mc square[1]], maxvar.makespans.flowtimes:

float besi_result,performance;

int remove_job(),max_prot(), max_date,member_of(), Avertot;

float Mflow,Bflow;

double n_vector[16],n_output[16].maxout:

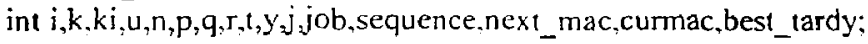

int place. found;

int best_tardiness,best_flow,best_combo.mac_candidate[14],mincostjob,totpro,mincostdue,mondue;

int BEST[300][11], njobs[3];

int greater_of().counter,tie_counter:

char FFname[ 12],WWR[4 $\overline{4}]$,dest2[30], dest ] [30].dest[30], F dat[ 12];

char ${ }^{*}$ str1;

int combocounter $=0$;

if $(($ sinput $=$ fopen $(" c:$ iltcllbin ljobshopslldatailmultest.dat","r") $)==$ NULL $)$

$\{$ fprintf(stderr, "Cannot open output file . In"); return 1;

;

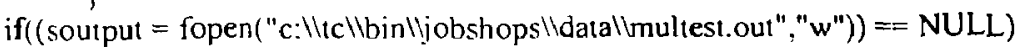

$\{$ fprintf(stderr,"Cannol open output file . $\backslash n ") ;$

return 1;

;

printf("Select Dispatching Rule Policy : $\ln (n ")$;

printf("1. SPT for all machines in");

printf("3. LWKR for all machines $(n ")$;

printf("2. LPT for all machines $\backslash n "$ );

printf("4. Veural Selection $\backslash n ")$;

scanf("\%d",\&rulesel):

if(rulesel != 1 \&\& rulesel !=2 \&\& rulesel !=3 \&\& rulesel !=4)

$\{$ printf("Leaving the program . $(n$ "): return 1;

if(rulesel $\stackrel{?}{=}=1)$

for $(k=1: k<=$ machines; $++k)$ dispatching rule $[k]=1$;

if $($ rulesel $==2$ )

for $(k=]: k<=$ machines: $++k)$ dispatching_rule $[k]=2$;

if (rulesel $==3$ )

for $(k=l: k<=$ machines: $++k)$ dispatching_rule $[k]=3$;

makespans $=$ flowtimes $=0$;

while(!feof(sinput))

\{ 
fscanf( sinput." "\%d \%d".\&Count.\&machines):

if(teoff sinpul))

break:

for $(k=0) ; k<=10 ;++k)$ buffer $\mid k]$ maxcap $=0$ :

for $(k=0): k r=15 ;++k)$

ispatching_rule $[k]=0 ; n_{-}$vector $[k]=(j .0 ;:$

for $(k=1: k<=$ machines: $++k)$

dispatching rulc $[\mathbf{k}]=$ rulese $:$

for $(j=1: i c=210:++i)$

event $[i] \cdot$ endtime $=$ event $[i] \cdot$ parnum $=$ event $[i] \cdot$ machine $=0$;

for $(i=1: j=10 ;++i)$

$\operatorname{for}(j=0 ; j==[0]:++i)$

buffer $[i]$.queue $[\mathrm{j}]=0$ :

for $(j=1 ; j<=11(j ;++i)$

$\sin k[i] \cdot j$ ohindex $=\sin k[i] \cdot \operatorname{completion}=\sin k[i] \cdot$ duedate $=\sin k[i] \cdot$ elapsed $=0$;

for $(i=0) ; i<=$ Count $;++i)$

$\operatorname{for}(k=0: k<=10 ;++k)$

$\left\{\right.$ PRT[i].operation $[k]=0: P R T[i]$.machine_no[k] $=0 ; \mathrm{mc}_{-}$square $[\mathrm{k}]=0 ;$;

for $(i=1 ; i<=$ Count $;++i)$

i

totpro $=0$

for $(k=1 ; k<=$ machines; $++k)$

fscanf(sinput,"\%d ".\&.PRT[i].machine_no[k]);

;

for $(k=1 ; k<=$ machines; $++k)$

i

fscanf(sinput,"\%d ".\&PRT[i].operation[PRT[i].machine no[k]]);

totpro $=$ torpro + PRT $[\mathrm{i}]$.operation[PRT[i].machine_no[k]]; ;

PRT[i].operation $[0]=$ totpro;

PRT[i].totalwk = totpro:

PRT[i].jobno $=\mathrm{i}$;

PRT[i].elapsed $=0$;

PRT[i].route_track $=0$;

PRT[i].flowt $=1$;

PRT[i].tanwt $=1$;

fscanf(sinput," \%d".\&PRT[i].Due);

for $(k=1: k<=$ machines; $++k)$

$\mathrm{LOAD}[\mathrm{k}]=0$;

for $\left(k=1 ; k<=\right.$ machines: $\left.{ }^{++k}\right)$

for $(i=1: i<=$ Count $+++i)$

maxload $=0$.

LOAD $[k]=\mathrm{LOAD}[\mathrm{k}]+$ PRT[i].operation[k];

for $(k=1 ; k<=$ machines: $++k)$

if(LOAD $[k]>$ maxload) maxload = LOAD $[k]$ :

for $(k=1: k<=$ machines: $++k)$

$\max$ var $=0.0$ :

$n \_$vector $[k]=\operatorname{LOAD}[k] /$ masload;

for $(k=1: k<=$ machines; $++k)$

for $(i=1 ; i<=$ Count; $++i)$

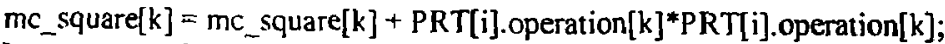

$m c$ square $[k]=\left(m c_{\text {__square }}[\mathrm{k}]-\mathrm{LOAD}[\mathrm{k}]^{*} \mathrm{LOAD}[\mathrm{k}] /\right.$ Count $) /($ Count-1);

if(mc_square $[\bar{k}]>$ maxvar) maxvar $=m c_{-}$square $[k]$;

(

for $(k=$ machines $+1 ; k<=2 *$ machines $;+k)$

$n_{-}$vecior $[k]=m c_{-}$square $[k-$ machines $] /$ maxvar;

for $(k=1 ; k<=$ machines; $++k)$

\{

place $=0$; 


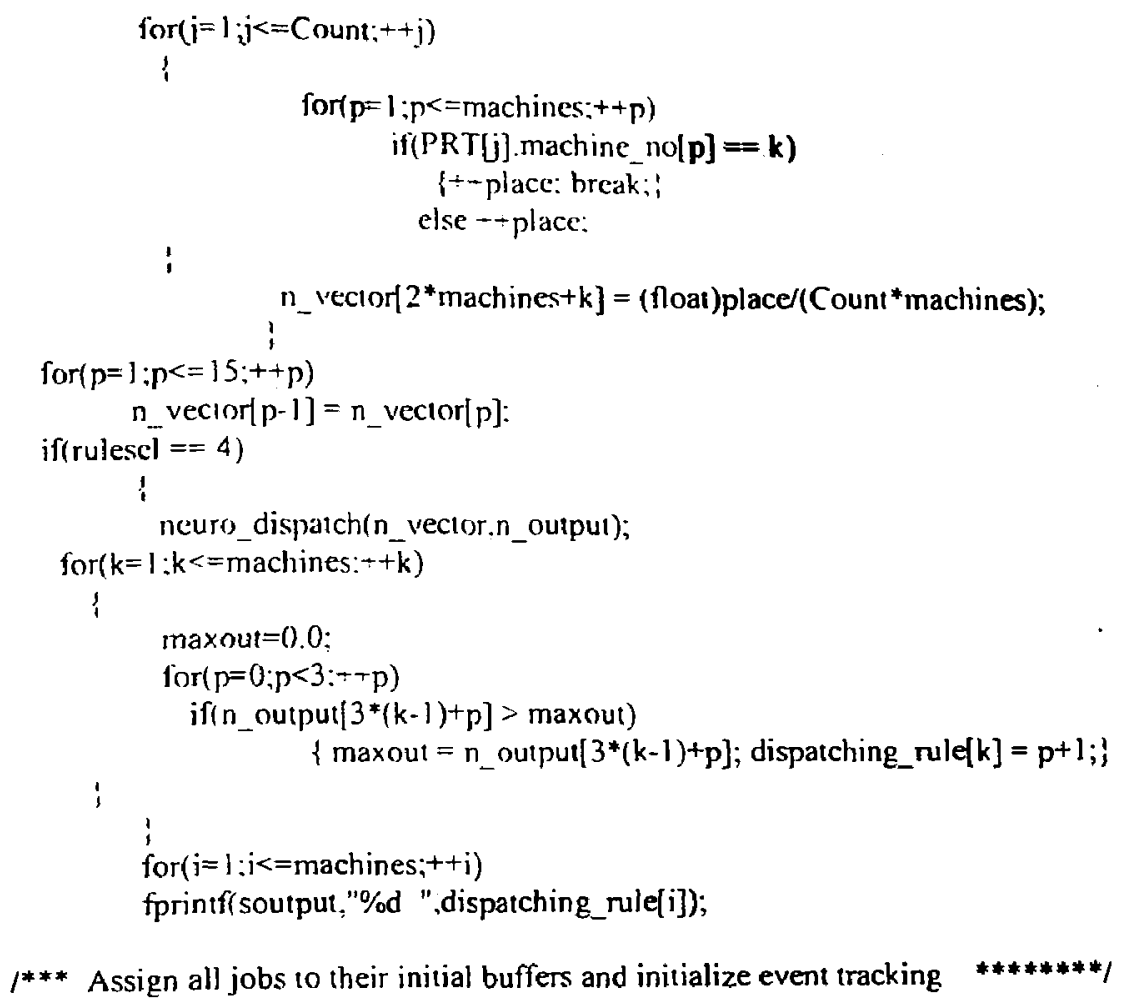

/*** Assign all jobs to their initial buffers and initialize event tracking

\section{Refer Appendix A2}




\section{Appendix A4: Test Problem generation}

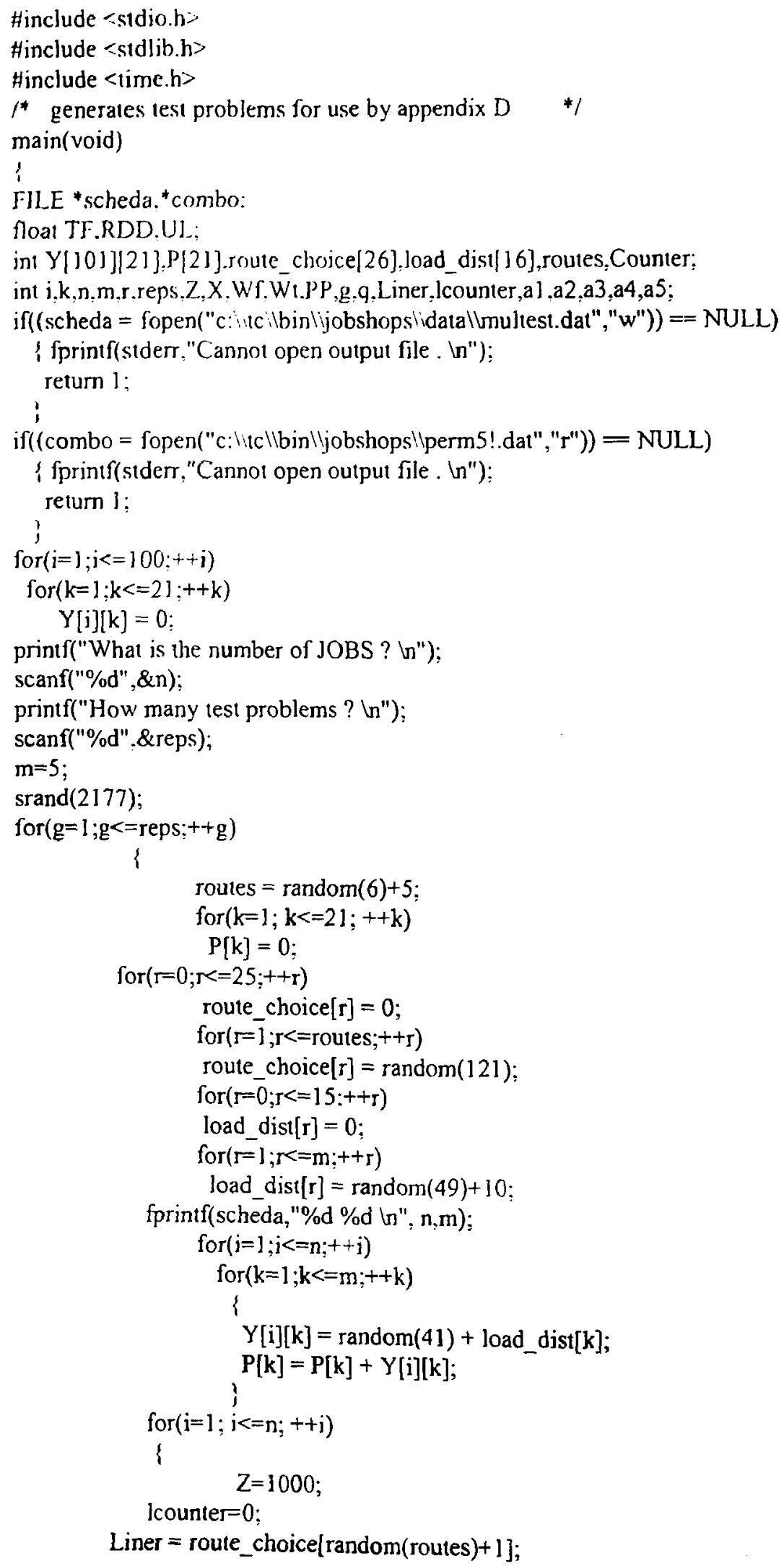


while( Liner $!=$ Icounter)

! fscanf(combo."\%d \%d \%d \%d \%d $\backslash n$ ".\&al,\&a2.\&a3.\&a4.\&a5);

++ lcounter; ;

fprintr(scheda,"\%d \%d \%d \%d \%d ".al.a2,a3,a4,a5);

rewind (combo):

fprintf(scheda,"\%d ",Y[i][a] ]);

fprintf(scheda,"\%d ",Y[i][a2]);

fprintf(scheda,"\%d ",Y[i][a3]);

fprintf(scheda,"\%d ",Y[i]|a4]);

fprintf(scheda,"\%d ",Y[i][a5]):

fprintf(scheda,"lt \%d ",Z);

fprintf(scheda:" $" n ")$;

;

fprintf(scheda,"lnın");

++ Counter;

了

printf("counter iss : \%dhn",Counter);

fclose(combo);

fclose(scheda);

j 
Appendix B: Arena simulation model

File Edit View Iools Aronge Medule Bum window Help

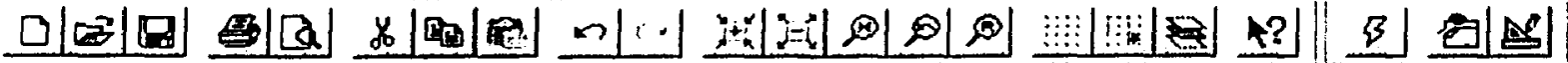

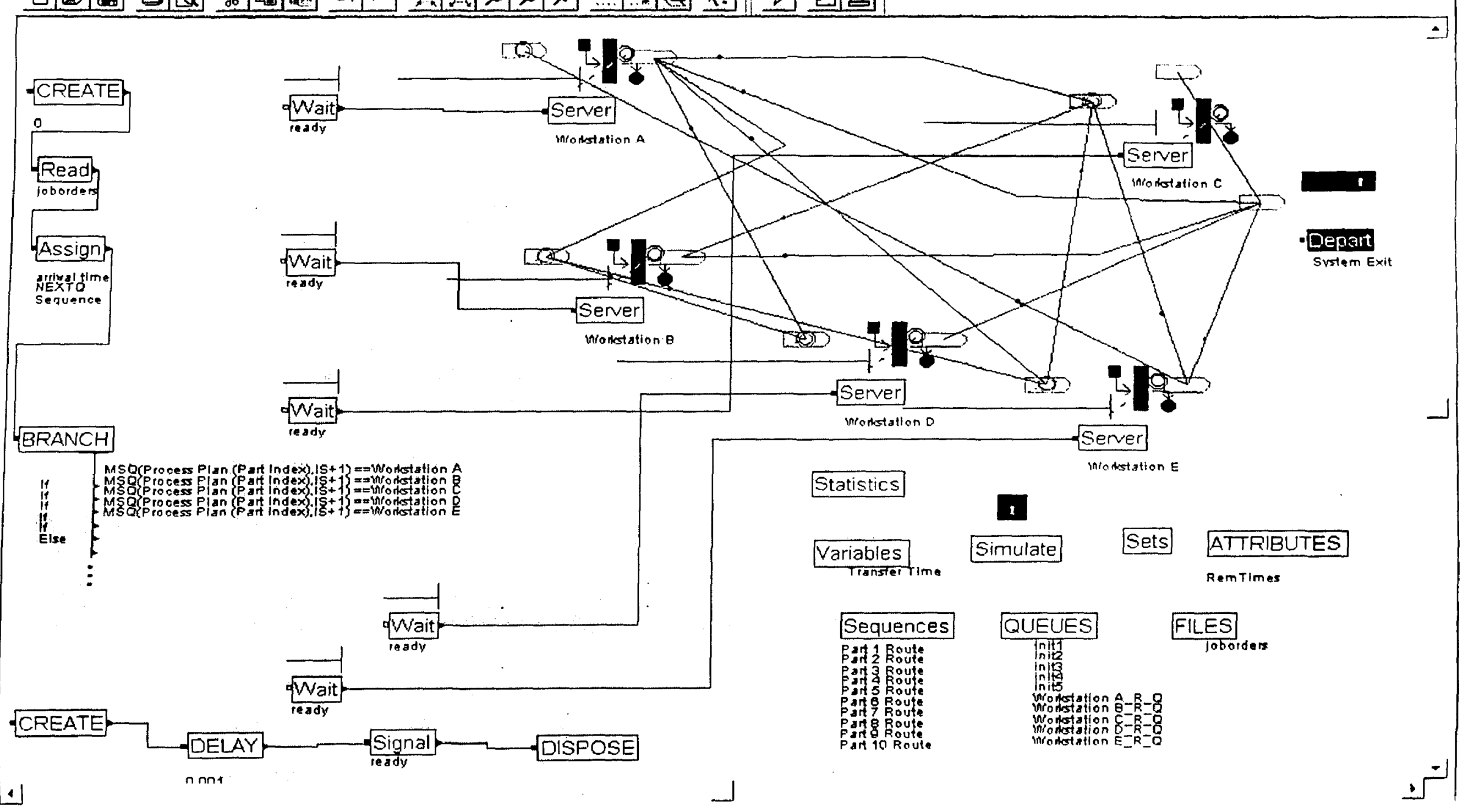


Appendix $\mathrm{C}$ : Determining number of hidden neurons for performance objective of Minimizing Makespan

\begin{tabular}{|c|c|}
\hline Number of hidden layer & 3 \\
Learning rate & 0.1 \\
Momentum & 0.1 \\
Initial Weights & 0.3 \\
Pattern Selection & Random \\
Weights updates & Vanilla \\
Number of training patter & 2494 \\
Number of test & 1200, combination of $\mathbf{n}=10,15,20$ \\
Number of hidden neurons & 6 to 18 \\
\hline
\end{tabular}


Appendix C2: Determining number of hidden neurons for performance objective of Minimizing Mean Flowtime

\begin{tabular}{|c|c|}
\hline Number of hidden layer & 1 \\
Learning rate & 0.05 \\
Momentum & 0.05 \\
Initial Weights & 0.3 \\
Pattern Selection & Rotational \\
Weights updates & Turboprop \\
Number of training patter & 2636 \\
Number of test & 1200, combination of $n=10,15,20$ \\
Number of hidden neurons & 10 to 25 \\
\hline
\end{tabular}




\section{Appendix D1: Neural Weights for Makespan network}

$I^{*}$ Inser this code ina your C program to fire the C:WSHELL2ISPT\&LPTd.MWKR network *I

$I^{*}$ This code is designed to be simple and fast for poning to any machine */

$t^{*}$ Therefore all code and weights are inline without looping or data storage *

* which mighı be harder to por between compilers. */

Hinclude <math.h>

void neuro_dispatch(double *inarray, double *outarray)

!

double netsum:

double featurc2[9]:

$1 *$ inarray $[0]$ is total processing time on machine $1 * 1$

$1^{*}$ inartay 1 ] is total processing time on machine $2 * /$

$1^{*}$ inarray 2$]$ is total processing time on machine $3 * i$

$I^{*}$ inarray[3] is total processing time on machine $4 *$ /

$1 *$ inarray[4] is tolal processing time on machine $5 * /$

$1^{*}$ inarray $[5]$ is variance processing time on machine $1 *$

$1 *$ inarray $[6]$ is variance processing time on machine $2 * /$

$/ *$ inarray[ 7$]$ is variance processing time on machine $3 * /$

$/ *$ inarray $[8]$ is variance processing time on machine $4 * /$

$I^{*}$ inarray $[9]$ is variance processing time on machine $5 * /$

$/ *$ inarray $[10]$ is mean routing order of machine $1 * /$

$l^{*}$ inarray [ $[1]$ is mean routing order of machine $2 * /$

$1 *$ inarray $[12]$ is mean routing order of machine $3 * /$

$l^{*}$ inarray $[13]$ is mean routing order of machine $4 * t$

$/ *$ inarray[ [4] is mean routing order of machine $5 * /$

$l^{*}$ outarray $[0]$ is SPT on machine $] * /$

$1 *$ outarray[ ]] is LPT on machine $1 * /$

$l^{*}$ oularray[2] is MWKR on machine 1*/

$l^{*}$ outarray [3] is SPT on machine $2 * /$

$I^{*}$ outarray [4] is LPT on machine $2 * /$

$1 *$ outarray[5] is MWKR on machine $2 *$

$l^{*}$ outarray[6] is SPT on machine $3 * /$

$1 *$ outarray[7] is LPT on machine $3 * /$

/* outarray[8] is MWKR on machine $3 * /$

$l^{*}$ outartay[9] is SPT on machine $4 * /$

$/ *$ outarray[ ]0] is LPT on machine $4 * /$

$l^{*}$ outarray[ [1] is MWKR on machine $4 * /$

$l^{*}$ outarray[ 12] is SPT on machine $5 * /$

$1 *$ outarray [ 13] is LPT on machine $5 * /$

$l^{*}$ outarray[ 14] is MWKR on machine $5 * /$

inarray $[0]=1.0 /(1.0+\exp (-($ inarray $[0]-.8072053) / .1779804))$ :

inarray $[1]=1.0 /(1.0+\exp (-($ inarray $[1]-.7982979) / .1812125))$ :

inarray[2] $=1.0 /(1.0+\exp (-($ inarray $[2]-.8054764) / .1795151))$ :

inarray $[3]=1.0 /(1.0+\exp (-($ inarray $[3]-.8004816) / .1790274))$ :

inarray $[4]=1.0 /(1.0+\exp (-($ inarray $[4]-.8064976) / .1814937))$

inarray $[5]=1.0 /(1.0+\exp (-($ inarray $[5]-.771103) / .1943457))$ :

inarray $[6]=1.0 /(1.0+\exp (-($ inarray $[6]-.7586824) / .1922863))$ :

inarray $[7]=1.0 /(1.0+\exp (-($ inarray $[7]-.7655738) / .1942723))$ :

inarray $[8]=1.0 /(1.0+\exp (-($ inarray $[8]-.769994) / .1929265))$; 


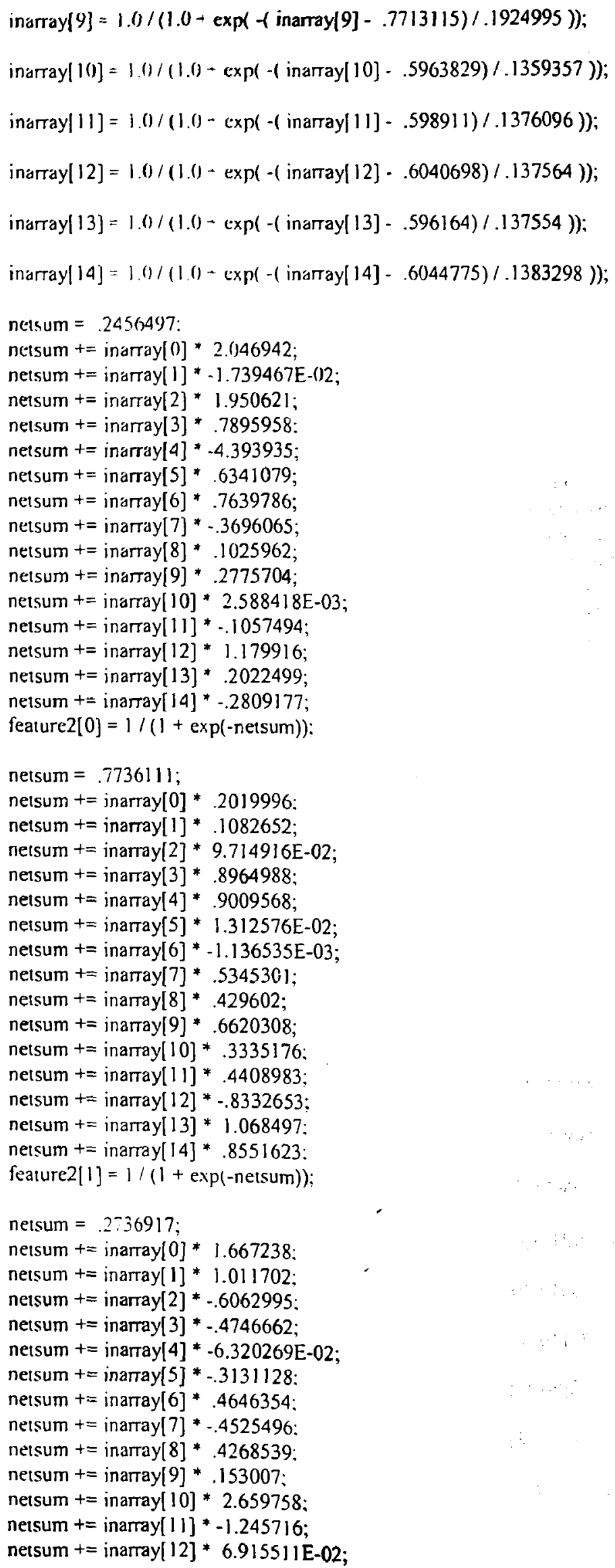


netsum + = inarray[13] * - 1.128644; netsum $+=$ inarray[ 14] * 1.168092;

featurc2[2] $=1 /(1+\exp (-$ netsum $))$ :

netsum $=.4272505$;

netsum $+=$ inarray[0] * .7500188;

netsum $+=$ inarray[ 1] * 1.000085 ;

netsum $+=$ inarray[2] * - 1.751807;

netsum $+=$ inarray[3] * .2685844;

netsum += inarray[4] * 1.027902;

netsum $+=$ inarray [5] * .6474725;

netsum $+=$ inarray[6] * .1521454:

netsum $+=$ inarray[7] * .2068681:

netsum $+=$ inarray $[8] *-.5180956$;

netsum += inarray[9] * .1931846:

nctsum $+=$ inarray[10] * -1.069892 ;

netsum += inarray[11] * .6269137;

netsum $+=$ inarray [12] * 1.828143 ;

netsum $+=$ inarray [13] * - . 174372 ;

netsum $+=$ inarray [ 14] * .3147894;

feature2[3] $=1 /(1+\exp (-$ netsum $))$;

netsum $=1.253435$;

netsum $+=$ inarray [0] * .4281614;

netsum $+=$ inarray [1] * .263829;

netsum + = inarray[2] * .7757708;

netsum += inarray[3] * .8198949;

netsum $+=$ inarray [4] * .4531571;

netsum $+=$ inarray $[5] * .7415228$;

netsum $+=$ inarray [6] * .3905536 ;

netsum += inarray[7] * .5716818;

netsum $+=$ inarray $[8] * .5664634$;

netsum $+=$ inarray [9] * .4108108;

netsum $+=$ inarray $[10] * 1.020716$;

netsum $+=$ inarray [11] * .6482075;

netsum $+=$ inarray [12] * .3916479;

netsum $+=$ inarray [13] * .82701;

netsum $+=$ inarray [14] * .5516309;

feature $2[4]=1 /(1+\exp (-$ netsum $))$;

netsum $=1.020303$;

netsum $+=$ inarray $[0] * .6176658$;

netsum $+=$ inarray $[1] *-.1641615$;

netsum $+=$ inarray [2] * .6528724;

netsum $+=$ inarray[3] * .5118068;

netsum $+=$ inarray [4] * 1.187099 ;

netsum $+=$ inarray [5] * .357643;

netsum $+=$ inarray $[6] * .5779907$;

netsum $+=$ inar:ay[7] * .6766816;

netsum $+=$ inarray $[8] * .337665$;

netsum $+=$ inarray[9] * .5777485 ;

netsum $+=$ inarray[ 10$] *$ 0 * .7961912;

netsum $+=$ inarray [1 1] * .6893328;

netsum $+=$ inarray [12] * .5017431;

netsum $+=$ inarray $[13] *-.2587531$;

netsum $+=$ inarray [14] * .545307;

feature $2[5]=1 /(1+\exp (-$ netsum $))$;

netsum $=-.857754$

netsum $+=$ inarray $[0] *-.7458848$;

netsum $+=$ inarray $[1] *-1.533975$;

netsum $+=$ inarray[2] * -1.908274 ; 
netsum $\rightarrow=$ inamay|3] * 0.414888 :

netsum t= inarrayl4] * - 1.083793:

neisum t = inarrayls] * -.1197545 :

nctsum $+=$ inarraylo] $* .1958(144$ :

netsum += inaray|7] * .4577059;

netsum += inartay| 8 ] * -.8148838 ;

netsum $+=$ inarray|9] * -.3346533 ;

netsum + = inarray 1 10] * -.4140265:

netsum += inarray[ 11] * -.9390895 :

netsum $+=$ inarray[12] *.1293133:

netsum $+=$ inarray 13] * -.2089598 :

netsum $+=$ inarray $\mid 14$ ] * $-.150(0)(09$;

feature $2[6]=1 /(1+\exp (-n e t s u m))$ :

netsum $=.9072358$;

netsum $+=$ inarray[(0) * -4.788719 ;

netsum $+=$ inarray $[1] * 1.544868$ :

netsum $+=$ inarray [2] * 2.137923;

netsum $+=$ inarray $[3] * .6501429$;

netsum $+=$ inarray[4] * 1.509744;

netsum $+=$ inarray $[5] * .552145$;

netsum $+=$ inarray[6] * .7359388 ;

netsum $t=$ inarray[7] * -.3814477 ;

netsum $+=$ inarray $[8] * .207289$;

netsum $+=$ inarray[9] * -.3526275 ;

netsum $+=$ inarray [10] * .7480308;

netsum $+=$ inarray[11] $*-.7977181$;

netsum $+=$ inarray [12] * 1.289536;

netsum $+=$ inarтay[13]* .1694474;

netsum $+=$ inarray $[14]$ * -.1155627 ;

feature $2[7]=1 /(1+\exp ($-netsum $))$;

netsum $=.2858913$;

netsum $+=$ inarray[0] * -1.584813 ;

netsum $+=$ inarray[ 1] * 3.49939;

netsum $+=$ inarray [2] * -1.439081 ;

netsum $+=$ inarray [3] * .1945082;

netsum $+=$ inarray $[4] *-2.07647$;

netsum $+=$ inarray[5] * $-6.070224 \mathrm{E}-03$;

netsum $+=$ inarray[6] * -.3148423 ;

netsum $+=$ inarray[ 7] * .3856115;

netsum $+=$ inarray[ 8$] *-.1825979$;

netsum $+=$ inarray [9] * .2919024;

netsum $+=$ inarray $[10] *-.3072858$;

netsum $+=$ inarray $[11] * 1.34602$;

netsum $+=$ inarray $[12] *-.3143554$;

netsum $+=$ inarray[13] * -.6019555 ;

netsum $+=$ inarray $[14] *-.4247503$;

feature $2[8]=1 /(1+\exp (-$ netsum $))$;

netsum $=-.3141774$;

netsum $+=$ feature $2[0] * .238933]$;

netsum $+=$ feature $2[1] *-.54418$;

netsum $+=$ feature2[2] * -1.70218 ;

netsum $+=$ feature2 $[3] * 1.199972$;

netsum $+=$ feature2[4] * -.4256195 ;

netsum $+=$ feature2[5] * -.2628123;

netsum $+=$ feature2[6] * .19171116;

netsum $+=$ feature2[7] * 1.139177 ;

netsum $+=$ feature $2[8] * .2864685$;

outarray $[0]=1 /(1+\exp (-n e t s u m)) ;$ 


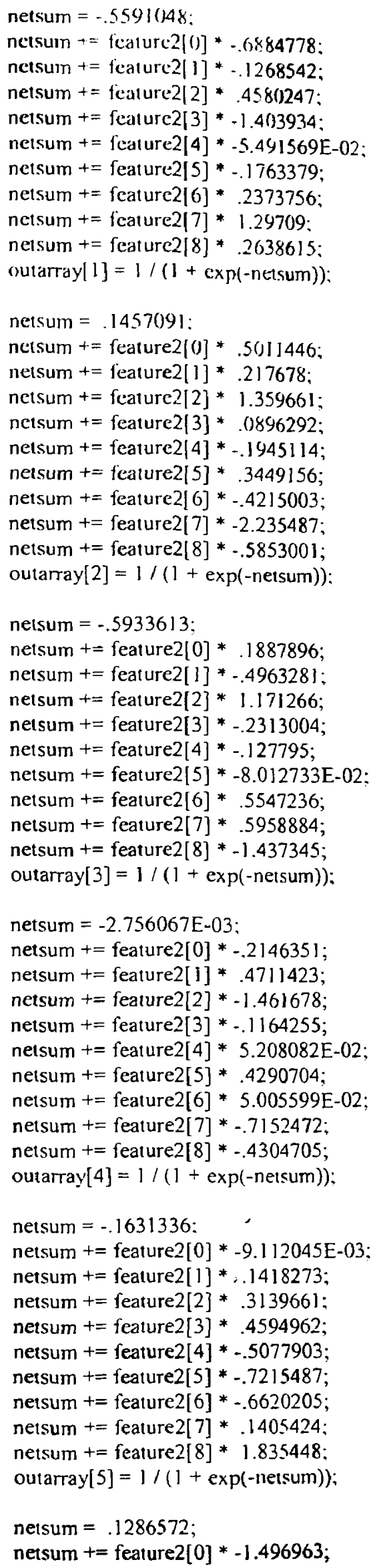


netsum $+=$ featurc2[1] * .6675627;

netsum $+=$ feature2[2] *3.467289E-02:

nctsum $\rightarrow=$ feature2[3] *.211388:

netsum $*=$ feature2 $[4] * .4022121$;

netsum $+=$ featurc2 $[5] * 9.279378 \mathrm{E}-02$;

netsum $+=$ feature2[6] * .221469:

ne1sum $+=$ feature2 27] $*-1.08096$;

netsum $\rightarrow=$ feature2 $[8] * .5696868$;

outarray $[6]=1 /(1+\exp (-$ netsum $))$ :

netsum $=-.307(033$

nctsum $=$ featurc2[0]* $-.245(1) 359$ :

netsum $+=$ feature2 [1] * -1.0577()1:

nctsum $=$ f caturc2[2] * .643006;

netsum $\Rightarrow$ feature2[3] *1.318899;

netsum $+=$ feature $2[4]^{*}-.593664$;

nelsum $+=$ feature2[5] * -.3161325;

netsum $+=$ feature2[6] * .6486963;

netsum $+=$ feature2[7] * -.2953574 ;

netsum $+=$ fearure $2[8] * .2141867$;

outarray $[7]=1 /(1+\exp (-$ netsum $))$;

nctsum $=-2.572132 \mathrm{E}-03$;

netsum $+=$ feature2[0] * 1.719462;

netsum $+=$ feature2[1]* -.3831635 ;

netsum $+=$ feature2[2] * -.6865217;

netsum $+=$ feature2[3] * -1.433495;

netsum $+=$ feature2[4] * -.1088401 ;

netsum $+=$ feature2[5] * $1.501535 \mathrm{E}-02$;

netsum $+=$ feature2[6] * -.8874764 ;

netsum $+=$ feature2[7] * 1.299559;

netsum $+=$ feature $2[8] *-.7590157$;

outarray $[8]=1 /(1+\exp (-$ netsum $))$;

netsum $=-.3838195$;

netsum $+=$ feature2[0] * -.1236574 ;

netsum $+=$ feature2[ 1] * -.9973538 ;

netsum $+=$ feature2[2] * .7501443;

netsum $+=$ feature2[3] * 1.06568;

netsum $+=$ feature2[4] * -.3194561 ;

netsum $+=$ feature2[5] * .4040247;

netsum $+=$ feature2[6]*-1.106399;

netsum $+=$ feature2[7] * -.2665701 ;

netsum $+=$ feature2 $[8] * .2000748$;

outarray $[9]=1 /(1+\exp (-$ netsum $))$;

netsum $=.253037$

netsum $+=$ feature2[0] * .0630246;

netsum $+=$ feature2[1] * .3430368:

netsum $+=$ feature $2[2] *-.9319632$;

netsum $+=$ feature2[3] * -.5230323 ;

netsum $+=$ feature2[4] * .1765063;

netsum $+=$ feature2[ 5 ] * -.2045487 ;

netsum $+=$ feature2[6] $*-1.250098$;

netsum $+=$ feature2[7] * 4.259654E-02;

netsum $+=$ feature2[8] * -.1516763;

outarray $[10]=1 /(1+\exp (-$ netsum $))$;

netsum $=-.5481886$;

netsum $+=$ feature2[0] * .1021582;

netsum $+=$ feature2[1] * .2943085 ;

netsum $+=$ feature2[2] * .166375; 


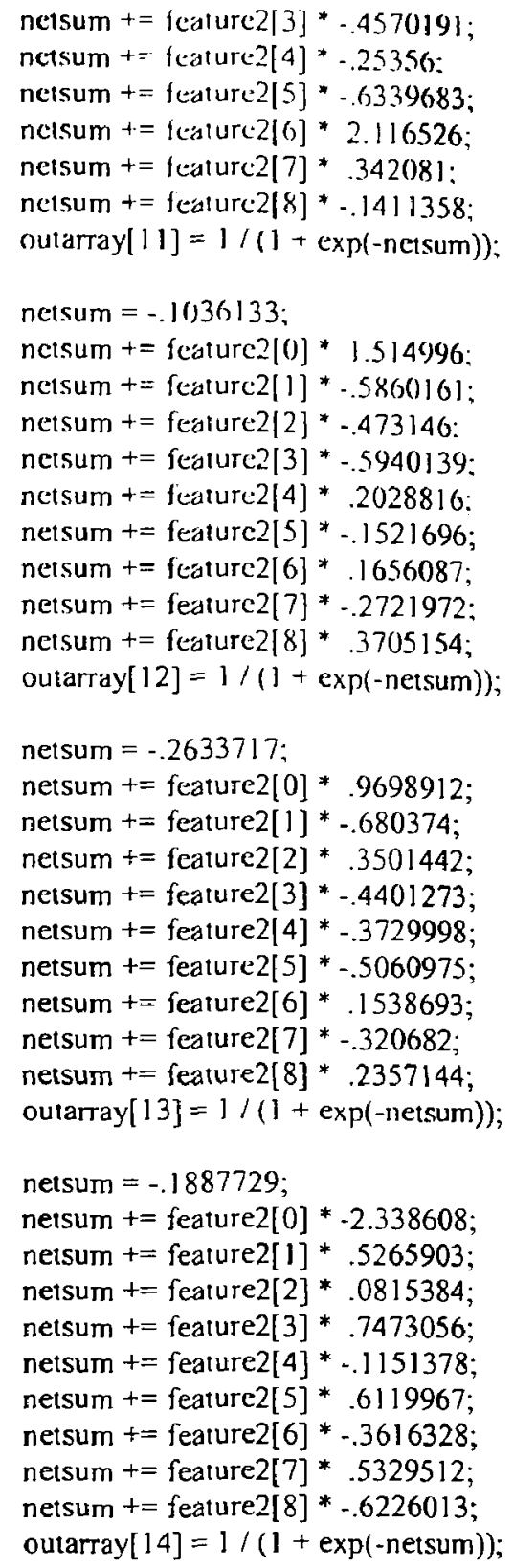




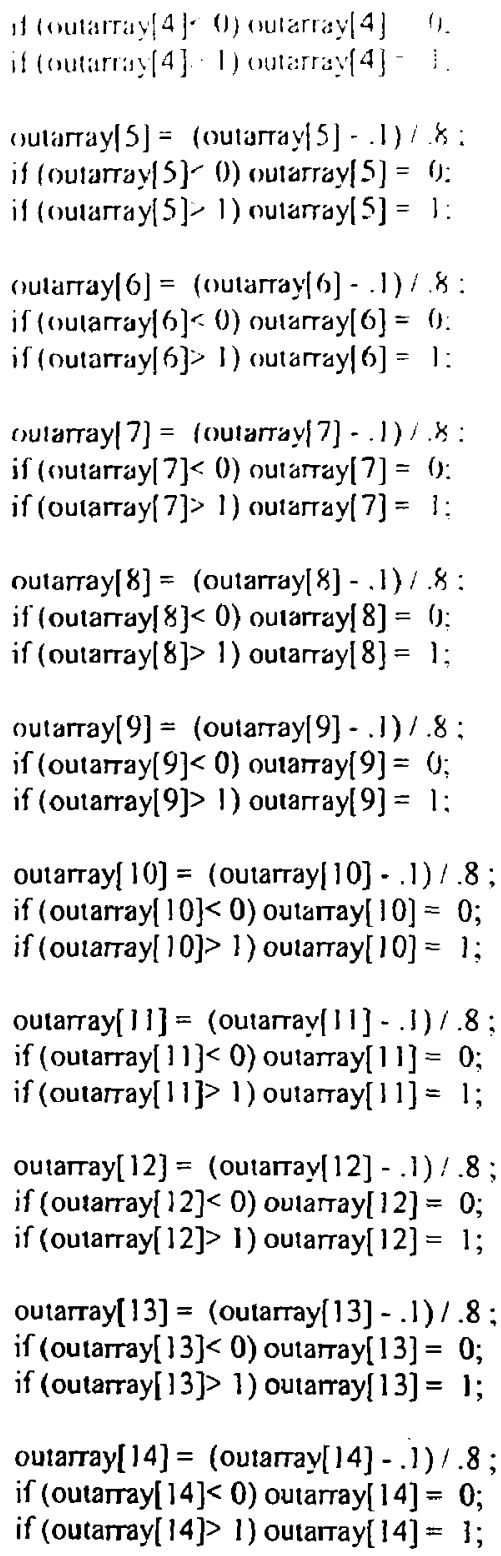




\section{Appendix D2: Neural Weights for Mean Flowtime network}

/* Insert this code into vesur C program of fire the C:NSHELL2'SPT\&PTWINQ\&LWKR network *

$1^{*}$ This code is designed 10 be simple and fast for poring to any machine*!

1* Therefore all code and weights are inline without lonping or data storage *I

1* which might be harder to port between compilers. *I

\#include - math.h>

void neuro_dispatch(double *inarray, double *oularray)

?

double netsum:

double feature2[20]:

$l^{*}$ inarray $[0]$ is $B * /$

$l^{*}$ inarrayl 1$]$ is $C * /$

$f *$ inarray[2] is $D * /$

$/$ inarray [3] is $E * /$

$/$ * inarray[4] is $F * /$

$f^{*}$ inarray [5] is $\mathrm{G} * /$

$/$ * inarray[6] is $\mathrm{H} * /$

$1 *$ inarray [7] is I *1

$l^{*}$ inarray[ 8$]$ is $\mathrm{J} * l$

$l *$ inarray [9] is $K * /$

$/ *$ inarray $[10]$ is $L$ */

$/ *$ inartay[11] is $M * /$

$/$ * inarray [12] is $\mathrm{N} * /$

$I^{*}$ inarray [13] is $\mathrm{O}$ */

$f^{*}$ inarray [14] is $\mathrm{P} * /$

$I^{*}$ outarray[0] is $\mathrm{Q}^{*} /$

$l^{*}$ outarray[1] is $\mathrm{R} * /$

$I^{*}$ outarray[2] is $S * /$

$I^{*}$ outarray[3] is T*/

$I^{*}$ outarray[4] is $U *$

/* outarray[5] is $V * /$

$/ *$ outarray[6] is $\mathrm{W} * /$

$/ *$ outarray[7] is $X * 1$

$l^{*}$ outarray [8] is $Y^{*} /$

$I^{*}$ outartay[9] is $Z * /$

$l^{*}$ outarray [10] is AA */

$I^{*}$ outarray[11] is $A B * 1$

/* outarray[ [2] is $\mathrm{AC} *$ /

$1^{*}$ outarray[ ] 3] is AD */

$l^{*}$ outarray[ [4] is AE */

if (inarray $[0]<.33)$ inarray $[0]=.33$ : if (jnarray $[0]>1)$ inarray $[0]=1$;

inarray $[0]=($ inarray $[0]-.33) / .67$;

if (inarray $[1]<.31$ ) inarray [1] = .31; if (inarray[ 1$]>1$ ) inarray [1] = 1 ;

inarray[1] $=($ inarray[1] -.31$) / .69$;

if (inarray[2]<.32) inarray[2] $=.32$; if (inarray[2]> 1) inarray[2] = 1 ;

inarray[2] $=($ inarray $[2]-.32) / .68$ :

if (inarray[3]<.31) inarray[3] = .31; if (inarray [3]> 1) inarray [3] $=1$;

inarray $[3]=($ inarray $[3]-.31) / .69$;

if (inarray $[4]<.34)$ inarray $[4]=.34$; 
if (inarray[4] = 1) inarray[4] = 1: inarray $[4]=($ inarray $[4]-.34) / .66$;

il (inarray $[5]<.49)$ inarray $[5]=.49$; if ( inarray|5]> 1) inarray[5] = 1; inarray $[5]=($ inarray $[5]-.49) / .51$;

if (inarray $[6]<.51$ ) inarray $[6]=.53$; if (inarray[6] $>1$ ) inarrayl6] $=1$ : inarrayl6] $=($ inarray $[6]-.51) / .49$;

if ( inarray [7]<.53) inarray $[7]=.53$ : if (inarray| 7] $>1$ ) inarray[7] $=1$; inarrayl 7$]=($ inarray $[7]-.53) / .47$;

if (inarray $[8]<.55)$ inarray $[8]=.55$; if (inarray [8]> 1) inarray [8] = 1 ; inarray $[8]=($ inarray $[8]-.55) / .45$ :

if ( inarray $[9]<.57)$ inarray $[9]=.57$; if ( inarray[9]> 1) inarray[9] = 1; inarray $[9]=($ inarray $[9]-.57) / .43$;

if (inarsay[ 10$]<.22)$ inarray $[10]=.22$; if (inarray[ 10]> .93) inarray[ 10$]=.93$; inarray $[10]=($ inarray $[10]-.22) / .71$;

if (inarray $[11]<.25)$ inarray $[11]=.25$ if (inarsay[ $[1]>.92)$ inarray [ 11$]=.92$; inarray $[1]]=($ inarray $[11]-.25) / .67$;

if (inarray [ 12]<.22) inarray[ 12] = .22; if ( inarray[ 12]> .96) inarray[ 12] = .96; inarray $[12]=($ inarray $[12]-.22) / .74$;

if ( inarray[ 13]<.22) inarray[ 13] = .22; if (inarray[13]> .95) inarray[13] = .95; inarray $[13]=($ inarray $[13]-.22) / .73$;

if (inarray[ 14]<.23) inarray[ 14] = .23; if ( inarray[ 14]> .96) inarray[ 14] $=.96$; inarray $[14]=($ inarray $[14]-.23) / .73$;

netsum $=-2.127025$;

netsum $+=$ inarray $[0] *-1.534661$ :

netsum $+=$ inarray $[1]$ * 1.385606 :

netsum $+=$ inarray [2] * - - .67123:

netsum $+=$ inarray [3] * -.2299798 :

netsum $+=$ inarray[4] * 5.66486:

netsum $+=$ inarray [5] * -.4372981 ;

netsum $+=$ inarayl6] * -.5978695 :

netsum $+=$ inarтay [7] * -.5532722 ;

netsum $+=$ inarray $[8] *-.488466]$;

netsum $+=$ inarray $[9] *-.6174343$;

netsum $+=$ inartay [1 0$] *-.3179735$;

netsum $+=$ inarray [ 11$] *-.7406918$;

neisum $+=$ inarray [12] * -.3327855 ;

netsum $+=$ inarray [13] * -.5212398 ;

netsum $+=$ inarray [14]*-1.144423;

feature2[0] = $1 /(1+\exp (-$ netsum $))$;

netsum $=.1270918$; 


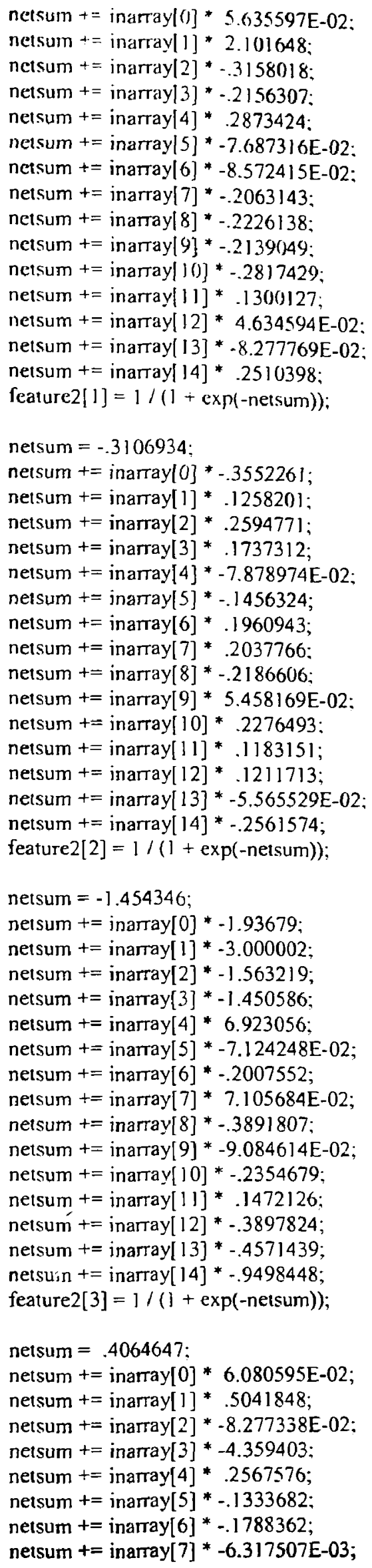




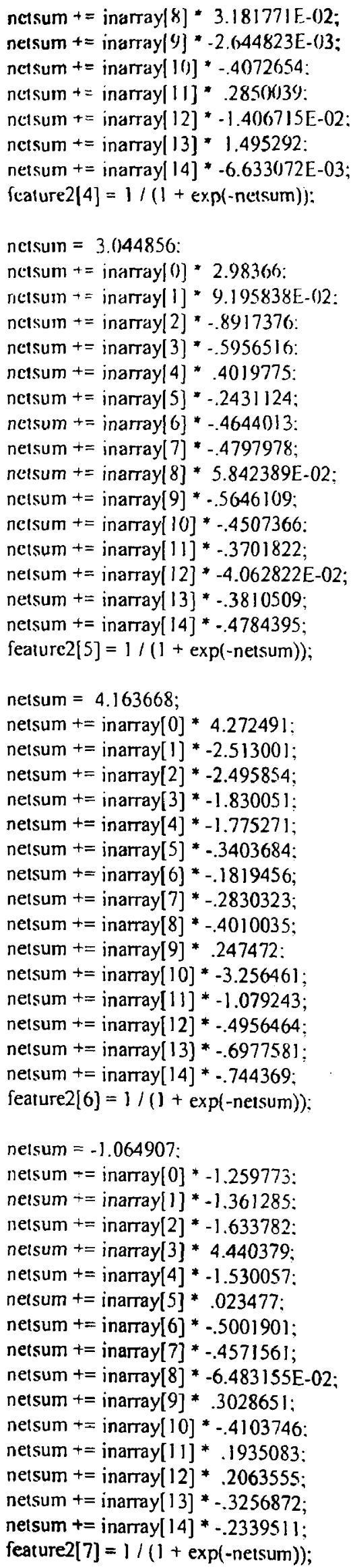




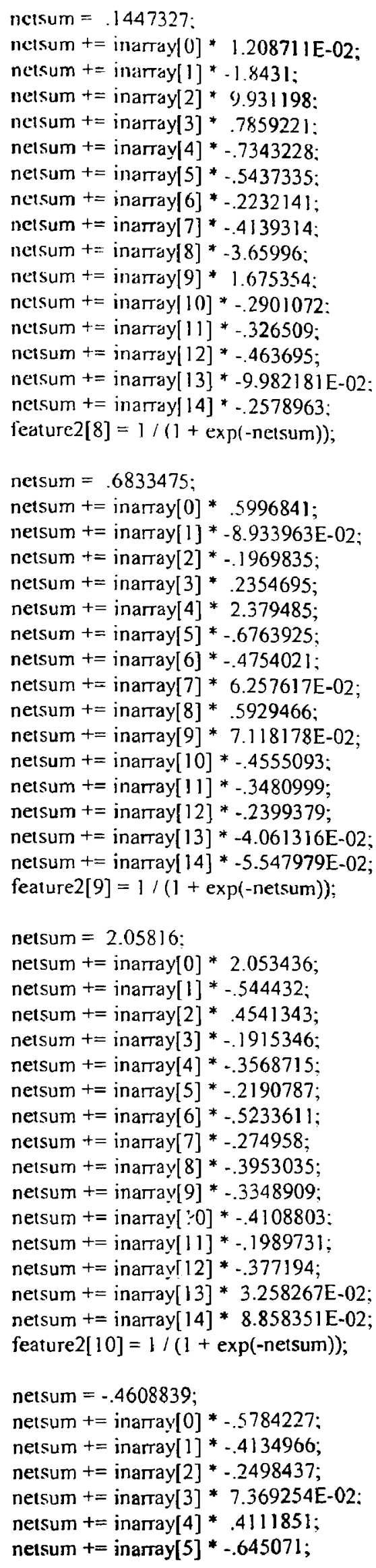




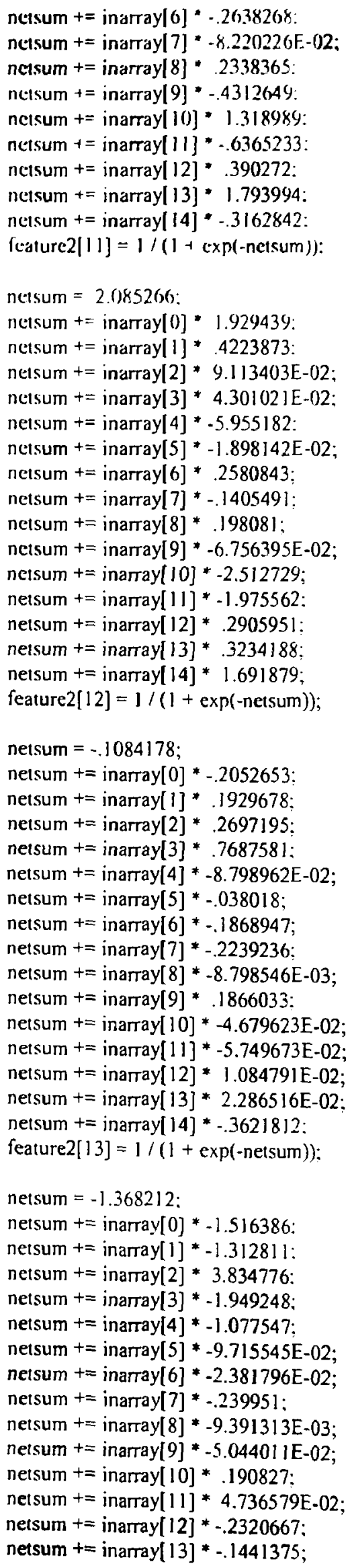




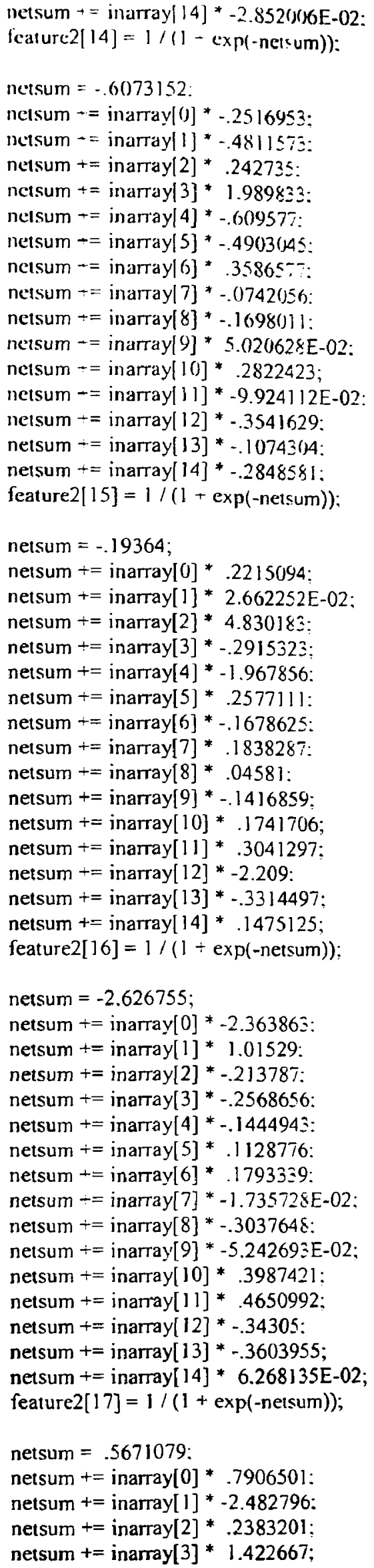




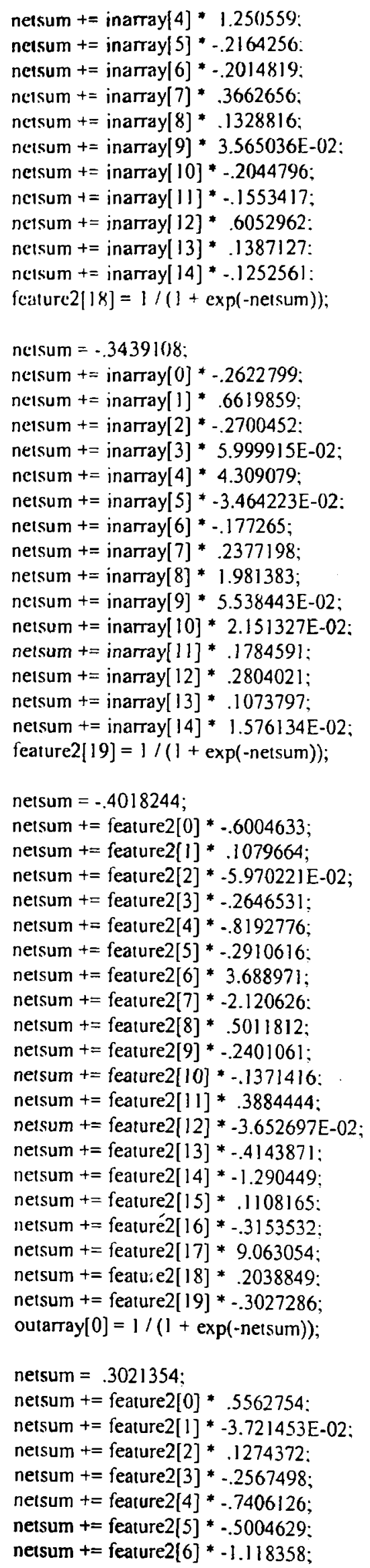




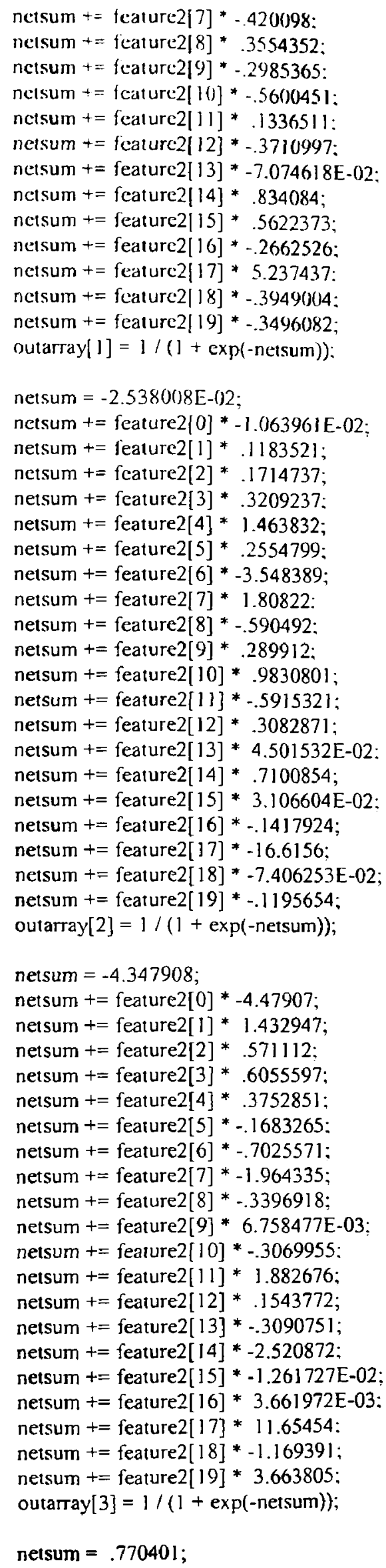




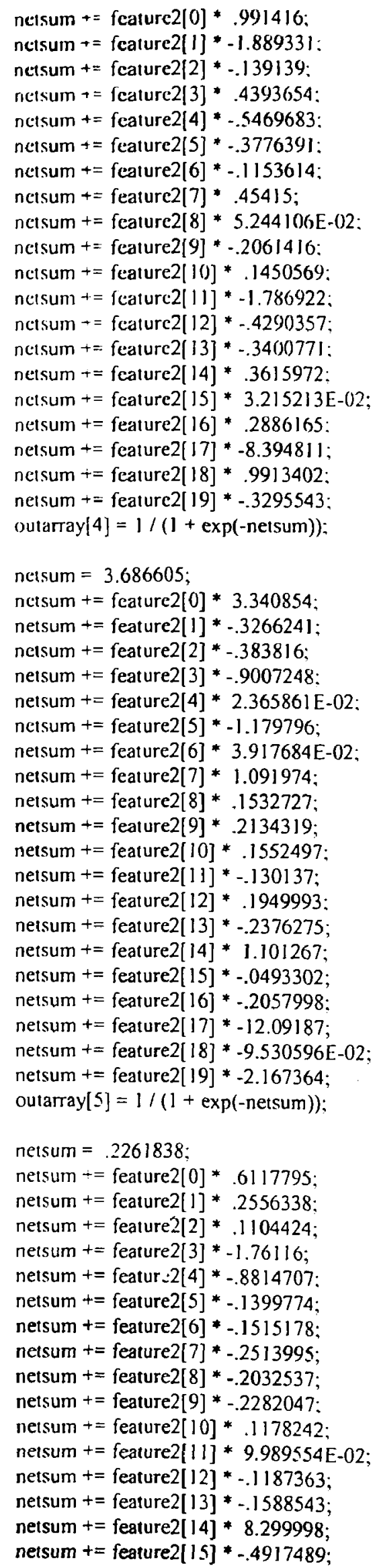




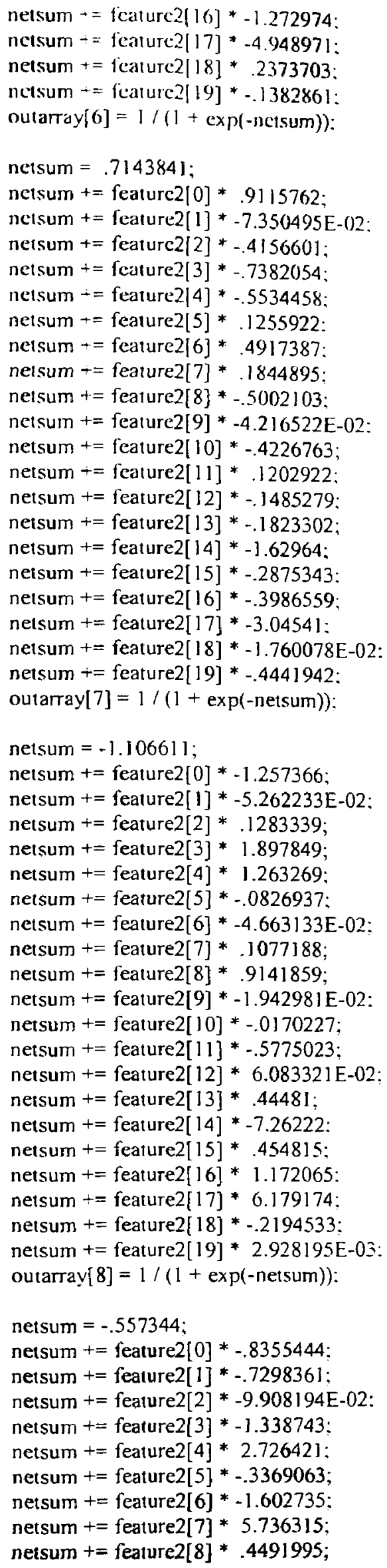




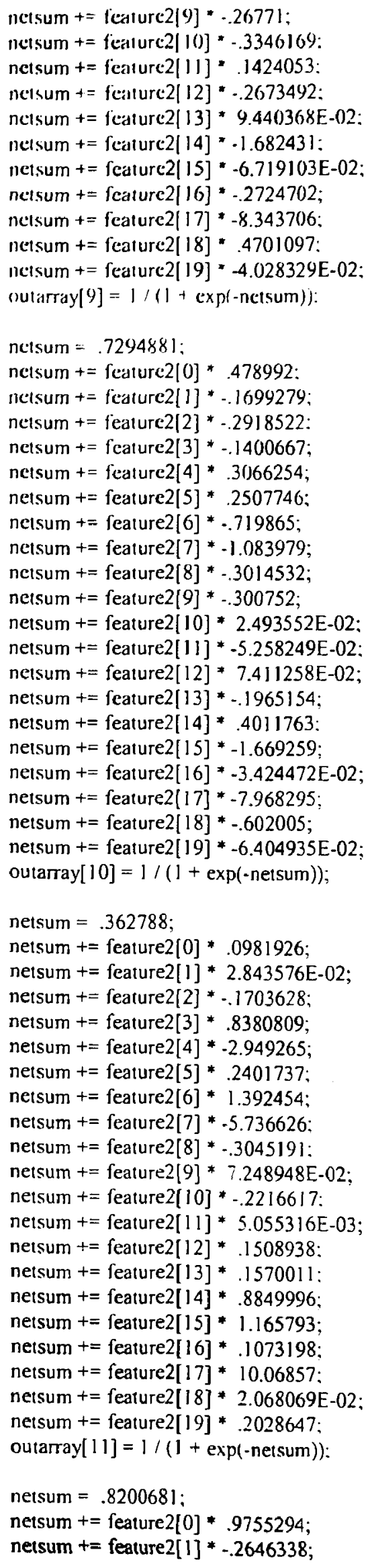




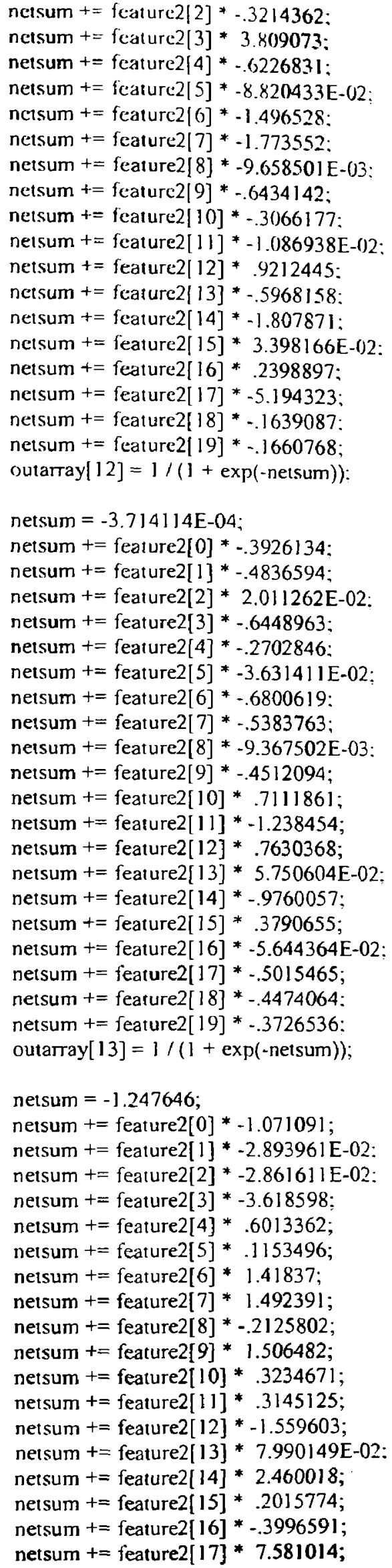




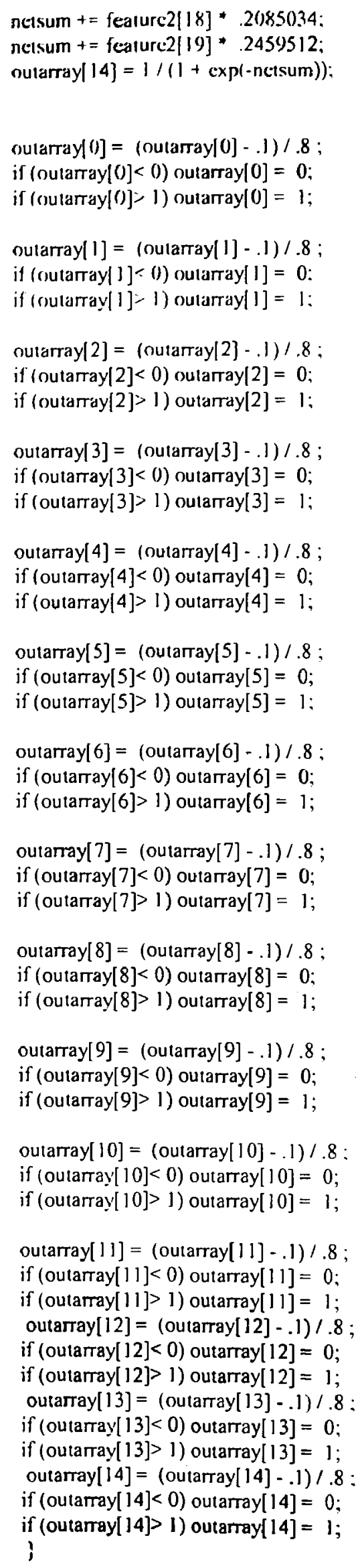


Appendix El:-Comparison of BPNN with makespans from optimal rule combinations for $n=10$ to 100

\begin{tabular}{|c|c|c|c|c|c|c|c|c|c|c|c|}
\hline$n=10$ & Optimal & BPNN & $\mathrm{n}=10$ & Optimal & BPNN & $n=15$ & Optimal & BPNN & $n=15$ & Optimal & BPNN \\
\hline N10M1 & 845 & 845 & N10M26 & 701 & 712 & N15M1 & 1221 & 1272 & N15M26 & 1235 & 1235 \\
\hline N10M2 & 819 & 819 & N10M 27 & 872 & 890 & N15M2 & 1151 & 1151 & N15M27 & 1272 & 1463 \\
\hline N10M3 & 842 & 880 & N10M28 & 604 & 661 & N15M3 & 1127 & 1127 & N15M28 & 1081 & 1111 \\
\hline N10M4 & 762 & 762 & N10M29 & 793 & 793 & N15M4 & 1014 & 1014 & N15M29 & 1125 & 1125 \\
\hline N10M5 & 604 & 604 & N10M30 & 747 & 865 & N15M5 & 1184 & 1184 & N15M30 & 1055 & 1113 \\
\hline N10M6 & 815 & 815 & N10M31 & 834 & 838 & N15M6 & 1238 & 1248 & N15M31 & 1030 & 1051 \\
\hline N10M7 & 723 & 753 & N10M32 & 722 & 781 & N15M7 & 1216 & 1232 & N15M32 & 1064 & 1102 \\
\hline N10M8 & 862 & 862 & N10M33 & 547 & 567 & N15M8 & 1205 & 1286 & N15M 33 & 1040 & 1040 \\
\hline N10M9 & 868 & 899 & N10M34 & 847 & 922 & N15M9 & 1103 & 1103 & N15M34 & 1146 & 1146 \\
\hline N10M10 & 766 & 766 & N10M35 & 734 & 774 & N15M10 & 1078 & 1088 & N15M35 & 1184 & 1190 \\
\hline N10M11 & 800 & 931 & N10M36 & 786 & 825 & N15M11 & 1175 & 1281 & N15M36 & 1185 & 1219 \\
\hline N10M12 & 827 & 828 & N10M37 & 822 & 825 & N15M12 & 1124 & 1124 & N15M37 & 1141 & 1166 \\
\hline N10M13 & 849 & 849 & N10M38 & 785 & 795 & N15M13 & 781 & 781 & N15M38 & 1295 & 1295 \\
\hline N10M14 & 658 & 658 & N10M39 & 763 & 811 & N15M14 & 887 & 926 & N15M39 & 1273 & 1285 \\
\hline N10M15 & 620 & 626 & N10M40 & 540 & 573 & N15M15 & 1141 & 1226 & N15M40 & 1218 & 1218 \\
\hline N10M16 & 801 & 801 & N10M41 & 694 & 733 & N15M16 & 1083 & 1089 & N15M41 & 1140 & 1140 \\
\hline N10M17 & 555 & 555 & N10M42 & 743 & 770 & N15M17 & 1293 & 1293 & N15M42 & 957 & 957 \\
\hline N10M18 & 773 & 319 & N10M43 & 731 & 736 & N15M18 & 1218 & 1286 & N15M43 & 1199 & 1280 \\
\hline N10M19 & 535 & 535 & N10M44 & 690 & 690 & N15M19 & 1089 & 1165 & N15M44 & 1116 & 1170 \\
\hline N10M20 & 872 & 878 & N10M45 & 722 & 722 & N15M20 & 1169 & 1228 & N15M45 & 1159 & 1207 \\
\hline N10M21 & 757 & 757 & N10M46 & 923 & 958 & N15M21 & 974 & 994 & N15M46 & 979 & 979 \\
\hline N10M22 & 592 & 592 & N10M47 & 777 & 820 & N15M22 & 1067 & 1067 & N15M47 & 1299 & 1300 \\
\hline N10M23 & 629 & 641 & N10M48 & 716 & 737 & N15M23 & 844 & 852 & N15M48 & 876 & 918 \\
\hline N10M24 & 811 & 901 & N10M49 & 843 & 862 & N15M24 & 1093 & 1129 & N15M49 & 1155 & 1181 \\
\hline N10M25 & 693 & 693 & N10M50 & 723 & 731 & N15M25 & 1042 & 1122 & N15M50 & 1246 & 1246 \\
\hline
\end{tabular}




\begin{tabular}{|c|c|c|c|c|c|c|c|c|c|c|c|}
\hline$n=20$ & Optimal & BPNN & $n=20$ & Optimal & BPNN & $n=25$ & Optimal & BPNN & $n=25$ & Optimal & BPNN \\
\hline N20M1 & 1532 & 1532 & N20M26 & 1450 & 1450 & $\mathrm{~N} 25 \mathrm{M} 1$ & 1982 & 2028 & N25M26 & 1598 & 1598 \\
\hline N2OM2 & 1426 & 1426 & N20M27 & 1196 & 1196 & N25M2 & 1646 & 1654 & $N 25 M 27$ & 2003 & 2003 \\
\hline N20M3 & 1167 & 1354 & N20M28 & 1492 & 1492 & N25M3 & 1870 & 1870 & N25M 28 & 2015 & 2039 \\
\hline N20M4 & 1566 & 1566 & N20M29 & 1368 & 1370 & N25M4 & 1949 & 1949 & N25M29 & 1538 & 1538 \\
\hline N20M5 & 1476 & 1490 & N20M30 & 1200 & 1307 & N25M5 & 1903 & 1903 & $\mathrm{~N} 25 \mathrm{M} 30$ & 2033 & 2050 \\
\hline N20M6 & 1710 & 1748 & N2OM 31 & 1634 & 1641 & N25M6 & 1680 & 1680 & N25M31 & 2042 & 2042 \\
\hline N20M7 & 1772 & 1781 & N20M32 & 1482 & 1482 & N25M7 & 1838 & 1838 & N25M32 & 1878 & 1878 \\
\hline N20M8 & 1468 & 1468 & N20M33 & 1508 & 1543 & N25M8 & 1978 & 1978 & N25M33 & 2021 & 2021 \\
\hline N20M9 & 1455 & 1455 & N2OM34 & 1740 & 1827 & N25M9 & 2023 & 2023 & N25M34 & 1556 & 1556 \\
\hline N20M10 & 1639 & 1800 & N20M35 & 1441 & 1441 & $\mathrm{~N} 25 \mathrm{M} 10$ & 1727 & 1727 & N25M35 & 1656 & 1656 \\
\hline N20M11 & 1137 & 1196 & N20M36 & 1115 & 1167 & N25M11 & 1817 & 1890 & N25M36 & 1894 & 1895 \\
\hline $\mathrm{N} 20 \mathrm{M} 12$ & 1643 & 1802 & N20M37 & 1386 & 1386 & N25M12 & 1810 & 1810 & N25M37 & 1708 & 1708 \\
\hline $\mathrm{N} 20 \mathrm{M} 13$ & 1476 & 1476 & N20M38 & 1557 & 1557 & N25M13 & 1373 & 1439 & N25M38 & 1501 & 1542 \\
\hline N20M14 & 1194 & 1199 & N20M39 & 1446 & 1448 & N25M14 & 1980 & 1980 & N25M39 & 1846 & 1864 \\
\hline N20M15 & 1351 & 1351 & N2OM40 & 1493 & 1493 & N25M15 & 1693 & 1693 & $\mathrm{~N} 25 \mathrm{M} 40$ & 1824 & 1824 \\
\hline N20M16 & 1617 & 1617 & N2OM41 & 1313 & 1313 & N25M16 & 2019 & 2019 & N25M41 & 1826 & 1881 \\
\hline N20M17 & 1435 & 1474 & N2OM42 & 1476 & 1476 & N25M17 & 2058 & 2058 & $\mathrm{~N} 25 \mathrm{M} 42$ & 1586 & 1586 \\
\hline N20M18 & 1462 & 1462 & N20M43 & 1665 & 1666 & N25M18 & 1889 & 1889 & $\mathrm{~N} 25 \mathrm{M} 43$ & 1817 & 1817 \\
\hline N20M19 & 1496 & 1654 & N2OM44 & 1482 & 1627 & N25M19 & 1788 & 1899 & N25M44 & 1743 & 1743 \\
\hline N2OM20 & 1441 & 1441 & N20M45 & 1336 & 1336 & N25M20 & 1950 & 1950 & N25M45 & 1880 & 1888 \\
\hline N2OM21 & 1327 & 1327 & N20M46 & 1626 & 1697 & N25M21 & 1985 & 1985 & $\mathrm{~N} 25 \mathrm{M} 46$ & 1900 & 1900 \\
\hline N2OM 22 & 1445 & 1474 & N20M47 & 1624 & 1682 & N25M22 & 1864 & 1864 & $\mathrm{~N} 25 \mathrm{M} 47$ & 1587 & 1587 \\
\hline N2OM23 & 1270 & 1270 & N20M48 & 1592 & 1592 & N25M23 & 1611 & 1611 & N25M48 & 1877 & 1877 \\
\hline N20M24 & 1059 & 1059 & N20M49 & 1490 & 1490 & N25M24 & 1769 & 1769 & N25M49 & 1695 & 1695 \\
\hline N20M25 & 1288 & 1304 & N20M50 & 1534 & 1534 & N25M25 & 1709 & 1776 & N25M50 & 1612 & 1612 \\
\hline
\end{tabular}




\begin{tabular}{|c|c|c|c|c|c|c|c|c|c|c|c|}
\hline$n=30$ & Optimal & BPNN & $n=30$ & Optimal & BPNN & $n=35$ & Optimal & BPNN & $n=35$ & Optimal & BPNN \\
\hline N30M1 & 2270 & 2332 & N30M26 & 2254 & 2254 & N35M1 & 2787 & 2793 & N35M26 & 2741 & 2741 \\
\hline N30M2 & 2097 & 2097 & N30M27 & 2325 & 2325 & N35M2 & 2561 & 2561 & N35M27 & 2712 & 2712 \\
\hline N30M3 & 1788 & 1788 & N30M28 & 2282 & 2282 & N35M3 & 2745 & 2789 & N35M28 & 2595 & 2595 \\
\hline N30M4 & 1971 & 1971 & N30M29 & 2051 & 2057 & N35M4 & 2361 & 2361 & N35M29 & 2020 & 2020 \\
\hline N30M5 & 2280 & 2280 & N30M30 & 2136 & 2136 & N35M5 & 2727 & 2727 & N35M30 & 2808 & 2808 \\
\hline N30M6 & 2229 & 2385 & N30M31 & 1981 & 2075 & N35M6 & 2707 & 2743 & N35M31 & 2705 & 2705 \\
\hline N30M7 & 2016 & 2016 & N30M32 & 1973 & 1973 & N35M7 & 2602 & 2772 & N35M32 & 2218 & 2218 \\
\hline N30M8 & 2426 & 2675 & N30M33 & 2047 & 2047 & N35M8 & 2768 & 2768 & N35M33 & 2062 & 2174 \\
\hline N30M9 & 2219 & 2219 & N30M34 & 2238 & 2238 & N35M9 & 2666 & 3099 & N35M34 & 2643 & 2685 \\
\hline N30M10 & 1778 & 1790 & N30M35 & 2046 & 2050 & N35M10 & 2622 & 2622 & N35M35 & 2544 & 2544 \\
\hline N30M11 & 2113 & 2166 & N30M36 & 2456 & 2456 & N35M11 & 2749 & 2749 & N35M36 & 2654 & 2654 \\
\hline N30M 12 & 2149 & 2149 & N30M37 & 2271 & 2271 & N35M12 & 2334 & 2355 & N35M37 & 2066 & 2166 \\
\hline N30M13 & 2171 & 2171 & N30M38 & 2181 & 2319 & N35M13 & 2716 & 2716 & N35M38 & 2623 & 2623 \\
\hline N30M14 & 2151 & 2151 & N30M39 & 2238 & 2299 & N35M14 & 2743 & 2743 & N35M39 & 2569 & 2569 \\
\hline N30M15 & 2053 & 2053 & N30M40 & 2172 & 2309 & N35M15 & 2482 & 2482 & N35M40 & 2241 & 2241 \\
\hline N30M16 & 2009 & 2045 & N30M41 & 2377 & 2377 & N35M16 & 2459 & 2459 & N35M41 & 2636 & 2636 \\
\hline N30M17 & 2104 & 2130 & N30M42 & 2076 & 2091 & N35M17 & 2654 & 2654 & N35M42 & 2397 & 2502 \\
\hline N30M18 & 2367 & 2367 & N3OM43 & 2278 & 2391 & N35M18 & 2308 & 2308 & N35M43 & 2123 & 2129 \\
\hline N30M19 & 2227 & 2356 & N30M44 & 2005 & 2005 & N35M 19 & 2585 & 2585 & N35M44 & 2592 & 2749 \\
\hline N30M20 & 2251 & 2273 & N30M45 & 2347 & 2628 & N35M20 & 2670 & 2670 & N35M45 & 2496 & 2496 \\
\hline N30M21 & 2049 & 2049 & N30M46 & 2132 & 2192 & N35M21 & 2308 & 2308 & N35M46 & 2604 & 2604 \\
\hline N30M22 & 2359 & 2359 & N30M47 & 2078 & 2078 & N35M22 & 2254 & 2254 & N35M47 & 2192 & 2192 \\
\hline N30M23 & 2300 & 2300 & N30M48 & 2302 & 2391 & N35M23 & 2555 & 2555 & N35M48 & 2255 & 2255 \\
\hline N30M24 & 2444 & 2444 & N30M49 & 2150 & 2252 & N35M24 & 2725 & 2725 & N35M49 & 2613 & 2613 \\
\hline N30M25 & 1650 & 1650 & N30M50 & 1964 & 1964 & N35M25 & 2405 & 2405 & N35M50 & 2705 & 2705 \\
\hline
\end{tabular}




\begin{tabular}{|c|c|c|c|c|c|c|c|c|c|c|c|}
\hline$n=40$ & Optimal & BPNN & $n=40$ & Optimal & BPNN & $n=45$ & Optimal & BPNN & $n=45$ & Optimal & BPNN \\
\hline N40M1 & 2797 & 2797 & N40M26 & 3033 & 3033 & N45M1 & 3674 & 4104 & N45M26 & 3274 & 3455 \\
\hline N40M2 & 2621 & 2621 & $\mathrm{~N} 40 \mathrm{M} 27$ & 3072 & 3072 & N45M2 & 3348 & 3374 & $\mathrm{~N} 45 \mathrm{M} 27$ & 3301 & 3301 \\
\hline N40M3 & 3079 & 3079 & N40M28 & 3252 & 3456 & $\mathrm{~N} 45 \mathrm{M} 3$ & 3298 & 3298 & N45M28 & 2902 & 2902 \\
\hline N40M4 & 3040 & 3040 & N40M 29 & 2882 & 2882 & N45M4 & 2883 & 2883 & $\mathrm{~N} 45 \mathrm{M} 29$ & 3531 & 3531 \\
\hline N40M5 & 2674 & 2752 & N40M30 & 2999 & 3005 & N45M5 & 3251 & 3251 & N45M30 & 3297 & 3297 \\
\hline N40M6 & 2852 & 2862 & N40M31 & 3120 & 3120 & N45M6 & 3456 & 3456 & N45M31 & 2846 & 2931 \\
\hline N40M7 & 2111 & 2111 & N40M32 & 2664 & 2681 & N45M7 & 2899 & 2899 & N45M32 & 3599 & 3637 \\
\hline N40M8 & 2153 & 2183 & N40M33 & 3065 & 3065 & N45M8 & 3446 & 3446 & N45M33 & 3394 & 3400 \\
\hline N40M9 & 2670 & 2670 & N40M34 & 2901 & 2906 & N45M9 & 3206 & 3206 & N45M34 & 3047 & 3047 \\
\hline N40M10 & 2364 & 2364 & N40M35 & 2981 & 2981 & N45M10 & 2484 & 2484 & N45M 35 & 3763 & 3922 \\
\hline N40M11 & 2952 & 2952 & N40M36 & 2789 & 2789 & N45M11 & 3034 & 3034 & N45M36 & 3135 & 3158 \\
\hline N40M12 & 2314 & 2314 & N40M37 & 3080 & 3138 & N45M12 & 2532 & 2638 & N45M37 & 2829 & 2829 \\
\hline N40M13 & 2445 & 2445 & N40M38 & 2511 & 2511 & $N 45 M 13$ & 2953 & 2953 & N45M38 & 3382 & 3382 \\
\hline N40M14 & 2693 & 2693 & N40M39 & 2722 & 2722 & N45M14 & 3256 & 3310 & N45M39 & 3228 & 3228 \\
\hline N40M15 & 2078 & 2241 & N40M40 & 1979 & 1979 & N45M15 & 3727 & 3792 & N45M40 & 3676 & 3676 \\
\hline N40M16 & 3062 & 3062 & N40M41 & 2321 & 2321 & N45M16 & 3399 & 3415 & N45M41 & 2927 & 2927 \\
\hline N40M17 & 2636 & 2636 & N40M42 & 3003 & 3003 & N45M17 & 3060 & 3060 & N45M42 & 2894 & 2894 \\
\hline N40M18 & 2706 & 2727 & N40M43 & 3107 & 3123 & N45M18 & 2749 & 2749 & $\mathrm{~N} 45 \mathrm{M} 43$ & 3280 & 3352 \\
\hline N40M19 & 3112 & 3112 & N40M44 & 3101 & 3101 & N45M19 & 3425 & 3425 & N45M44 & 2917 & 2917 \\
\hline N40M2O & 2843 & 2843 & N40M45 & 2944 & 2944 & N45M20 & 3060 & 3060 & N45M45 & 3400 & 3400 \\
\hline N40M21 & 2900 & 2900 & N40M46 & 3264 & 3450 & N45M21 & 3456 & 3456 & N45M46 & 2771 & 2781 \\
\hline N4OM 22 & 2232 & 2232 & N40M47 & 2953 & 2976 & N45M22 & 3268 & 3268 & N45M47 & 3410 & 3845 \\
\hline $\mathrm{N} 40 \mathrm{M} 23$ & 3059 & 3059 & N40M48 & 3254 & 3254 & N45M23 & 3580 & 4289 & N45M48 & 3374 & 3374 \\
\hline N40M24 & 2710 & 2713 & N40M49 & 2388 & 2566 & N45M24 & 3469 & 3469 & N45M49 & 3468 & 3481 \\
\hline N40M25 & 2512 & 2512 & N40M50 & 2829 & 2829 & N45M25 & 2504 & 2553 & N45M50 & 3283 & 3283 \\
\hline
\end{tabular}




\begin{tabular}{|c|c|c|c|c|c|c|c|c|c|c|c|}
\hline$n=55$ & Optimal & BPNN & $n=55$ & Optimal & BPNN & $n=60$ & Optimal & BPNN & $n=60$ & Optimal & BPNN \\
\hline N55M1 & 4111 & 4111 & N605M26 & 3706 & 3706 & N60M1 & 4001 & 4001 & N60M26 & 4500 & 4500 \\
\hline N55M2 & 4382 & 4407 & N55M27 & 4209 & 4209 & N60M2 & 4556 & 4556 & N60M27 & 4218 & 4218 \\
\hline N55M3 & 3786 & 3786 & N55M28 & 3855 & 3855 & N60M3 & 4562 & 4562 & N60M28 & 3348 & 3348 \\
\hline N55M4 & 3898 & 4090 & N55M29 & 4124 & 4124 & N60M4 & 4197 & 4197 & N60M29 & 4755 & 4811 \\
\hline N55M5 & 4084 & 4084 & N55M30 & 4135 & 4138 & N60M5 & 3927 & 3927 & N60M30 & 4669 & 4669 \\
\hline N55M6 & 3742 & 4037 & N55M31 & 4088 & 4173 & N60M6 & 4801 & 4801 & N60M31 & 4134 & 4134 \\
\hline N55M7 & 3956 & 3970 & N55M32 & 3734 & 3753 & N60M7 & 3915 & 3915 & N60M32 & 4556 & 4556 \\
\hline N55M8 & 3531 & 3531 & N55M33 & 4282 & 4282 & N60M8 & 4523 & 4523 & N60M33 & 4406 & 4420 \\
\hline N55M9 & 4015 & 4015 & N55M34 & 4303 & 4303 & N60M9 & 3733 & 3733 & N60M34 & 3492 & 3495 \\
\hline N55M 10 & 3625 & 3625 & N55M35 & 3761 & 3761 & N60M 10 & 4697 & 4697 & N60M35 & 4583 & 4583 \\
\hline N55M11 & 4113 & 4113 & N55M36 & 4225 & 4225 & N60M11 & 3876 & 3876 & N60M36 & 3821 & 3821 \\
\hline N55M12 & 3964 & 3981 & N55M37 & 3919 & 3919 & N60M 12 & 4546 & 4546 & N60M37 & 4292 & 4292 \\
\hline N55M13 & 4413 & 4531 & N55M38 & 3914 & 3914 & $\mathrm{~N} 60 \mathrm{M} 13$ & 4148 & 4148 & N60M38 & 4451 & 4451 \\
\hline N55M14 & 3586 & 3586 & N55M39 & 4060 & 4060 & N60M 14 & 4585 & 4585 & N60M39 & 4260 & 4260 \\
\hline N55M15 & 4321 & 4698 & N55M40 & 3487 & 3487 & N60M 15 & 3186 & 3186 & $\mathrm{~N} 60 \mathrm{M} 40$ & 4659 & 4659 \\
\hline N55M16 & 3771 & 3906 & N55M41 & 3820 & 3820 & N60M16 & 4599 & 4599 & N60M41 & 4558 & 4627 \\
\hline N55M17 & 3950 & 4060 & N55M42 & 3984 & 3984 & N60M17 & 4225 & 4225 & N60M42 & 4243 & 4243 \\
\hline N55M18 & 4205 & 4205 & N55M43 & 3955 & 3955 & N60M18 & 4443 & 4490 & N60M43 & 4535 & 4535 \\
\hline N55M19 & 4281 & 4281 & N55M44 & 4325 & 4325 & N60M19 & 4135 & 4135 & N60M44 & 4547 & 4773 \\
\hline N55M20 & 3670 & 3670 & N55M45 & 3961 & 3965 & N60M20 & 4060 & 4060 & N60M45 & 4660 & 4660 \\
\hline N55M21 & 3920 & 3920 & N55M46 & 4256 & 4256 & N60M 21 & 4770 & 4770 & N60M46 & 3935 & 3935 \\
\hline N55M22 & 3461 & 3461 & N55M47 & 4011 & 4011 & N60M22 & 4460 & 4460 & N60M47 & 4657 & 4657 \\
\hline N55M23 & 4282 & 4282 & $\mathrm{~N} 55 \mathrm{M} 48$ & 4220 & 4220 & $\mathrm{~N} 60 \mathrm{M} 23$ & 3591 & 3703 & N60M48 & 4552 & 4552 \\
\hline N55M24 & 3600 & 3600 & N55M49 & 3768 & 3768 & N60M24 & 4304 & 4304 & N60M49 & 4274 & 4441 \\
\hline N55M25 & 4451 & 4760 & N55M50 & 4106 & 4106 & N60M 25 & 4574 & 4574 & N60M50 & 4379 & 4379 \\
\hline
\end{tabular}




\begin{tabular}{|c|c|c|c|c|c|c|c|c|c|c|c|}
\hline$n=75$ & Optimal & BPNN & $n=75$ & Optimal & BPNN & $n=85$ & Optimal & BPNN & $n=85$ & Optimal & BPNN \\
\hline N75M1 & 5568 & 5568 & N75M26 & 5264 & 5264 & N85M1 & 5950 & 5950 & N85M26 & 6316 & 6316 \\
\hline $\mathrm{N} 75 \mathrm{M} 2$ & 5536 & 5969 & N75M27 & 5836 & 5836 & N85M2 & 5819 & 5819 & N85M27 & 6457 & 6457 \\
\hline N75M3 & 5246 & 5246 & N75M28 & 4985 & 4985 & N85M3 & 6523 & 6523 & N85M28 & 5559 & 5559 \\
\hline N75M4 & 4936 & 4936 & N75M29 & 5962 & 5962 & N85M4 & 6274 & 6274 & N85M29 & 5553 & 5553 \\
\hline N75M5 & 5544 & 5907 & N75M30 & 3991 & 3991 & N85M5 & 5745 & 5745 & N85M30 & 6054 & 6054 \\
\hline N75M6 & 5820 & 6161 & N75M31 & 5220 & 5220 & N85M6 & 6642 & 6642 & N85M31 & 5892 & 5892 \\
\hline N75M7 & 4026 & 4055 & N75M32 & 5792 & 5792 & N85M7 & 5748 & 5806 & N85M32 & 6046 & 6046 \\
\hline N75M8 & 5602 & 5602 & N75M33 & 5161 & 5161 & N85M8 & 6781 & 6962 & N85M33 & 5660 & 5660 \\
\hline N75M9 & 5890 & 5890 & N75M34 & 5413 & 5436 & N85M9 & 5835 & 5835 & N85M34 & 5871 & 5871 \\
\hline N75M10 & 5433 & 5433 & N75M35 & 5526 & 5526 & N85M 10 & 6740 & 6740 & N85M35 & 6243 & 6243 \\
\hline N75M11 & 5794 & 5794 & N75M36 & 5372 & 5372 & N85M11 & 5526 & 5526 & N85M36 & 6169 & 6169 \\
\hline$N 75 M 12$ & 5503 & 5503 & N75M37 & 5155 & 5157 & N85M12 & 6446 & 6446 & N85M37 & 6412 & 6504 \\
\hline N75M13 & 5153 & 5153 & N75M38 & 4822 & 5010 & N85M13 & 6242 & 6242 & N85M38 & 6272 & 6272 \\
\hline N75M14 & 5244 & 5244 & N75M39 & 5823 & 5823 & N85M14 & 5973 & 5973 & N85M39 & 6263 & 6263 \\
\hline N75M15 & 4750 & 5270 & N75M40 & 5714 & 5714 & N85M15 & 6231 & 6231 & N85M40 & 4396 & 4464 \\
\hline $\mathrm{N} 75 \mathrm{M} 16$ & 5737 & 5737 & N75M41 & 5215 & 5215 & N85M16 & 6485 & 6485 & N85M41 & 6259 & 6259 \\
\hline $\mathrm{N} 75 \mathrm{M} 17$ & 5947 & 5974 & N75M42 & 5706 & 5751 & N85M17 & 5512 & 5512 & N85M42 & 6206 & 6206 \\
\hline N75M18 & 5712 & 5712 & N75M43 & 5154 & 5154 & N85M18 & 6100 & 6100 & N85M43 & 6564 & 6564 \\
\hline N75M19 & 5479 & 5479 & N75M44 & 5815 & 6093 & N85M 19 & 6345 & 6345 & N85M44 & 6727 & 6741 \\
\hline $\mathrm{N} 75 \mathrm{M} 20$ & 5364 & 5881 & N75M45 & 4914 & 4914 & N85M20 & 6447 & 6447 & N85M45 & 5081 & 5081 \\
\hline N75M21 & 5870 & 5881 & N75M46 & 4343 & 4343 & N85M 21 & 5815 & 5815 & N85M46 & 5842 & 5842 \\
\hline N75M22 & 4265 & $\grave{4} 265$ & N75M47 & 5651 & 5651 & N85M22 & 6603 & 6603 & N85M47 & 6706 & 6706 \\
\hline N75M23 & 5619 & 5650 & N75M48 & 5427 & 5427 & N85M23 & 6513 & 6513 & N85M48 & 5983 & 5983 \\
\hline N75M24 & 5692 & 5692 & N75M49 & 5779 & 5779 & N85M24 & 5571 & 6108 & N85M49 & 4801 & 4801 \\
\hline N75M25 & 5470 & 5470 & N75M50 & 5578 & 5578 & N85M25 & 6056 & 6056 & N85M50 & 6138 & 6138 \\
\hline
\end{tabular}




\begin{tabular}{|c|c|c|c|c|c|}
\hline $\mathrm{n}=100$ & Optimal & BPNN & $\mathrm{n}=100$ & Optimal & BPNN \\
\hline N100M1 & 5379 & 5849 & N100M26 & 7279 & 7279 \\
N100M2 & 7571 & 7571 & N100M27 & 7116 & 7116 \\
N100M3 & 7187 & 7324 & N100M28 & 6991 & 6991 \\
N100M4 & 6445 & 6445 & N100M29 & 5654 & 5654 \\
N100M5 & 7727 & 7727 & N100M30 & 7737 & 7737 \\
N100M6 & 7382 & 7408 & N100M31 & 6685 & 6685 \\
N100M7 & 7605 & 7605 & N100M32 & 7244 & 7244 \\
N100M8 & 7616 & 7616 & N100M33 & 7410 & 7411 \\
N100M9 & 6866 & 6866 & N100M34 & 7427 & 7427 \\
N100M10 & 7940 & 7952 & N100M35 & 6858 & 7113 \\
N100M11 & 7810 & 7810 & N100M36 & 7497 & 7497 \\
N100M12 & 6931 & 6931 & N100M37 & 7739 & 7739 \\
N100M13 & 7705 & 7705 & N100M38 & 7571 & 7571 \\
N100M14 & 7183 & 7183 & N100M39 & 7709 & 7709 \\
N100M15 & 6204 & 6204 & N100M40 & 5503 & 5516 \\
N100M16 & 6479 & 6479 & N100M41 & 7707 & 7707 \\
N100M17 & 5926 & 5926 & N100M42 & 7178 & 7178 \\
N100M18 & 6189 & 6189 & N100M43 & 6310 & 6491 \\
N100M19 & 7839 & 7839 & N100M44 & 7210 & 7210 \\
N100M20 & 7360 & 7388 & N100M45 & 5799 & 5799 \\
N100M21 & 6124 & 6124 & N100M46 & 7897 & 7901 \\
N100M22 & 6901 & 6901 & N100M47 & 7037 & 7037 \\
N100M23 & 6862 & 6862 & N100M48 & 7212 & 7212 \\
N100M24 & 7667 & 7667 & N100M49 & 7526 & 7526 \\
N100M25 & 6276 & 6276 & N100M50 & 7244 & 7244 \\
\hline
\end{tabular}


Appendix E2: -Comparison of BPNN with Mean Flowtimes for optimal rule combinations for $n=10$ to 100

\begin{tabular}{|c|c|c|c|c|c|c|c|c|c|c|c|}
\hline$n=10$ & Optimal & BPNN & $n=10$ & Optimal & BPNN & $n=15$ & Optimal & BPNN & $n=15$ & Optimal & BPNN \\
\hline N10F1 & 560.5 & 563.6 & N10F26 & 471.2 & 475.3 & N15F 1 & 782.69 & 794.67 & N15F26 & 791.67 & 807.73 \\
\hline N10F2 & 518 & 554.6 & N10F27 & 671.3 & 708.3 & N15F2 & 678.67 & 679.27 & $N 15 F 27$ & 798.8 & 814.33 \\
\hline N10F3 & 559.2 & 569.1 & N10F28 & 445.7 & 487.7 & N15F3 & 754.87 & 781.67 & $N 15 F 28$ & 670.53 & 689.4 \\
\hline N10F4 & 539.7 & 588.6 & N10F 29 & 502.6 & 512.2 & N15F4 & 623.13 & 631.2 & N15F29 & 659.33 & 659.33 \\
\hline N10F5 & 400.8 & 409.4 & N10F 30 & 533.3 & 544.9 & N15F5 & 750.53 & 756.53 & N15F 30 & 704.13 & 748.67 \\
\hline N10F6 & 599.7 & 599.7 & N10F31 & 585.1 & 595.8 & N15F6 & 770.13 & 776.67 & N15F31 & 649.33 & 649.33 \\
\hline N10F 7 & 510.7 & 519.8 & N10F32 & 525.6 & 555.7 & N15F7 & 774.13 & 783.73 & N15F32 & 737.73 & 737.73 \\
\hline N10F8 & 621.2 & 633 & N10F33 & 371.2 & 386.5 & N15F8 & 816.87 & 823.33 & N15F33 & 687.6 & 707.27 \\
\hline N10F9 & 605.9 & 605.9 & N10F34 & 590.2 & 619.6 & N15F9 & 674.67 & 702.93 & N15F34 & 770.67 & 757.73 \\
\hline N10F 10 & 534.6 & 534.8 & N10F35 & 514.7 & 544.1 & N15F 10 & 701.33 & 707 & N15F35 & 672 & 798.93 \\
\hline N10F11 & 569.5 & 579.9 & N10F36 & 592.3 & 612.1 & N15F11 & 709.2 & 732.4 & N15F36 & 586.53 & 758.2 \\
\hline N1OF 12 & 535.4 & 562.7 & N10F37 & 539.8 & 550.8 & $\mathrm{~N} 15 \mathrm{~F} 12$ & 656.47 & 656.47 & N15F37 & 600.8 & 732.07 \\
\hline $\mathrm{N} 10 \mathrm{~F} 13$ & 583.1 & 591.5 & N10F38 & 560.4 & 591 & $N 15 F 13$ & 492.13 & 515.2 & N15F38 & 612.4 & 799.6 \\
\hline N10F 14 & 475.1 & 479.9 & N10F39 & 523.1 & 527.5 & N15F 14 & 536.4 & 538.53 & N15F39 & 819 & 785.93 \\
\hline N10F 15 & 464.7 & 469.2 & N10F40 & 391.1 & 391.1 & N15F 15 & 713.53 & 720.6 & N15F40 & 651.13 & 830.67 \\
\hline N1OF16 & 536.7 & 536.7 & N10F41 & 453.5 & 459 & N15F16 & 687.07 & 698.87 & N15F41 & 707.33 & 713.67 \\
\hline N10F17 & 404.7 & 419.6 & $\mathrm{~N} 10 \mathrm{~F} 42$ & 546.3 & 555.5 & N15F17 & 798.13 & 834.07 & N15F42 & 799.13 & 637.67 \\
\hline N10F18 & 554.6 & 573.4 & $N 10 F 43$ & 531.4 & 531.4 & N15F18 & 741.2 & 744.2 & N15F43 & 594.2 & 816.67 \\
\hline N10F19 & 394.1 & 406.2 & N10F44 & 461.2 & 503.2 & N15F19 & 688.93 & 720.13 & N15F44 & 721.6 & 669.33 \\
\hline N10F20 & 633 & 648.8 & N10F45 & 508.9 & 522.5 & N15F20 & 762.47 & 800.6 & N15F45 & 632.67 & 775.87 \\
\hline N1OF 21 & 508.2 & 583 & N10F 46 & 641.7 & 658.9 & N15F21 & 625.87 & 646.47 & N15F46 & 716.13 & 646.93 \\
\hline N10F22 & 403.4 & 417.9 & N10F 47 & 560.6 & 560.6 & N15F22 & 659.73 & 684.33 & N15F47 & 726.47 & 815.2 \\
\hline $\mathrm{N} 10 \mathrm{~F} 23$ & 387.1 & 397.7 & N10F48 & 524 & 541.5 & N15F23 & 564.6 & 568.93 & N15F48 & 509.13 & 583 \\
\hline N10F24 & 558 & 590.8 & N10F49 & 582 & 609.2 & N15F24 & 721.33 & 744.13 & N15F49 & 558.07 & 731.4 \\
\hline N10F 25 & 500.4 & 511.6 & N10F50 & 489.6 & 497.4 & N15F25 & 633.87 & 656.73 & N15F50 & 807.93 & 826.6 \\
\hline
\end{tabular}




\begin{tabular}{|c|c|c|c|c|c|c|c|c|c|c|c|}
\hline$n=20$ & Optimal & BPNN & $n=20$ & Optimal & BPNN & $n=25$ & Optimal & BPNN & $\mathrm{n}=25$ & Optimal & BPNN \\
\hline N2OF1 & 933.35 & 945.95 & N2OF26 & 867.25 & 876.15 & $\mathrm{~N} 25 \mathrm{~F} 1$ & 1186 & 1187.4 & N25F26 & 963.16 & 966.4 \\
\hline N20F2 & 896.5 & 897.8 & N20F 27 & 703.8 & 703.8 & N25F2 & 961.2 & 974.48 & N25F 27 & 1152.88 & 1168.36 \\
\hline N20F3 & 705.75 & 738.45 & N20F 28 & 923.1 & 923.1 & N25F3 & 1025.92 & 1026.92 & N25F 28 & 1112 & 1120.12 \\
\hline N2OF4 & 864.75 & 867.45 & N20F29 & 826.55 & 846.6 & N25F4 & 1142.08 & 1210.16 & N25F29 & 824.96 & 827.2 \\
\hline N20F5 & 827.85 & 852.7 & N20F30 & 777.1 & 803.95 & N25F5 & 1107.44 & 1125.52 & $\mathrm{~N} 25 \mathrm{~F} 30$ & 1185.56 & 1209.96 \\
\hline N20F6 & 969.2 & 1050.05 & N20F31 & 952.35 & 955.3 & N25F6 & 972.08 & 972.08 & $\mathrm{~N} 25 \mathrm{~F} 31$ & 1083.92 & 1098.64 \\
\hline N20F7 & 1052.35 & 1052.35 & N20F32 & 902.9 & 950.9 & $\mathrm{~N} 25 \mathrm{~F} 7$ & 1101.2 & 1106.04 & N25F32 & 1080.92 & 1086.4 \\
\hline N20F8 & 824.4 & 830.15 & N2OF33 & 862 & 866.3 & N25F 8 & 1082.12 & 1092.48 & N25F 33 & 1259.16 & 1297.28 \\
\hline N20F9 & 848.05 & 923.05 & N20F34 & 1019.8 & 1053.4 & N25F9 & 1148.68 & 1160.12 & N25F34 & 866.6 & 870 \\
\hline N2OF 10 & 1013.83 & 1051.9 & N20F35 & 823.55 & 823.55 & N25F 10 & 1023.4 & 1038.76 & N25F35 & 958.72 & 970.8 \\
\hline N2OF 11 & 710.45 & 713.55 & N20F36 & 660.6 & 665.75 & N25F11 & 1025.56 & 1025.56 & N25F 36 & 989.76 & 1030 \\
\hline $\mathrm{N} 2 \mathrm{OF} 12$ & 1000.95 & 1018.25 & N20F37 & 780.35 & 787.9 & $\mathrm{~N} 25 \mathrm{~F} 12$ & 979.28 & 992.44 & N25F 37 & 996.12 & 1002.04 \\
\hline N20F 13 & 881.3 & 932.2 & N20F38 & 973.85 & 983.55 & N25F 13 & 820.08 & 837.04 & N25F38 & 846.08 & 880.6 \\
\hline N2OF 14 & 747.4 & 747.4 & N20F39 & 819.2 & 821.55 & N25F 14 & 1191 & 1226.6 & N25F39 & 1018.76 & 1028.96 \\
\hline N20F 15 & 766.55 & 767.15 & $\mathrm{~N} 20 \mathrm{~F} 40$ & 870.7 & 882.85 & N25F15 & 967.72 & 967.72 & $\mathrm{~N} 25 \mathrm{~F} 40$ & 1000.52 & 1000.68 \\
\hline N20F 16 & 966.95 & 976.65 & N20F41 & 756.4 & 767.15 & $N 25 F 16$ & 1145.16 & 1147.64 & N25F 41 & 1041.16 & 1056.24 \\
\hline $\mathrm{N} 20 \mathrm{~F} 17$ & 870.6 & 870.6 & N20F42 & 858.35 & 858.35 & N25F 17 & 1144.44 & 1163.16 & N25F42 & 908.8 & 927.16 \\
\hline N20F 18 & 857.15 & 859.45 & $\mathrm{~N} 20 \mathrm{~F} 43$ & 1014.4 & 1051.8 & N25F 18 & 1029.08 & 1075.64 & $N 25 F 43$ & 946.68 & 999.04 \\
\hline N20F19 & 917.7 & 917.7 & N20F44 & 908.6 & 910.9 & $\mathrm{~N} 25 \mathrm{~F} 19$ & 1031.52 & 1034.12 & $\mathrm{~N} 25 \mathrm{~F} 44$ & 1036.6 & 1055.48 \\
\hline N20F20 & 859.3 & 873.9 & N20F45 & 787.4 & 808.5 & $\mathrm{~N} 25 \mathrm{~F} 20$ & 1096.28 & 1096.28 & N25F 45 & 1056.16 & 1109.72 \\
\hline N20F21 & 746.75 & 753.7 & N20F46 & 1034.45 & 1064.1 & N25F21 & 1094 & 1101 & $\mathrm{~N} 25 \mathrm{~F} 46$ & 1090 & 1135.52 \\
\hline N20F22 & 907.95 & 932.35 & $\mathrm{~N} 20 \mathrm{~F} 47$ & 860.7 & 873.2 & $\mathrm{~N} 25 \mathrm{~F} 22$ & 1006.16 & 1014 & N25F 47 & 948.72 & 979.84 \\
\hline N20F23 & 743.8 & 743.8 & N20F48 & 910.55 & 910.55 & N25F23 & 933.92 & 933.92 & N25F 48 & 1058 & 1082.72 \\
\hline N2OF24 & 640.7 & 649.3 & N20F49 & 900.35 & 900.35 & N25F24 & 991.76 & 991.76 & $\mathrm{~N} 25 \mathrm{~F} 49$ & 951.84 & 958.4 \\
\hline N20F25 & 802.25 & 876.2 & N20F50 & 903.35 & 910.9 & N25F 25 & 1034.84 & 1044.8 & $\mathrm{~N} 25 \mathrm{~F} 50$ & 898.56 & 909.32 \\
\hline
\end{tabular}




\begin{tabular}{|c|c|c|c|c|c|c|c|c|c|c|c|}
\hline$n=30$ & Optimal & BPNN & $n=20$ & Optimal & BPNN & $n=35$ & Optimal & BPNN & $n=25$ & Optimal & BPNN \\
\hline N30F1 & 1221.53 & 1234.8 & N30F26 & 1216.9 & 1228.13 & N35F 1 & 1455.86 & 1463.54 & N35F 26 & 1547.09 & 1551.91 \\
\hline N30F2 & 1087.5 & 1092.3 & N3OF 27 & 1230.47 & 1234.4 & N35F2 & 1369.2 & 1377.2 & N35F27 & 1390.77 & 1390.77 \\
\hline N30F 3 & 1045.73 & 1174.57 & N30F 28 & 1264.03 & 1266.67 & N35F3 & 1530.29 & 1575.51 & N35F28 & 1466.71 & 1480.31 \\
\hline N30F4 & 1127.37 & 1127,37 & N30F 29 & 1099 & 1099 & N35F 4 & 1302 & 1306.31 & N35F29 & 1062.49 & 1070.43 \\
\hline N30F5 & 1251.67 & 1270.87 & N30F30 & 1189.37 & 1192.7 & N35F5 & 1537.71 & 1557.34 & N35F30 & 1507.97 & 1507.97 \\
\hline N30F6 & 1258.93 & 1334.33 & N3OF31 & 1128.33 & 1128.4 & N35F6 & 1492.11 & 1528.63 & N35F31 & 1452.63 & 1464.26 \\
\hline N30F7 & 1058.77 & 1062.97 & N3OF 32 & 1166.37 & 1172.33 & N35F7 & 1545.17 & 1545.17 & N35F32 & 1129.31 & 1135.46 \\
\hline N30F8 & 1289.8 & 1305.67 & N30F33 & 1127.9 & 1149.87 & N35F8 & 1449.03 & 1462.89 & N35F33 & 1043.17 & 1049.11 \\
\hline N30F9 & 1292.3 & 1294.5 & N30F34 & 1277.67 & 1277.67 & N35F9 & 1393.49 & 1430.4 & N35F34 & 1482.43 & 1521.03 \\
\hline N30F 10 & 970.67 & 970.67 & N30F35 & 1168.63 & 1197.93 & N35F 10 & 1431.09 & 1460.29 & N35F35 & 1337.23 & 1363.14 \\
\hline N30F11 & 1132.1 & 1168.67 & N30F36 & 1337.2 & 1349.87 & N35F 11 & 1491.49 & 1512.31 & N35F 36 & 1567 & 1570.06 \\
\hline N30F12 & 1165.13 & 1176.07 & N30F37 & 1205.57 & 1205.57 & N35F 12 & 1273.09 & 1308.4 & N35F37 & 1184.31 & 1199.46 \\
\hline N30F13 & 1130 & 1148.23 & N30F38 & 1147.43 & 1192.17 & N35F 13 & 1497.54 & 1527.37 & N35F38 & 1353.23 & 1.353 .23 \\
\hline N30F 14 & 1184.73 & 1206 & N30F39 & 1262.7 & 1266.5 & N35F14 & 1472.49 & 1481.06 & N35F39 & 1381.89 & 1455.71 \\
\hline N30F 15 & 1116.33 & 1116.8 & N30F 40 & 1194.5 & 1195.83 & N35F 15 & 1318.34 & 1325.09 & $\mathrm{~N} 35 \mathrm{~F} 40$ & 1214.06 & 1260 \\
\hline N30F 16 & 1100 & 1110.5 & N30F 41 & 1306.77 & 1342 & N35F 16 & 1329 & 1365.71 & N35F 41 & 1483.97 & 1517.6 \\
\hline N30F 17 & 1219.7 & 1250.63 & N30F 42 & 1189.57 & 1229.03 & N35F 17 & 1367.89 & 1373.83 & $\mathrm{~N} 35 \mathrm{~F} 42$ & 1303.6 & 1360.29 \\
\hline N30F 18 & 1362.57 & 1368.73 & N30F 43 & 1239.2 & 1251.37 & N35F 18 & 1298.31 & 1348.09 & N35F 43 & 1136.23 & 1138.4 \\
\hline N30F 19 & 1270.97 & 1328.4 & N30F 44 & 1065.47 & 1065.47 & N35F 19 & 1382.11 & 1384.29 & N35F44 & 1443.6 & 1459.37 \\
\hline N30F 20 & 1343.87 & 1350.87 & N30F 45 & 1292.53 & 1316.83 & N35F20 & 1421.09 & 1540.4 & N35F 45 & 1334.06 & 1390.03 \\
\hline N30F21 & 1113.03 & 1113.03 & N30F 46 & 1141.07 & 1180.23 & N35F21 & 1209.46 & 1209.46 & N35F46 & 1414.83 & 1422.31 \\
\hline N30F22 & 1306.07 & 1306.17 & N30F47 & 1089.97 & 1111.5 & N35F22 & 1241.66 & 1286.06 & N35F 47 & 1149.26 & 1159.4 \\
\hline N30F23 & 1229.43 & 1235.97 & N30F48 & 1236.2 & 1288.9 & N35F23 & 1372.74 & 1391.91 & N35F 48 & 1262.11 & 1305.97 \\
\hline N30F24 & 1374.87 & 1388.67 & N30F49 & 1148.43 & 1161.77 & N35F24 & 1447.29 & 1456.03 & N35F49 & 1400.86 & 1469.4 \\
\hline N30F25 & 932.17 & 938.43 & N30F50 & 1076.3 & 1076.3 & N35F 25 & 1320.83 & 1320.83 & N35F 50 & 1471.14 & 1489.69 \\
\hline
\end{tabular}




\begin{tabular}{|c|c|c|c|c|c|c|c|c|c|c|c|}
\hline$n=40$ & Optimal & BPNN & $n=40$ & Optimal & BPNN & $n=45$ & Optimal & BPNN & $n=45$ & Optimal & BPNN \\
\hline N40F1 & 1497.88 & 1529.03 & N40F26 & 1535.4 & 1543.82 & N45F1 & 2025.58 & 2053.73 & N45F 26 & 1719.53 & 1741.91 \\
\hline N40F2 & 1441.12 & 1457.12 & N40F27 & 1664.8 & 1697.7 & $N 45 F 2$ & 1745.09 & 1771.33 & N45F27 & 1779.11 & 1792.64 \\
\hline N40F3 & 1579.18 & 1579.18 & N40F28 & 1685.53 & 1699 & N45F3 & 1638.73 & 1642.69 & N45F28 & 1530.4 & 1540.64 \\
\hline N4OF4 & 1633.62 & 1647.25 & N40F29 & 1578.85 & 1628.7 & N45F 4 & 1464.33 & 1464.33 & N45F29 & 1827.78 & 1827.78 \\
\hline N40F5 & 1419.3 & 1505.28 & N40F30 & 1604.07 & 1776.43 & N45F5 & 1727.36 & 1729.22 & N45F30 & 1699.36 & 1729.6 \\
\hline N40F6 & 1487.9 & 1603.5 & N40F31 & 1629.6 & 1629.6 & N45F6 & 1826.71 & 1849.04 & N45F31 & 1607.87 & 1677.02 \\
\hline N40F7 & 1062.43 & 1062.43 & N40F32 & 1512.62 & 1547.32 & N45F7 & 1508.8 & 1527.11 & N45F32 & 1852 & 1875.42 \\
\hline N40F8 & 1210.72 & 1248.15 & N40F33 & 1638.45 & 1719.32 & N45F 8 & 1914.78 & 1947.49 & N45F33 & 1839.73 & 1845.76 \\
\hline N40F9 & 1367.9 & 1380.8 & N40F34 & 1504.07 & 1506.7 & N45F9 & 1623.09 & 1624.98 & N45F34 & 1605.84 & 1651.53 \\
\hline N4OF 10 & 1216 & 1216 & N40F35 & 1638.35 & 1716.32 & N45F 10 & 1371.76 & 1378.56 & N45F35 & 1986.38 & 1986.38 \\
\hline N40F11 & 1479.68 & 1481.12 & N40F36 & 1551.5 & 1603.8 & N45F 11 & 1661.07 & 1721.38 & N45F36 & 1590.53 & 1594.31 \\
\hline N40F 12 & 1224.03 & 1271.3 & N40F37 & 1729.65 & 1763.22 & N45F 12 & 1358.78 & 1424.36 & N45F37 & 1495.73 & 1530.24 \\
\hline N4OF 13 & 1279.03 & 1281.3 & N40F38 & 1211.12 & 1223.6 & N45F13 & 1639.4 & 1644 & N45F38 & 1661.24 & 1669.29 \\
\hline N40F 14 & 1435.18 & 1448.35 & N40F39 & 1407.9 & 1434.53 & N45F 14 & 1732.96 & 1757.8 & N45F39 & 1689.51 & 1689.73 \\
\hline N40F 15 & 1117.43 & 1132.47 & N40F 40 & 1058.75 & 1065.72 & N45F 15 & 1862.67 & 1892.04 & N45F40 & 1979.67 & 2028.33 \\
\hline N40F 16 & 1693.2 & 1716.45 & N40F41 & 1199.78 & 1202.78 & N45F 16 & 1758.11 & 1799.38 & N45F41 & 1611.31 & 1642.44 \\
\hline N4OF 17 & 1410.09 & 1457.03 & N40F 42 & 1579.95 & 1590.5 & N45F 17 & 1655.44 & 1697.91 & N45F42 & 1509.69 & 1571.18 \\
\hline N4OF 18 & 1475.79 & 1502.85 & N40F 43 & 1632.35 & 1668.97 & N45F18 & 1370.36 & 1376.64 & $\mathrm{~N} 45 \mathrm{~F} 43$ & 1740.67 & 1788.6 \\
\hline N4OF19 & 1649.03 & 1671.22 & N40F 44 & 1633 & 1649.25 & N45F19 & 1835.53 & 1849.73 & N45F44 & 1566.33 & 1615.44 \\
\hline N40F20 & 1554 & 1585.2 & N40F45 & 1540.35 & 1540.35 & N45F2O & 1605.04 & 1617.78 & N45F45 & 1821.58 & 1831.22 \\
\hline N40F21 & 1509.62 & 1532.5 & N4OF46 & 1766.22 & 1821.53 & N45F21 & 1823.44 & 1843.67 & $N 45 F 46$ & 1491.69 & 1527.8 \\
\hline N4OF22 & 1198.72 & 1261.85 & N40F 47 & 1552.55 & 1554.35 & N45F22 & 1696.71 & 1712.38 & N45F47 & 1872.42 & 1877.51 \\
\hline N4OF23 & 1567.7 & 1568.57 & N40F48 & 1726.55 & 1726.82 & N45F23 & 1920.98 & 1956.64 & N45F48 & 1867.93 & 1922.84 \\
\hline N40F24 & 1516.28 & 1540.7 & N40F49 & 1214.72 & 1216.7 & N45F24 & 1799.69 & 1809.87 & N45F49 & 1875.16 & 1895.2 \\
\hline N40F 25 & 1340.5 & 1357.8 & N40F50 & 1410.88 & 1410.88 & N45F25 & 1384.47 & 1384.47 & N45F50 & 1725.31 & 1744.11 \\
\hline
\end{tabular}




\begin{tabular}{|c|c|c|c|c|c|c|c|c|c|c|c|}
\hline$n=55$ & Optimal & BPNN & $n=55$ & Optimal & BPNN & $n=60$ & Optimal & BPNN & $n=60$ & Optimal & BPNN \\
\hline N55F1 & 2097.36 & 2130.89 & N55F26 & 1942.71 & 2025.45 & N60F1 & 2086.93 & 2126.2 & N60F26 & 2242.73 & 2244.63 \\
\hline N55F2 & 2272.09 & 2288.35 & N55F27 & 2272.96 & 2352.35 & N60F2 & 2236.13 & 2238.17 & N60F 27 & 2101.72 & 2116.62 \\
\hline N55F3 & 1949.64 & 1949.64 & N55F28 & 2063.78 & 2113.98 & N60F3 & 2400.28 & 2400.28 & N60F 28 & 1724.43 & 1760.8 \\
\hline N55F4 & 2103.84 & 2115.02 & N55F29 & 2141.93 & 2240.38 & N60F4 & 2147.48 & 2150.03 & N60F 29 & 2368.58 & 2380 \\
\hline N55F5 & 2154.02 & 2159.02 & N55F30 & 2137.78 & 2137.78 & N60F5 & 2054.78 & 2059.48 & N60F 30 & 2507.32 & 2555.77 \\
\hline N55F6 & 1980.65 & 1980.65 & N55F31 & 2201.27 & 2212.6 & N60F6 & 2465.25 & 2471.83 & N60F 31 & 2169.57 & 2239.82 \\
\hline N55F7 & 1970.53 & 2040.47 & N55F32 & 1970.87 & 1990.71 & N60F7 & 1922.17 & 1922.17 & N60F32 & 2318.53 & 2429.42 \\
\hline N55F8 & 1766.71 & 1766.71 & N55F33 & 2272.6 & 2279.85 & N60F8 & 2204.28 & 2204.28 & N60F33 & 2386.35 & 2477.6 \\
\hline N55F9 & 2062.84 & 2070.75 & N55F34 & 2114.49 & 2117.05 & N60F9 & 1910.17 & 2031.88 & N60F 34 & 1885.15 & 1902.03 \\
\hline N55F10 & 1892.93 & 1892.93 & N55F35 & 1920.04 & 1924.13 & N60F 10 & 2463.3 & 2475.5 & N60F 35 & 2260.57 & 2262.43 \\
\hline N55F11 & 2187.91 & 2211.98 & N55F36 & 2281.33 & 2281.33 & N60F 11 & 1925.38 & 1925.38 & N60F36 & 2011 & 2012.07 \\
\hline N55F12 & 2028.45 & 2028.45 & N55F37 & 2046.85 & 2087.64 & N60F 12 & 2320.52 & 2320.52 & N60F 37 & 2163.82 & 2164.8 \\
\hline N55F13 & 2375.44 & 2475.16 & N55F38 & 1945.4 & 1947.93 & N60F13 & 2201.5 & 2312.03 & N60F38 & 2312.53 & 2474.52 \\
\hline N55F 14 & 1936.8 & 2016.31 & N55F39 & 2085.22 & 2086.2 & N60F 14 & 2247.55 & 2247.55 & N60F39 & 2197.25 & 2199.28 \\
\hline N55F15 & 2334.51 & 2356.69 & N55F 40 & 1751.87 & 1773.6 & N60F 15 & 1718.22 & 1737.62 & N60F 40 & 2373.08 & 2386.22 \\
\hline N55F16 & 2046.67 & 2111.53 & N55F41 & 1959.18 & 1960.85 & N60F16 & 2250.02 & 2258.02 & N60F 41 & 2428.48 & 2438.78 \\
\hline N55F 17 & 1959.47 & 1972.11 & N55F42 & 1940.84 & 1972.98 & N60F 17 & 2278.28 & 2279.2 & N60F 42 & 2256 & 2335.1 \\
\hline N55F 18 & 2180.58 & 2180.58 & N55F43 & 1954.76 & 1970.87 & N60F 18 & 2434.25 & 2502.22 & N60F 43 & 2361.22 & 2363.17 \\
\hline N55F19 & 2227.65 & 2283.24 & N55F44 & 2290.8 & 2294.85 & N60F 19 & 2136.57 & 2147.62 & N60F 44 & 2225 & 2227.68 \\
\hline N55F20 & 1934.2 & 1967.02 & N55F45 & 2104.71 & 2104.71 & N60F20 & 1966.78 & 1966.78 & N60F 45 & 2294.48 & 2296.52 \\
\hline N55F21 & 1950.02 & 1954.8 & N55F 46 & 2216.45 & 2216.45 & N60F 21 & 2397.02 & 2397.43 & N60F 46 & 1973.8 & 1974.53 \\
\hline N55F22 & 1924.73 & 1925 & N55F47 & 2059.38 & 2060.58 & N60F 22 & 2278.28 & 2344.5 & N60F47 & 2406.68 & 2416.08 \\
\hline N55F23 & 2252.87 & 2244.16 & N55F48 & 2321.11 & 2351.49 & N60F23 & 1921.82 & 1952.1 & N60F 48 & 2357 & 2378.88 \\
\hline N55F24 & 1857 & 1857.55 & N55F49 & 1900.27 & 1900.27 & N60F24 & 2141.77 & 2143.7 & N60F49 & 2274.28 & 2309.03 \\
\hline N55F25 & 2358.55 & 2379.04 & N55F50 & 2083.02 & 2083.02 & N60F 25 & 2383.47 & 2401.17 & N60F50 & 2272.98 & 2484.62 \\
\hline
\end{tabular}




\begin{tabular}{|c|c|c|c|c|c|c|c|c|c|c|c|}
\hline$n=75$ & Optimal & BPNN & $n=75$ & Optimal & BPNN & $n=85$ & Optimal & BPNN & $n=85$ & Optimal & BPNN \\
\hline $\mathrm{N} 75 \mathrm{~F} 1$ & 2851.16 & 2991.17 & N75F26 & 2766.87 & 2884.39 & N85F1 & 3047.98 & 3089.78 & N85F26 & 3317.27 & 3348.74 \\
\hline N75F2 & 2888.11 & 2901.88 & N75F27 & 3013.07 & 3061.61 & N85F2 & 2961.82 & 3006.07 & N85F27 & 3097.36 & 3110.96 \\
\hline N75F3 & 2748.17 & 2766.13 & N75F28 & 2570.76 & 2588.56 & N85F3 & 3330.11 & 3402.32 & N85F28 & 2798.93 & 2803.38 \\
\hline N75F4 & 2511.31 & 2541.51 & N75F29 & 3018.77 & 3027.73 & N85F 4 & 3181.13 & 3229.29 & N85F29 & 2765.48 & 2766.25 \\
\hline N75F5 & 2890.39 & 2998.4 & N75F30 & 1921.05 & 1924.57 & N85F5 & 2815.17 & 2828.29 & N85F30 & 2939.81 & 3114.24 \\
\hline N75F6 & 2969.59 & 3030.85 & N75F31 & 2699.79 & 2719.08 & N85F6 & 3370.56 & 3370.56 & N85F31 & 3002.96 & 3115.6 \\
\hline N75F7 & 2138.59 & 2141.04 & N75F 32 & 2848.44 & 2848.65 & N85F7 & 2951.12 & 2962.91 & N85F32 & 3118.47 & 3283.88 \\
\hline N75F8 & 2887 & 2887 & N75F 33 & 2565.6 & 2620.24 & N85F8 & 3414.59 & 3414.79 & N85F33 & 2959.16 & 2979.38 \\
\hline N75F9 & 2931.55 & 2974.96 & N75F34 & 2694.89 & 2694.89 & N85F9 & 3014.12 & 3019.28 & N85F34 & 2877.71 & 2887.12 \\
\hline N75F 10 & 2832.8 & 2854.69 & N75F35 & 2804.84 & 2804.84 & N85F 10 & 3445.29 & 3507.76 & N85F35 & 3084.39 & 3107.14 \\
\hline N75F 11 & 2987.37 & 2993.48 & N75F36 & 2678.88 & 2686.32 & N85F 11 & 2862.02 & 2872.59 & N85F36 & 3203.53 & 3241.13 \\
\hline N75F 12 & 2734.88 & 2735.2 & N75F37 & 2617.49 & 2695.67 & N85F 12 & 3295.66 & 3295.66 & N85F37 & 3169.92 & 3172.2 \\
\hline N75F 13 & 2700.99 & 2730.56 & N75F38 & 2482.28 & 2584.56 & N85F 13 & 3244.08 & 3305.55 & N85F38 & 3077.67 & 3095.65 \\
\hline N75F 14 & 2603.77 & 2612 & N75F39 & 3022.65 & 3022.65 & N85F 14 & 2891.46 & 3021.85 & N85F39 & 319 & 3250.72 \\
\hline N75F 15 & 2494.01 & 2528 & N75F 40 & 2823.95 & 2828.83 & N85F 15 & 3142.85 & 3186.96 & N85F 40 & 2162.98 & 2214.61 \\
\hline N75F16 & 2908.52 & 3025,49 & N75F 41 & 2642.83 & 2712.24 & N85F16 & 3305.38 & 3357.52 & N85F 41 & 3208.14 & 3251.32 \\
\hline N75F17 & 2986 & 3003.8 & N75F42 & 2881.43 & 2883.49 & N85F 17 & 2817.04 & 2884.08 & N85F 42 & 3119.93 & 3188.22 \\
\hline N75F 18 & 2805.21 & 2805.21 & $N 75 F 43$ & 2592.92 & 2602.95 & N85F18 & 3112.8 & 3182.36 & N85F 43 & 3175.6 & 3177.66 \\
\hline N75F19 & 2698.67 & 2698.67 & N75F44 & 2848.63 & 2882.16 & N85F 19 & 3078.24 & 3080.74 & N85F 44 & 3428.38 & 3432.4 \\
\hline N75F20 & 2769.4 & 2793.55 & N75F45 & 2391.95 & 2396.99 & N85F20 & 3182.84 & 3313.68 & N85F 45 & 2586.18 & 2606.24 \\
\hline N75F21 & 3021.55 & 3129.35 & N75F46 & 2172.41 & 2175.57 & N85F21 & 2965.42 & 2977.95 & N85F 46 & 2878.28 & 2878.28 \\
\hline N75F22 & 2088.49 & 2088.49 & N75F47 & 2736.45 & 2736.45 & N85F22 & 3296.13 & 3309.12 & N85F 47 & 3301.33 & 3301.33 \\
\hline N75F23 & 3021.55 & 2880.2 & N75F48 & 2582.29 & 2583.37 & N85F23 & 3155.89 & 3228.99 & N85F48 & 3093.54 & 3093.54 \\
\hline N75F24 & 2932.59 & 2956.39 & $\mathrm{~N} 75 \mathrm{~F} 49$ & 2949.23 & 3048.27 & N85F24 & 2856.11 & 2872.75 & N85F49 & 2417.16 & 2433.12 \\
\hline N75F25 & 2834.67 & 2954.33 & N75F50 & 2722.45 & 2722.89 & N85F25 & 3075.45 & 3118.69 & N85F50 & 3149.61 & 3215.18 \\
\hline
\end{tabular}




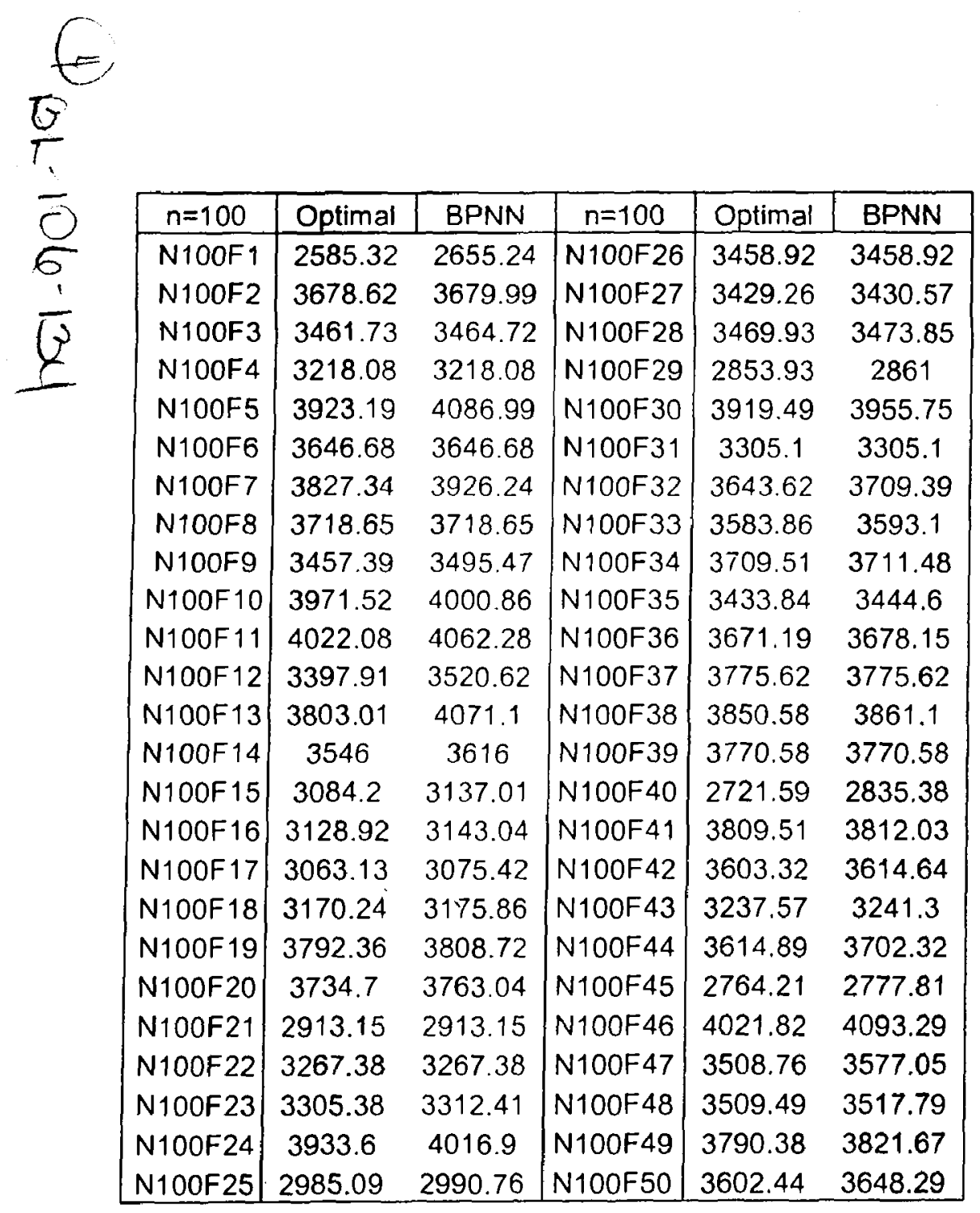

